\title{
Identification of Conserved Aquilegia Coerulea MicroRNAs and Their Targets
}

\section{Citation}

Puzey, Joshua Robert, and Elena M. Kramer. 2009. Identification of conserved Aquilegia coerulea microRNAs and their targets. Gene 448(1): 46-56.

\section{Published Version}

doi:10.1016/j.gene.2009.08.005

\section{Permanent link}

http://nrs.harvard.edu/urn-3:HUL.InstRepos:8519268

\section{Terms of Use}

This article was downloaded from Harvard University's DASH repository, and is made available under the terms and conditions applicable to Open Access Policy Articles, as set forth at http:// nrs.harvard.edu/urn-3:HUL.InstRepos:dash.current.terms-of-use\#OAP

\section{Share Your Story}

The Harvard community has made this article openly available.

Please share how this access benefits you. Submit a story.

\section{Accessibility}




\section{Elsevier Editorial System(tm) for Gene Manuscript Draft}

Manuscript Number: GENE-D-09-00303R1

Title: Identification of conserved Aquilegia coerulea microRNAs and their targets.

Article Type: Research Paper

Keywords: Aquilegia; microRNA; evolution; angiosperm

Corresponding Author: Dr. Elena M. Kramer,

Corresponding Author's Institution: Harvard University

First Author: Joshua R Puzey

Order of Authors: Joshua R Puzey; Elena M. Kramer

Manuscript Region of Origin: UNITED STATES

Abstract: Aquilegia is an emerging model organism that is phylogenetically intermediate between the core eudicot and monocot models Arabidopsis and Oryza. In this study, we have used a comparative genomics approach to identify 45 Aquilegia microRNAs that comprise 20 separate plant microRNA families. We have predicted 85 targets of these newly identified Aquilegia microRNAs, including transcription factors and loci involved in metabolism, stress responses, transport, and auxin signaling. microRNA families from 16 plant species and the newly identified microRNAs from Aquilegia were analyzed in a phylogenetic context revealing 40 distantly conserved microRNA families. In addition to these highly conserved plant microRNA families, several families with disjointed phylogenetic distribution were identified. This study provides a phylogenetically important dataset for plant microRNA evolution studies. The current study is the first to identify miRNAs in a lower eudicot in which comprehensive genomic resources are becoming available. 


\title{
Identification of conserved Aquilegia coerulea microRNAs and their targets
}

\author{
Joshua R. Puzey and Elena M. Kramer*
}

Dept. of Organismic and Evolutionary Biology, Harvard University, Cambridge MA 02138

*Author for Correspondence: 16 Divinity Ave, Cambridge MA 02138;

ekramer@oeb.harvard.edu; phone: 617-496-3460, fax: 617-496-5854 


\begin{abstract}
Aquilegia is an emerging model organism that is phylogenetically intermediate between the core eudicot and monocot models, Arabidopsis and Oryza. In this study, we have used a comparative genomics approach to identify 45 Aquilegia microRNAs that comprise 20 separate plant microRNA families. We have predicted 85 targets of these newly identified Aquilegia microRNAs including transcription factors and loci involved in metabolism, stress responses, transport, and auxin signaling. microRNA families from 16 plant species and the newly identified microRNAs from Aquilegia were analyzed in a phylogenetic context revealing 40 distantly conserved microRNA families. In addition to these highly conserved plant microRNA families, several families with disjointed phylogenetic distribution were identified. This study provides a phylogenetically important dataset for plant microRNA evolution studies. The current study is the first to identify miRNAs in a lower eudicot in which comprehensive genomic resources are becoming available.
\end{abstract}

Keywords: Aquilegia, microRNA, evolution, angiosperm 


\section{Introduction}

microRNAs (miRNAs) are a set of small ( 22 nt) single-stranded non-coding RNAs in plants and animals that play an important role in regulating mRNA targets through cleavage and/or translational repression in a sequence specific manner (Chen, 2004; Jones-Rhoades et al., 2006). In plants, miRNAs have diverse roles including the regulation of leaf development (Palatnik et al., 2003), floral development (Cartolano et al., 2007), phase change (Aukerman and Sakai, 2003; Lauter et al., 2005), male and female reproductive development (Wu et al., 2006), root development (Boualem et al., 2008), and disease and environmental stress response (Shukla et al., 2008; Zhang et al., 2008b; Ding et al., 2009).

Evolutionary studies of plant miRNAs are currently limited by the large phylogenetic distances between plant miRNA datasets (Fig. 1). The four best-annotated plant model species in which significant miRNA datasets have been determined include the core eudicot, Arabidopsis thaliana (A. thaliana), the monocot, Oryza sativa (O. sativa), the lycopod, Selaginella moellendorffii (S. moellendorffii), and the bryophyte Physcomitrella patens (P. patens) (Fig. 1). The eudicot and monocot lineages are estimated to have diverged from one another approximately $140 \mathrm{Myr}$ ago, while lineage containing Aquilegia diverged from other eudicots approximately 100 Myr ago (Fig. 1) (Chaw et al., 2004; Sanderson et al., 2004; Moore et al., 2007). The Aquilegia miRNA dataset therefore helps to break up the large phylogenetic distance that separates major flowering plant lineages, particularly the monocots and dicots.

The goal of this study is to annotate the miRNA profile of Aquilegia. Aquilegia (columbine), a eudicot and a member of the Ranunculales, is an emerging model 
organism with a large number of available genetic and genomic tools, including ongoing whole genome sequencing. The phylogenetic position of Aquilegia will provide an important reference point for comparing these core eudicot and monocots models, while allowing us to ask questions about the origin and diversification of major plant miRNA lineages. In addition to having a critical position for deep phylogenetic analyses, Aquilegia has undergone a recent adaptive radiation due to diverse ecological niches (Hodges and Arnold, 1994b; Hodges, 2003; Hodges and Kramer, 2007). This recent adaptive radiation coupled with genomic tools will allow us to elucidate the genetic basis for morphological variation and speciation in the genus.

The evolution and conservation of plant miRNAs has been the subject of significant investigation (Axtell and Bartel, 2005; Zhang et al., 2006a; Axtell and Bowman, 2008). In this study we have identified multiple highly conserved plant miRNAs in Aquilegia as well as miRNA families that have a more disjointed phylogenetic distribution.

\section{Materials and Methods}

\subsection{Aquilegia coerulea database}

We searched for miRNAs among the currently available genomic sequences from Aquilegia coerulea 'Goldsmith'. This is a horticultural inbred line derived from several species, but primarily Aquilegia coerulea. This inbred line is referred to as A. coerulea

for the remainder of this paper. Currently, whole genome sequence consists of 483,253 reads corresponding to an estimated $0.7 \mathrm{x}-1 \mathrm{x}$ genome coverage and is available at the Trace Archives at NCBI under "Aquilegia coerulea - WGS". 


\subsection{Criteria for orthologous miRNA annotation}

Orthologous miRNAs were identified according to the criteria for conserved plant miRNA annotation established by Meyers et al. (2008). These criteria include conservation of the miRNA precursor hairpin and the mature miRNA sequence. The specific criteria for filtering the stem-loop structure and the mature miRNA sequence conservation are as follows: no more than four mismatches between miRNA/miRNA* were allowed; no bulges in miRNA/miRNA* larger than two bases; and four or fewer mismatched sequences were allowed between the $A$. coerulea miRNA and the previously identified miRNA (Meyers et al., 2008).

\subsection{Mature miRNA dataset}

A total of 1023 mature miRNA sequences from Glycine max (G. max) (number of mature sequences $=42)$, Medicago truncatula (M. truncatula) (34), Gossypium hirsutum (G. hirsutum) (13), A. thaliana (97), Brassica rapa (B. rapa) (15), Brassica napus (B. napus) (41), Populus trichocarpa (P. trichocarpa) (181), Vitis viniferia (V. vinifera) (133), Solanum lycopersicum (S. lycopersicum) (24), Triticum aestivum (T. aestivum) (9), O. sativa (159), Sorghum bicolor (S. bicolor) (78), Zea mays (Z. mays) (97), Pinus taeda (P. taeda) (19), Selaginella moellendorffii (S. moellendorffii) (18), and Physcomitrella patens (P. patens) (64) were obtained from mirBASE (Release 13.0) and the recently published S. bicolor genome (Griffiths-Jones, 2004; Griffiths-Jones et al., 2006; Griffiths-Jones et al., 2008; Paterson et al., 2009). Redundant sequences were removed from this dataset. miRNA families from this dataset that were conserved in two or more plant species were searched for in A. coerulea. The non-redundant sequences were then used as the query in a BLASTn search of the A. coerulea genome (see below). 


\subsection{BLASTn of A. coerulea genome}

We performed a BLASTn search of the A. coerulea whole genome sequence using the following parameters: database was set to Aquilgia coerulea-WGS; BLASTn was chosen; 'automatically adjust parameters for short input sequences', which is the default, was deselected to allow us to create our own search profile; the expect threshold was set to 1000 ; the word size was set to 7 ; and all other parameters were left at default. The BLASTn was performed and all sequences with four or fewer mismatches across the entire query sequence were extracted and stored in a database for further analysis. Our BLASTn method identified miRNA binding sites in coding genes in addition to miRNAs. True miRNAs were identified by their indicative secondary hairpin structure (see below) while the remaining sequences were filtered out of our database (Fig. 2). These latter sequences could represent target coding genes but the available genome sequence has yet to be annotated.

\section{5 miRNA hairpin prediction}

All sequences that had four or fewer mismatches with previously identified mature miRNAs were then filtered using their predicted secondary structure (Fig. 2). Mfold, a publicly available online application (http://mfold.bioinfo.rpi.edu), was used to predict the secondary structure of the obtained sequences based on thermodynamic stability (Mathews et al., 1999; Zuker, 2003). The RNA folding application was used and all parameters were left at default. Because the RNA folding application only accepts sequences shorter than $800 \mathrm{nt}$, for sequences longer than $800 \mathrm{nt}$ we performed two steps: (1) the Nucleic Acid Quikfold application available at Mfold (an application capable of accepting longer sequences) was used to predict secondary structure and (2) CLUSTALw 
alignment with mature miRNA sequence was performed. After the Nucleic Acid Quickfold and CLUSTALw alignment, the regions furthest from the predicted hairpin and mature sequence site was trimmed off, shortening the sequence to $800 \mathrm{nt}$. This shortened sequenced was then analyzed in Mfold. The structure with the highest score and lowest free energy was analyzed and the precursor sequence was predicted based on secondary folding structure. The extent of the precursor sequence was predicted by identifying any large loops with little or no nucleotide pairing that followed the end of a region with significant pairing. Secondary structures were then screened for four or fewer mismatches in the miRNA/miRNA* duplex and a folding energy of lower than -15 $\mathrm{kcal} / \mathrm{mold}$. Our initial BLAST identified 240 of sequences of which we have only predicted 45 to be miRNAs.

\subsection{MFE/AMFE/MFEI}

The minimal folding energy (MFE), expressed in $\mathrm{kcal} / \mathrm{mol}$, is a method of calculating the thermodynamic stability of the secondary structure of RNA or DNA (Mathews et al., 1999; Zuker, 2003). The lower the MFE of a molecule, the more stable the secondary structure. Because MFE values are strongly correlated with the length of the sequence we normalized the MFE by calculating the adjusted MFE (AMFE) (Zhang et al. 2008) using the following equation: AMFE=[(MFE/length of RNA sequence) x 100] (Zhang et al., 2006b). Next, the minimal folding free energy index (MFEI) was calculated for the $A$. coerulea miRNA precursors. The MFEI is an index developed by Zhang et al. (2006) and is used as a criterion to differentiate between miRNAs versus other RNA based on MFE, sequence length, and G+C nucleotide composition (Zhang et al., 2006b; Zhang et al., 2008a). The minimal folding free energy index (MFEI) was calculated by the following equation: $\mathrm{MFEI}=[(\mathrm{AMFE}) \times 100] /(\mathrm{G} \%+\mathrm{C} \%)]$ (Zhang et al., 2006b). 


\section{7 miRNA target prediction}

The near-perfect complementarity of plant miRNAs for their targets allows for very accurate prediction of miRNA targets (Rhoades et al., 2002; Jones-Rhoades and Bartel, 2004; Schwab et al., 2005; Schwab et al., 2006). All non-redundant A. coerulea mature miRNA sequences were used as the query in a BLASTn search of the Aquilegia Gene Index database, which consists of 85,039 reads (http://compbio.dfci.harvard.edu/tgi/cgibin/tgi/Blast/index.cgi). The Aquilegia Gene Index (AqGI) was used because opening reading frame (ORF) predictions have been made for these sequences and the A. coerulea whole genome sequence has not yet been assembled. The AqGI is based on a full-length enriched, normalized cDNA library derived from a wide range of tissue collected from Aquilegia formosa X Aquilegia pubescens including shoot meristems, floral apical meristems, flower buds, leaves, and root tissue. The exceptionally low sequence variation between Aquilegia species (Hodges and Arnold, 1994a) allows us to use this database to accurately predict targets for A. coerulea miRNAs.

Targets were identified via BLASTn using the following method: BLASTn expected value was raised to 10,000 ; sequences were filtered based on four or fewer mismatches with the query; and no gaps were allowed at the binding site. The Aquilegia sequences with four or fewer mismatches were then extracted and a BLASTn search was

performed with these sequences against Arabidopsis genome (http://www.arabidopsis.org/Blast/index.jsp). The top hit was selected for each Aquilegia EST and its name, biological process, and molecular function were obtained. We also aligned predicted target ESTs for each miRNA family against one another in order to eliminate redundancies (ESTs that shared greater than 98\% sequence identity, usually due to separate annotations of alternative splicing products of the same locus). 
For miRNA families not yet identified in A. coerulea, potential targets were identified using target BLASTn method described above, but with non-redundant mature miRNA sequences from other species as the query. Although identification of a conserved miRNA binding site in a target is not sufficient evidence for definitively establishing a miRNA family's presence in the genome, conservation of targets provides supporting evidence for their presence. Targets were only predicted for miRNA families that are conserved across two deeply divergent land plant lineages (eg. moncots and dicots or dicots and bryophytes). miRNA families that met these criteria were: 162, 164, 390, 393, 394, 397, and 783 (see section 3.5).

\section{Results and Discussion}

\subsection{Identification of Aquilegia coerulea miRNAs}

A total of 45 miRNAs from 20 miRNA families were identified in A. coerulea (Table 1 , Supplementary Material (Supp.) Fig. 3A). Family size ranged from one to seven members with seven miRNAs belonging to miR477; six to miR171; five to miR166; three to miR169 and miR482; two to miR156, miR160, miR172, miR395, miR396, and miR398; and one miRNA identified for the remaining families (Supp. Fig. 3A). It is important to note that aqc-miR530 has a three nucleotide bulge on the miRNA* side of the hairpin but was still annotated as a miRNA as it has a MFEI value of 0.88 (see below), indicating that this is likely to be a true miRNA. Similarly, all miRNA annotation criteria as described in Meyers et al. (2008), except for a three nucleotide bulge in the miRNA*, were met for aqc-miR168. aqc-miR168 was included in our miRNA dataset since it has a low folding energy of $-40.45 \mathrm{kcal} / \mathrm{mol}$, in spite of the three 
nucleotide bulge, indicating it has a relatively stable secondary structure.

The majority of mature miRNAs $(66.7 \%)$ were $21 \mathrm{nt}$ in length, with $24.4 \%$ and $8.9 \%$ were $20 \mathrm{nt}$ and $22 \mathrm{nt}$ in length, respectively (Table 1). Nucleotide composition of the mature miRNAs was analyzed. Cytosine is the dominant nucleotide totaling $30.2 \%$ of the mature miRNA nucleotide composition; uracil is the next most prevalent comprising $26.7 \%$ of the mature sequence, followed by guanine (21.7\%), and adenine (21.4\%) (Fig. 3).

It has been previously reported that the strong bias of uracil in the first 5, nucleotide position is due to its important role in the recognition of the miRNA by ARGONAUTE1 (Mi et al., 2008; Montgomery et al., 2008; Takeda et al., 2008; Zhang et al., 2008a). Consistent with this, we found in A. coerulea that uracil (71.1\%) was the dominant nucleotide at the first position of the 5' end of the mature A. coerulea miRNAs (Fig. 3). Conclusions on the importance of position-specific nucleotide preference at sites other than the first nucleotide of the miRNA have varied. Zhang et al. (2008) reported $61 \%$ cytosine preference at position 19 in soybean and suggested that this may be important for RISC or Dicer cleavage sites on the miRNA precursor while Mi et al. (2008) reported that, aside from the first position, no other position-specific nucleotide preference could be determined. Similar to Zhang et al (2008), in A. coerulea, we observed a strong preference, $51.1 \%, 55.6 \%$, and $51.5 \%$, cytosine at positions 18,19 , and 21, respectively (Fig. 3). We agree with Zhang et al. that the most likely explanation for this similarity across otherwise divergent miRNA families is some kind of biochemical constraint related to miRNA processing.

\subsection{Precursor Analysis}


The length of the predicted Aquilegia miRNA precursors varies from 68 to $180 \mathrm{nt}$ with an average precursor length of $106 \pm 32 \mathrm{nt}$ (Table 2). It is important to note that these precursors are predictions based on their secondary folding structure (see Methods). All predicted $A$. coerulea miRNA hairpins are available in the supplementary information but several representative $A$. coerulea miRNA hairpin structures are shown in (Fig. 4). The nucleotide composition of the miRNA precursor sequences in order of abundance is, uracil $(31.4 \pm 3.8 \%)$, adenine $(25.6 \pm 4.4 \%)$, guanine $(22.9 \pm 3.7 \%)$, and cytosine $(20.1 \pm$ $3.0 \%$ ) (Table 2). Previous calculations of nucleotide composition of miRNA-precursors have reported similar values (Zhang et al., 2008a).

The MFE for predicted $A$. coerulea miRNA precursors averaged $-46 \pm 14.7$ ( $\mathrm{kcal} / \mathrm{mol}$ ) and ranged from $-81.8 \mathrm{kcal} / \mathrm{mol}$ to $-18.8 \mathrm{kcal} / \mathrm{mol}$ (Table 2$)$. The average AMFE of the A. coerulea miRNA precursors is $-44.1 \pm 7.5(\mathrm{kcal} / \mathrm{mol})$ (Table 2$)$. The MFEI of A. coerulea miRNA precursors was also analyzed and they scored an average of $1.03 \pm 0.18$, with the lowest score being 0.60 and the highest score being 1.48 (Table 2). Zhang et al. (2006) showed that a MFEI value greater than or equal to 0.85 is a strong indication of an actual miRNA. Of our identified A. coreulea miRNA precursors, 38 (84.4\%) had a MFEI greater or equal to 0.85 and of the remaining seven, two (4.4\%) had a MFEI between 0.6 and 0.7 , three $(6.6 \%)$ between 0.71 and 0.8 , and two (4.4\%) between 0.81 and 0.84 . While a MFEI value above 0.85 is highly indicative of an actual miRNA, lower values do not rule out a sequence as a true miRNA (Zhang et al., 2006b; Zhang et al., 2007).

\subsection{Target Annotation}

We have predicted a total of 85 miRNA targets for $A$. coerulea. Because the A. coerulea 
genome is not yet annotated, these predicted target sequences were compared to the $A$. thaliana database in order to obtain information on potential gene functions. The $A$. thaliana loci with the lowest e-value were selected, corresponding to a total of 71 distinct A. thaliana loci. In several cases, multiple Aquilegia targets recovered the same top A. thaliana hit, resulting in fewer A. thaliana loci (71) than predicted Aquilegia targets (85). This difference could be due to intervening gene duplications between A. thaliana and Aquilegia as well as the inexactitude of BLAST searches in assigning orthology. It does not appear that identification of targeted transposable elements is a factor in the multiple redundant BLAST hits as only one such target was characterized. AqGI TC and EST numbers representing redundant sequences (those with greater than $98 \%$ similarity) were removed from Table 3 (the numbers for every recovered AqGI TC and EST are present in supplementary table 2). In agreement with the fact that miRNAs are important developmental regulators (Aukerman and Sakai, 2003; Palatnik et al., 2003; Chen, 2004), $28.2 \%$ of the predicted targets are inferred to encode transcription factors (Table 3 ). Of the remaining targets, $26.8 \%$ are predicted to be involved in metabolism; $14.1 \%$, stress response; $4.2 \%$, transport; $4.2 \%$, kinases; $2.9 \%$, photo processes; $2.9 \%$, auxin signaling; 1.4\%, RNA binding; and 1.4\%, protein binding (Table 3).

\subsection{Related miRNA families}

\section{$\operatorname{miR156/529}$}

Based on our sequence analysis, aqc-miR156 and aqc-miR529 appear to be related (Fig. 5A,B), which has also been suggested for these families in P. patens (Axtell et al., 2007). The miR156 family is present in the majority of land plants while miR529 is more narrowly distributed and has only been described in $P$. patens, $S$. bicolor, and $O$. sativa 
(as determined from miRBASE and recently published S. bicolor genome) (Fig. 6) (Paterson et al., 2009). Barakat et al. (2007) computationally predicted a miR529 locus in both A. thaliana and P. trichocarpa, although these sequences are not present in miRBASE. To our knowledge, no other reports have identified miR529 in A. thaliana or $P$. trichocarpa, even though small-RNA deep sequencing has been conducted in both these species (Barakat et al., 2007; Fahlgren et al., 2007). A. thaliana and P. trichocarpa have well annotated genomes and it is unlikely, given the current emphasis on miRNA identification, that miR529's presence would have been missed in these model systems. Given these facts, we did not include $A$. thaliana or $P$. trichocarpa miR529 in our summary of the phylogenetic distribution of miRNAs (Fig. 6).

One of our identified sequences, 202185620929, could possibly encode both miR156 and miR529 (Fig. 5A, B). Our prediction that it encodes a miR529 family miRNA is based on the $C C C$ repeat at the $3^{\prime}$ end of the mature miRNA, which is conserved in all P. patens miR529 members (Axtell et al., 2007) (Fig. 5B). Two other sequences (2185892723 and 2185518132$)$ were placed in the miR156 family since neither of these two sequences have a $C C C$ repeat at the 3' end (Fig. 5B). In addition, aqc-miR156 sequences have $T$ at the $7^{\text {th }}$ position as opposed to 202185620929 , which has a $G$ at the $7^{\text {th }}$ position (Fig. 5B).

Overlapping and specific targets were predicted for each of these miRNAs. aqcmiR156 is predicted to regulate five SQUAMOSA PROMOTER BINDING PROTEINLike (SBP) genes and one homolog of Growth Regulating Factor 2 (Table 3). miR156 regulation of the above targets has been shown to play important developmental roles in other plants species, including regulating plastochron length (Wang et al., 2008), organ 
size (Wang et al., 2008), cell number (Usami et al., 2009), and phase change (Gandikota et al., 2007). Guo et al. (2008) reported that the miR156 binding site is highly conserved in land plant SBP genes so our Aquilegia predictions are consistent with these obervations (Guo et al., 2008). aqc-miR529 shares four predicted SBP targets with aqcmiR156 but, in addition, has five specific predicted targets, including two involved in stress response, one involved in metabolism, and one with an unknown function (Table 3).

\section{$\operatorname{miR} 159 / 319$}

It has been previously shown that the miR159 and miR319 families are related in sequence and targets but that divergent expression and slight sequence variation allows for specific biological functions for these distinct miRNAs (Palatnik et al., 2007). After our sequence analysis of $A$. coerulea, it is not possible to determine conclusively if 2183893560 and 2185799162 encode miR159 and/or miR319. However, based solely on their sequences, we predict that 2185799162 encodes aqc-miR319 (Table 1; Fig. 5C). 2183893560 was placed in the miR159 family although its sequence appears to be a hybrid between A. thaliana and O. sativa miR159 and miR319 (Fig. 5C). 2183893560 has a $C T$ at the $7^{\text {th }}$ and $8^{\text {th }}$ position, which is conserved in the A. thaliana and $O$. sativa miR319 family. In contrast it has a TTT repeat at the 5' end and CTCTA at the 3' end, which is more similar to the A. thaliana and $O$. sativa miR159 family (Fig. 5C). No targets were predicted for aqc-miR159 but a novel locus involved in light sensing is predicted as an aqc-miR319 target.

\subsection{Phylogenetic distribution of microRNAs}

Given that $A$. coerulea is a member of the Ranunculales, a lineage that is roughly 
intermediate between the clades that contain A. thaliana and $O$. sativa, we thought it pertinent to evaluate the newly identified $A$. coerulea miRNA families in a phylogenetic context (Fig 4). All A. coerulea miRNA sequences, along with all miRNA sequences from species used as the query in the BLAST searches, were plotted based on presence or absence and number of miRNAs per family (Fig. 6). This analysis produces a clear pattern. A small group of twenty-one miRNA families appear to be highly conserved across angiosperms $(156,159,160,162,164,166,167,168,169,171,172,319,390$, 393, 394, 395, 396, 397, 398, 399 and 408). Axtell and Bowman have previously reached the same conclusion in a similar analysis (Axtell and Bowman, 2008). Of these highly conserved angiosperm miRNA families, we were able to identify 15 of the 21 in A. coerulea (Fig. 6). In addition, conserved miRNA target sites for five of the six uncharacterized miRNA families were identified, suggesting that they too may be present. The high conservation of these 21 miRNA families and their targets is expected given that these miRNAs have been shown to be involved in critical developmental processes, leading us to expect significant pleiotropy and subsequent selection for the conservation of these developmental modules.

In addition to identifying these highly conserved miRNA families in A. coerulea, several miRNA families were predicted that had a more varied phylogenetic distribution (Fig. 6). Axtell and Bowman previously identified 39 miRNA families that are present in two or more distant plant lineages based on miRBASE (version 10.1) (Axtell and Bowman, 2008). Our analysis expands their dataset to 40 conserved miRNA families (Fig. 6) by the addition of miR530, which is conserved in distantly related species: $P$. trichocarpa, A. coerulea, and O. sativa (Lu et al., 2008). To our knowledge, there are currently no predicted targets of miR530 in O. sativa or P. trichocarpa (Liu et al., 2005; 
Lu et al., 2005; Lu et al., 2008), but we have predicted that aqc-miR530 targets a serine carboxypeptidase-like gene. Homologs of this locus were identified in $O$. sativa and $P$. trichocarpa but no miR530-binding site appears to be present in either gene. Moreover, we also included miRNA families that are not as distantly conserved but show representatives across more closely related plant lineages, including two monocot specific miRNA families (miR444 and miR528) (groups previously identified by (Willmann and Poethig, 2007; Sunkar et al., 2008)), a Brassicaceae specific miRNA family (miR824), and three Fabaceae specific miRNA families (mir1507, miR1509, and $\operatorname{miR} 1510)$.

\section{$\operatorname{miR477}$}

miR477 has an interesting phylogenetic distribution as it is present in bryophyes, then absent in the lycopods, gymnosperms, and all surveyed monocots (Axtell et al., 2007), but reported in this paper to be present in Aquilegia (Fig. 6). To our knowledge, in addition to its presence in the bryophyte, $P$. patens, miR477 has only been identified in the core eudicots $P$. trichocarpa and $V$. vinifera (Axtell et al., 2007; Lu et al., 2007). The apparent reappearance of miR477 in Aquilegia, at the base of the eudicots, prompts several interesting questions: (1) what are the targets of miR477 in $P$. patens, (2) are these targets conserved in Aquilegia, V. vinifera, or P. trichocarpa, and (3) what are the characteristics of the target homologs in the lycopods, gymnosperms, monocots, and core eudicots, i.e., is there a remnant of a miR477 binding site? Targets have been predicted for miR477, but to our knowledge these have only been confirmed in P. patens. The only confirmed P.patens miR477 target is a basic helix-loop helix transcription factor (Axtell et al., 2007), while several other $P$. patens miR477 target predictions include an abscisic 
acid-insensitive-like protein and a kelch motif family protein (Axtell et al., 2007). The poplar miR477 has been predicted to target a GRAS domain containing protein, a NAC protein, a zinc finger protein, and a polygalacturonase protein (Lu et al., 2005). Our predicted targets include a plastidic glucose-6-phosphate dehydrogenase, elongation factor 1B-gamma, and an rRNA processing protein (Table 3). We identified homologs of the predicted $A$. coerulea miR477 targets in $P$. trichocarpa, $V$. vinifera, and $P$. patens but no miR477 binding site appears to be present in these genes. In answer to question three, the phylogenetic distance among these taxa and complexity of the target gene families, makes one-to-one comparisons of potential targets challenging. It is notable, however, that while members of the NAC and GRAS gene families are known to be regulated by microRNAs in A. thaliana, they are targeted by other families (miR164 and miR170/171, respectively; Rhoades et al. 2002). Seeing that these regulatory interactions appear to be quite highly conserved (Jones-Rhoades et al., 2006), this would seem to suggest that miR477 acquired targeting of GRAS and NAC family members specifically in $P$. trichocarpa. Similarly, homologs of the predicted Aquilegia targets from rice and $A$. thaliana did not show conservation of the miR477 binding site (data not shown). Thus, further work is needed to confirm miR477 targets and analyze the evolutionary significance of their distribution. Due to the distance between the last common ancestor of $P$. patens and A. coerulea as well as the apparent heterogeneity of miR477 targets, we can imagine two evolutionary scenarios. Under the first, miR477 must be significantly less constrained than other ancient miRNAs in terms of target conservation and even presence in the genome. The other alternative is that this family has actually arisen via convergent evolution, although a mechanism for such convergence remains unclear and the sequence similarity among the predicted miR477 representatives is relatively high 
(Fig. 5D).

$\operatorname{miR529}$

The phylogenetic distribution of miR529 is also quite interesting. miR529 is present in the lycopods, bryophytes, monocots, and the Ranunculids Escscholzia californica and A. coerulea, but is distinctly absent from all core eudicots (Griffiths-Jones et al., 2006; Griffiths-Jones et al., 2008; Barakat et al., 2007) (Fig. 6; see note above regarding lack miR529 in A. thaliana and P. trichocarpa). The fact that miR529 appears to be closely related to miR156 raises the possibility that it may have been lost without ill effect, possibility due to some redundancy with miR156. Before this hypothesis is tested, detailed work comparing miR156 and miR529 and their target specificity, similar to Palatnik et al. (2007) where they determined that subtle sequence and expression differences in the related miR159 and miR319 families led to distinct functions, needs to be performed.

\section{$\operatorname{miR482}$}

Another miRNA family with a disjunct phylogenetic distribution is miR482 (Fig. 6). mir482 has been identified in the eudicot species $G$. max, P. trichocarpa, $V$. viniferia (Barakat et al., 2007; Jaillon et al., 2007; Lu et al., 2008; Zhang et al., 2008a), and now A. coerulea. In addition to its presence in the eudicots, miR482 has only been identified in the gymnosperm $P$. taeda (Lu et al., 2007). In A. coerulea we identified three members of the family but no target predictions were made. Prediction or confirmation of a target is not critical for the annotation of a miRNA and in this case, the inability to identify a target may be due to incomplete coverage in the EST database or, alternatively, it may be due to the fact that miR482 is evolutionary transient (Axtell and Bowman, 2008). Similar 
to the case with miR477, the predicted targets of miR482 are quite diverse. Lu et al. (2005) identified a disease resistance locus in $P$. trichocarpa that is targeted by miR482. We identified a possible Aquilegia homolog of this locus but could not identify a miR482 binding site. Approximately $80 \mathrm{miR} 482$ targets have been predicted in P. taeda, although they were not analyzed in depth due to their large number (Lu et al., 2007). To our knowledge no targets have been predicted for miR482 in G. $\max$.

\section{Conclusions}

This is the first study to systematically identify and annotate miRNAs in the emerging eudicot model Aquilegia. We have identified 45 miRNAs belonging to 20 miRNA families and have determined that, in general, both the miRNA families and predicted targets are highly conserved in Aquilegia when compared to other model plant systems. Also, we have mapped, in a phylogenetic context, what is currently known about the miRNA families in 16 other plant species. Due to Aquilegia's critical phylogenetic position at the approximate midpoint between well developed models $A$. thaliana and $O$. sativa, this data set lays the ground work necessary for further evolutionary and developmental studies on the evolution of miRNAs and their role in the angiosperm diversification.

\section{Acknowledgements}

We thank members of the Kramer lab, Dr. Scott Hodges and \#\# anonymous reviewers for comments on the manuscript. This work was supported by grants NSF-BE \#0412727 and NSF-IBN \#0720240 to EM Kramer. 


\section{References}

Aukerman, M.J., Sakai, H., 2003. Regulation of flowering time and floral organ identity by a MicroRNA and its APETALA2-like target genes. Plant Cell 15, 2730-2741.

Axtell, M.J., Bartel, D.P., 2005. Antiquity of microRNAs and their targets in land plants. Plant Cell 17, 1658-1673.

Axtell, M.J., Bowman, J.L., 2008. Evolution of plant microRNAs and their targets. Trends Plant Sci. 13, 343-349.

Axtell, M.J., Snyder, J.A., Bartel, D.P., 2007. Common functions for diverse small RNAs of land plants. Plant Cell 19, 1750-1769.

Barakat, A., Wall, P.K., Diloreto, S., Depamphilis, C.W., Carlson, J.E., 2007. Conservation and divergence of microRNAs in Populus. BMC Genomics 8, 481. Barakat, A., Wall, K., Leebens-Mack, J., Wang, Y.J., Carlson, J.E.Depamphilis, C.W., 2007. Large-scale identification of microRNAs from a basal eudicot (Eschscholzia californica) and conservation in flowering plants. Plant J. 51, 9911003.

Boualem, A., Laporte, P., Jovanovic, M., Laffont, C., Plet, J., Combier, J.P., Niebel, A., Crespi, M., Frugier, F., 2008. MicroRNA166 controls root and nodule development in Medicago truncatula. Plant J. 54, 876-887.

Cartolano, M., Castillo, R., Efremova, N., Kuckenberg, M., Zethof, J., Gerats, T., Schwarz-Sommer, Z., Vandenbussche, M., 2007. A conserved microRNA module exerts homeotic control over Petunia hybrida and Antirrhinum majus floral organ identity. Nat. Genet. 39, 901-905. 
Chaw, S.M., Chang, C.C., Chen, H.L., Li, W.H., 2004. Dating the monocot-dicot divergence and the origin of core eudicots using whole chloroplast genomes. J. Mol. Evol. 58, 424-441.

Chen, X., 2004. A microRNA as a translational repressor of APETALA2 in Arabidopsis flower development. Science 303, 2022-2025.

Ding, D., Zhang, L., Wang, H., Liu, Z., Zhang, Z., Zheng, Y., 2009. Differential expression of miRNAs in response to salt stress in maize roots. Ann. Bot. (Lond.) $103,29-38$.

Fahlgren, N., Howell, M.D., Kasschau, K.D., Chapman, E.J., Sullivan, C.M., Cumbie, J.S., Givan, S.A., Law, T.F., Grant, S.R., Dangl, J.L., et al., 2007. Highthroughput sequencing of Arabidopsis microRNAs: evidence for frequent birth and death of MIRNA genes. PLoS ONE 2, e219.

Gandikota, M., Birkenbihl, R.P., Hohmann, S., Cardon, G.H., Saedler, H., Huijser, P., 2007. The miRNA156/157 recognition element in the 3' UTR of the Arabidopsis SBP box gene SPL3 prevents early flowering by translational inhibition in seedlings. Plant J. 49, 683-693.

Griffiths-Jones, S., 2004. The microRNA Registry. Nucleic Acids Res. 32, D109-111.

Griffiths-Jones, S., Grocock, R.J., van Dongen, S., Bateman, A.Enright, A.J., 2006. miRBase: microRNA sequences, targets and gene nomenclature. Nucleic Acids Res. 34, D140-144.

Griffiths-Jones, S., Saini, H.K., van Dongen, S., Enright, A.J., 2008. miRBase: tools for microRNA genomics. Nucleic Acids Res. 36, D154-158. 
Guo, A.Y., Zhu, Q.H., Gu, X., Ge, S., Yang, J., Luo, J., 2008. Genome-wide identification and evolutionary analysis of the plant specific SBP-box transcription factor family. Gene 418, 1-8.

Hodges, S.A., Arnold, M.L., 1994a. Columbines - a Geographically Widespread Species Flock. Proc. Natl. Acad. Sci. 91, 5129-5132.

Hodges, S.A., Arnold, M.L., 1994b. Floral and ecological isolation between Aquilegia formosa and Aquilegia pubescens. Proc. Natl. Acad. Sci. U S A 91, 2493-2496.

Hodges, S.A., Fulton M., Yang JY, Whittal JB., 2003. Verne Grant and evolutionary studies of Aquilegia. New Phytologist 113-120.

Hodges, S.A., Kramer, E.M., 2007. Columbines. Curr. Biol. 17, R992-994.

Jaillon, O., Aury, J.M., Noel, B., Policriti, A., Clepet, C., Casagrande, A., Choisne, N., Aubourg, S., Vitulo, N., Jubin, C., et al., 2007. The grapevine genome sequence suggests ancestral hexaploidization in major angiosperm phyla. Nature 449, 463467.

Jones-Rhoades, M.W., Bartel, D.P., 2004. Computational identification of plant microRNAs and their targets, including a stress-induced miRNA. Mol. Cell 14, 787-799.

Jones-Rhoades, M.W., Bartel, D.P., Bartel, B., 2006. MicroRNAS and their regulatory roles in plants. Annu. Rev. Plant. Biol. 57, 19-53.

Lauter, N., Kampani, A., Carlson, S., Goebel, M., Moose, S.P., 2005. microRNA172 down-regulates glossy15 to promote vegetative phase change in maize. Proc. Natl. Acad. Sci. U S A 102, 9412-9417. 
Liu, B., Li, P., Li, X., Liu, C., Cao, S., Chu, C., Cao, X., 2005. Loss of function of OsDCL1 affects microRNA accumulation and causes developmental defects in rice. Plant Physiol. 139, 296-305.

Lu, S., Sun, Y.H., Amerson, H., Chiang, V.L., 2007. MicroRNAs in loblolly pine (Pinus taeda L.) and their association with fusiform rust gall development. Plant J. 51, 1077-1098.

Lu, S., Sun, Y.H., Chiang, V.L., 2008. Stress-responsive microRNAs in Populus. Plant J. $55,131-151$.

Lu, S., Sun, Y.H., Shi, R., Clark, C., Li, L.Chiang, V.L., 2005. Novel and mechanical stress-responsive MicroRNAs in Populus trichocarpa that are absent from Arabidopsis. Plant Cell 17, 2186-2203.

Mathews, D.H., Sabina, J., Zuker, M., Turner, D.H., 1999. Expanded sequence dependence of thermodynamic parameters improves prediction of RNA secondary structure. J. Mol. Biol. 288, 911-940.

Meyers, B.C., Axtell, M.J., Bartel, B., Bartel, D.P., Baulcombe, D., Bowman, J.L., Cao, X., Carrington, J.C., Chen, X., Green, P.J., et al., 2008. Criteria for Annotation of Plant MicroRNAs. Plant Cell

Mi, S., Cai, T., Hu, Y., Chen, Y., Hodges, E., Ni, F., Wu, L., Li, S., Zhou, H., Long, C., et al., 2008. Sorting of small RNAs into Arabidopsis argonaute complexes is directed by the 5 ' terminal nucleotide. Cell 133, 116-127.

Montgomery, T.A., Howell, M.D., Cuperus, J.T., Li, D., Hansen, J.E., Alexander, A.L., Chapman, E.J., Fahlgren, N., Allen, E., Carrington, J.C., 2008. Specificity of ARGONAUTE7-miR390 interaction and dual functionality in TAS3 trans-acting siRNA formation. Cell 133, 128-141. 
Moore, M.J., Bell, C.D., Soltis, P.S., Soltis, D.E., 2007. Using plastid genome-scale data to resolve enigmatic relationships among basal angiosperms. Proc. Natl. Acad. Sci. 104, 19363-19368.

Palatnik, J.F., Allen, E., Wu, X., Schommer, C., Schwab, R., Carrington, J.C.Weigel, D., 2003. Control of leaf morphogenesis by microRNAs. Nature 425, 257-263.

Palatnik, J.F., Wollmann, H., Schommer, C., Schwab, R., Boisbouvier, J., Rodriguez, R., Warthmann, N., Allen, E., Dezulian, T., Huson, D., et al., 2007. Sequence and expression differences underlie functional specialization of arabidopsis microRNAs miR159 and miR319. Dev. Cell 13, 115-125.

Paterson, A.H., Bowers, J.E., Bruggmann, R., Dubchak, I., Grimwood, J., Gundlach, H., Haberer, G., Hellsten, U., Mitros, T., Poliakov, A., et al., 2009. The Sorghum bicolor genome and the diversification of grasses. Nature 457, 551-556.

Rhoades, M.W., Reinhart, B.J., Lim, L.P., Burge, C.B., Bartel, B., Bartel, D.P., 2002. Prediction of plant microRNA targets. Cell 110, 513-520.

Sanderson, M.J., Thorne, J.L., Wikstrom, N., Bremer, K., 2004. Molecular evidence on plant divergence times. American Journal of Botany 91, 1656-1665.

Schwab, R., Ossowski, S., Riester, M., Warthmann, N., Weigel, D., 2006. Highly specific gene silencing by artificial microRNAs in Arabidopsis. Plant Cell 18, 1121-1133.

Schwab, R., Palatnik, J.F., Riester, M., Schommer, C., Schmid, M., Weigel, D., 2005. Specific effects of microRNAs on the plant transcriptome. Dev. Cell 8, 517-527. Shukla, L.I., Chinnusamy, V., Sunkar, R., 2008. The role of microRNAs and other endogenous small RNAs in plant stress responses. Biochim. Biophys. Acta 1779, 743-748. 
Sunkar, R., Zhou, X., Zheng, Y., Zhang, W., Zhu, J.K., 2008. Identification of novel and candidate miRNAs in rice by high throughput sequencing. BMC Plant Biol. 8, 25.

Takeda, A., Iwasaki, S., Watanabe, T., Utsumi, M., Watanabe, Y., 2008. The mechanism selecting the guide strand from small RNA duplexes is different among argonaute proteins. Plant Cell Physiol. 49, 493-500.

Usami, T., Horiguchi, G., Yano, S., Tsukaya, H., 2009. The more and smaller cells mutants of Arabidopsis thaliana identify novel roles for SQUAMOSA PROMOTER BINDING PROTEIN-LIKE genes in the control of heteroblasty. Development 136, 955-964.

Wang, J.W., Schwab, R., Czech, B., Mica, E., Weigel, D., 2008. Dual effects of miR156targeted SPL genes and CYP78A5/KLUH on plastochron length and organ size in Arabidopsis thaliana. Plant Cell 20, 1231-1243.

Willmann, M.R., Poethig, R.S., 2007. Conservation and evolution of miRNA regulatory programs in plant development. Curr. Opin. Plant Biol. 10, 503-511.

Wu, M.F., Tian, Q., Reed, J.W., 2006. Arabidopsis microRNA167 controls patterns of ARF6 and ARF8 expression, and regulates both female and male reproduction. Development 133, 4211-4218.

Zhang, B., Pan, X., Cannon, C.H., Cobb, G.P., Anderson, T.A., 2006a. Conservation and divergence of plant microRNA genes. Plant J. 46, 243-259.

Zhang, B., Pan, X., Stellwag, E.J., 2008a. Identification of soybean microRNAs and their targets. Planta 229, 161-182.

Zhang, B., Wang, Q., Wang, K., Pan, X., Liu, F., Guo, T., Cobb, G.P., Anderson, T.A., 2007. Identification of cotton microRNAs and their targets. Gene 397, 26-37. 
Zhang, B.H., Pan, X.P., Cox, S.B., Cobb, G.P., Anderson, T.A., 2006b. Evidence that miRNAs are different from other RNAs. Cell Mol. Life Sci. 63, 246-254.

Zhang, J.F., Yuan, L.J., Shao, Y., Du, W., Yan, D.W., Lu, Y.T., 2008b. The disturbance of small RNA pathways enhanced abscisic acid response and multiple stress responses in Arabidopsis. Plant Cell Environ. 31, 562-574.

Zuker, M., 2003. Mfold web server for nucleic acid folding and hybridization prediction. Nucleic Acids Res. 31, 3406-3415. 


\section{Figure Legends}

Fig. 1. Simplified phylogeny and divergence times of the land plants based on (Sanderson, 2003; Chaw et al., 2004; Sanderson et al., 2004; Moore et al., 2007; Qiu et al., 2007; Rensing et al., 2008). Major model systems associated with the various land plant lineages are listed.

Fig. 2 Flow diagram illustrating protocol used to identify conserved Aquilegia miRNAs.

Fig. 3. Position-specific nucleotide distribution among A. coerulea mature miRNA. Percentage distribution of nucleotides at position 1-21 of mature A. coerulea miRNA. The average percentage nucleotide composition is depicted as average.

Fig. 4. Three Aquilegia coerulea miRNA predicted precursor hairpin structures chosen as a representative of all A. coreulea miRNAs identified. All predicted precursor hairpin structures are available in the supplementary information.

Fig. 5. miRNA families discussed in greater detail in the text (A) Hairpin structure of gi20218562092, which appears to encode miR156 and/or miR529. The gray line delineates the miR156 mature sequence while the black line delineates the miR529 mature sequence. (B) Alignment of miR156/miR529 families of A. coerulea (2185892723, 2185805752, 2185518132, and 2185620929), O. sativa, A. thaliana, and $P$. patens. (C) Alignment of miR159/miR319 families of A. coerulea (2183893560 and 2185799162), O. sativa, and A. thaliana. A. (D) Alignment of miR477 families of $A$. coerulea $(2185477245,2185552337,2185755300,2185768332,2185874405$, 2185853634, and 2185809227), P. trichocarpa, V. vinifera, and P. patens For B-D, osa: 
Oryza sativa, ath: Arabidopsis thaliana, ptc: Populus trichocarpa, ppt: Physcomitrella patens, vvi: Vitis vinifera.

Fig. 6. Phylogenetic distribution of plant miRNAs. All plant miRNAs conserved in two or more plant species [as determined by miRBASE (Release 13.0) and the recently released S. bicolor genome (Paterson et al., 2009)] are plotted. Phylogenetic affinities of each taxon are indicated by the colored bars on the left: $\operatorname{Red}=$ core eudicot; Green = eudicot; Dark Blue $=$ monocot $;$ Yellow $=$ gymnosperm $;$ Pink $=$ lycophyte $;$ and Light Blue $=$ bryophyte. Shading of the boxes represent our findings: dark gray $=$ miRNA present, light gray $=m i R N A$ target sequence identified, dotted $=$ No target identified. Numbers in boxes represent the number of miRNAs present in a particular miRNA family. $\dagger$ indicates that a miRNA sequence was reported by (Sunkar and Jagadeeswaran, 2008) from the Aquilegia EST database. We searched the EST database for miR394 but failed to identify it, presumably because of stricter annotation criteria recently described in (Meyers et al., 2008). 
Table 1

Conserved Aquilegia coerulea microRNAs

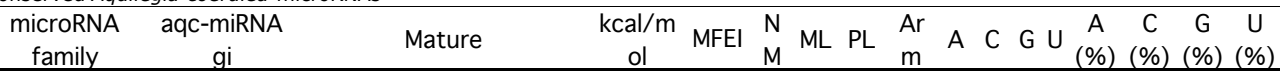

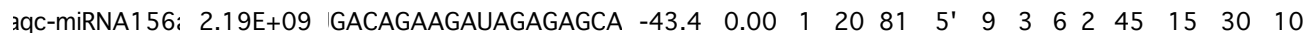

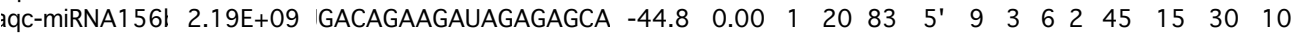

aqc-miRNA159 2.18E+09 UUGGACUGAAGGGAGCUCL

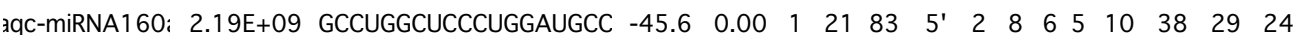

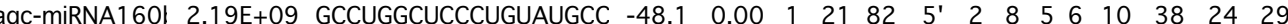

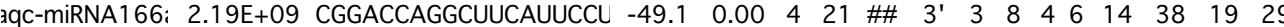

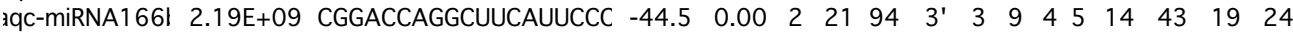

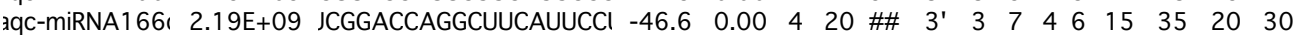

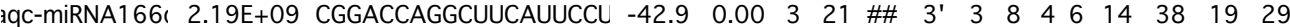

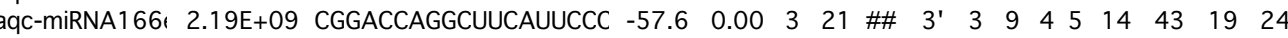

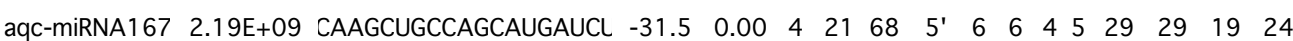
aqc-miRNA168 2.19E+09 GGCUUAGUGCAGCUCGGGG $-40.5 \quad 0.00 \quad 4 \quad 21 \quad \# \# \quad 5 ' \quad 3 \quad 4 \quad 9 \quad 5 \quad 14 \quad 1943 \quad 24$

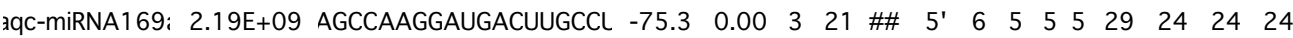

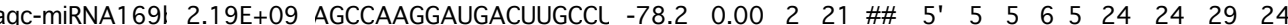

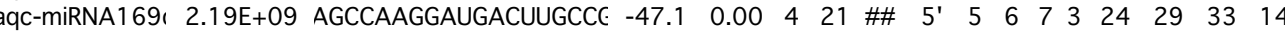

aqc-miRNA171: 2.19E+09 GAUUGAGCCGUGCCAAUAL

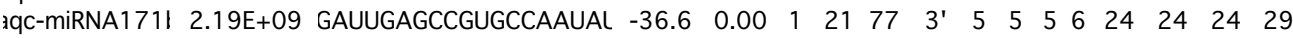

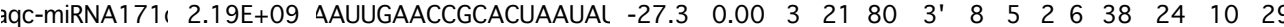

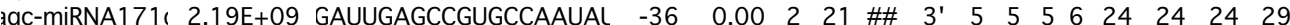

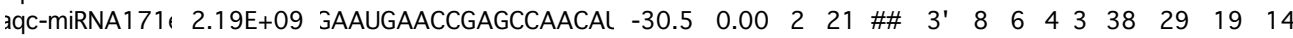

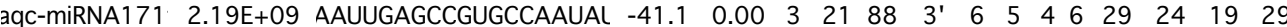

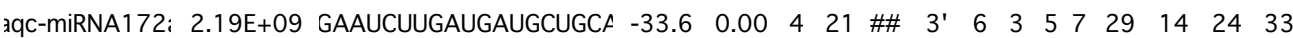

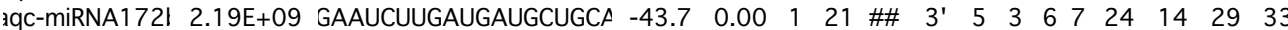

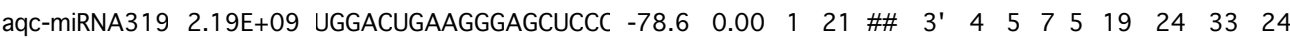

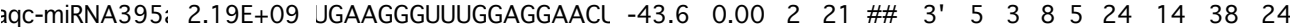

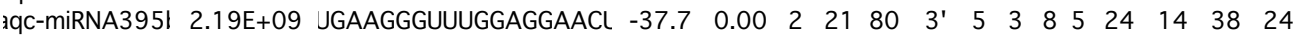

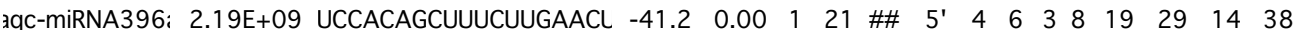

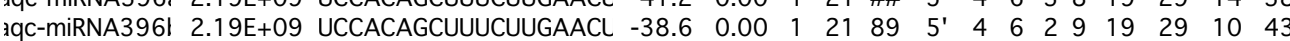

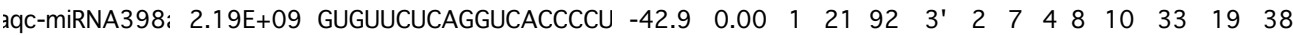
aqc-miRNA3981 2.19E+09 GUGUUCUCAGGUCGCCCCU $\begin{array}{llllllllllllll}-38.5 & 0.00 & 1 & 21 & 93 & 3^{\prime} & 1 & 7 & 6 & 7 & 5 & 33 & 29 & 33\end{array}$

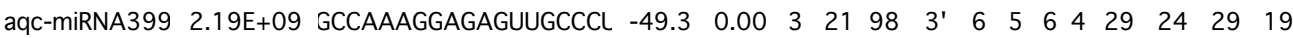

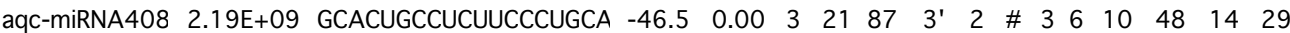

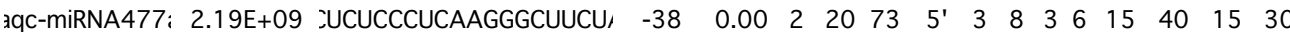
aqc-miRNA4771 $2.19 E+09$ :UCUCCCUCAAGGGCUUCU/

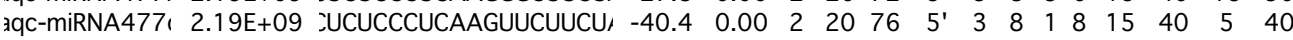

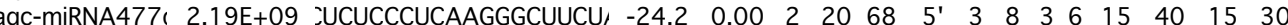

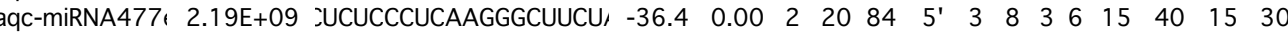

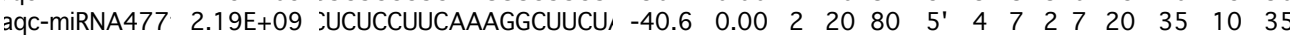
xqc-miRNA477، 2.19E+09 :UCUCCCUCAAGUUCUUCUt

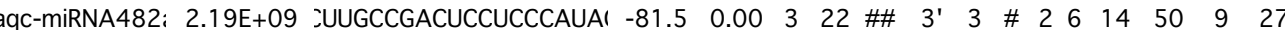

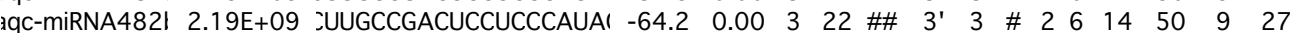

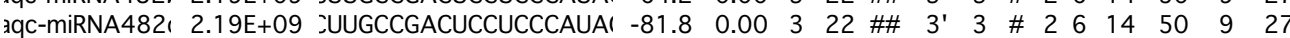
aqc-miRNA529 2.19E+09 JAAGAGAGAGAGCACAAC( $-60 \quad 0.00 \quad 1 \quad 21$ \#\# 5 5' \# $5 \begin{array}{llllllll}6 & 0 & 48 & 24 & 29 & 0\end{array}$

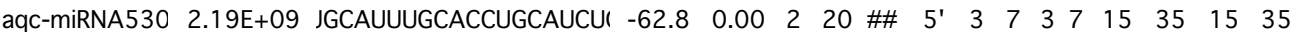

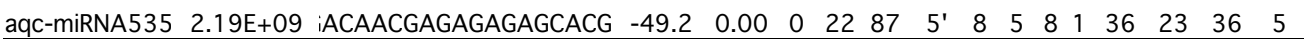

gi: gene index number corresponding to $A$. coerulea whole genome sequence database MFEI: minimal folding free energy index

NM: number of mismatches in miRNA/miRNA* duplex

ML: Mature Length

PL: Precursor Length

ARM: Location of mature miRNA ( $3^{\prime}$ or $\left.5^{\prime}\right)$ 
Table 2

miRNA precursor

characteristics Minimum Maximum Average Std Dev

\begin{tabular}{lllll}
\hline Sequence Length & 68 & 180 & 106 & 32.2
\end{tabular}

A (\%)

$\begin{array}{llll}16.4 & 36.3 & 25.6 & 4.4\end{array}$

$\mathrm{U}(\%)$

$\mathrm{G}(5)$

$\begin{array}{llll}19.7 & 38.1 & 31.4 & 3.8\end{array}$

$C(\%)$

$\begin{array}{llll}13.8 & 31.5 & 22.9 & 3.7\end{array}$

MFE $(-\mathrm{kcal} / \mathrm{mol})$

13.5

$29.9 \quad 20.1 \quad 3.0$

AMFE $(-\mathrm{kcal} / \mathrm{mol})$

18.8

$\begin{array}{lll}81.8 & 46 & 14.7\end{array}$

MFEI

26.5

$58.7 \quad 44.1$

7.5

0.60

1.48

1.03

0.18 


\begin{tabular}{|c|c|c|c|c|}
\hline nicroRNA family & Targeted Protein & Target Function & TC\# & Top Scoring A.t. locu \\
\hline \multirow[t]{7}{*}{156} & Squamosa promoter binding protein-like 2 & Transcription factor & TC24142 & AT5643270 \\
\hline & $\begin{array}{l}\text { Squamosa promoter binding protein-like } 6 \\
\text { (SBP) }\end{array}$ & Transcription factor & $\begin{array}{l}\text { TC26823, TC21170, } \\
\text { TC21808, } \\
\text { DR954092 }\end{array}$ & AT1G69170 \\
\hline & $\begin{array}{l}\text { Squamosa promoter binding protein-like } 13 \\
\text { (SBP) }\end{array}$ & Transcription factor & $\begin{array}{l}\text { TC32167, } \\
\text { DR949672 }\end{array}$ & AT5650570 \\
\hline & $\begin{array}{l}\text { Squamosa promoter binding protein-like } 14 \\
\text { (SBP) }\end{array}$ & Transcription factor & TC21707 & AT1G20980 \\
\hline & $\begin{array}{l}\text { Squamosa promoter binding protein-like } 12 \\
\text { (SBP) }\end{array}$ & Transcription factor & TC31356 & AT3G60030 \\
\hline & Growth Regulating Factor 2 (GRF2) & Transcription factor & TC25281 & AT4G37740 \\
\hline & Unknown & Unknown & TC31827 & AT1G40133 \\
\hline 159 & None & & & \\
\hline 160 & $\begin{array}{l}\text { Transposable element } \\
\text { Psuedo-response regulator } 2 \text { (APRR2) }\end{array}$ & Transcription factor & $\begin{array}{l}\text { DT734946 } \\
\text { TC22019 }\end{array}$ & $\begin{array}{l}\text { AT5G28526 } \\
\text { AT4G18020 }\end{array}$ \\
\hline 162 & None & & & \\
\hline \multirow[t]{4}{*}{164} & Cup-Shaped Cotyledon 2 (CUC2) & Transcription factor & TC27953 & AT5G53950 \\
\hline & NAC Domain containing protein & Transcription factor & TC27854 & AT5639610 \\
\hline & $\begin{array}{l}\text { Leucine-rich repeat transmembrane protein } \\
\text { kinase }\end{array}$ & Metabolism & DT731408 & AT3G28040 \\
\hline & $\begin{array}{l}\text { UDP-qucose pyrophosphorylase } \\
\text { 4-coumarate-CoA ligase family protein }\end{array}$ & $\begin{array}{l}\text { Stress } \\
\text { Stress }\end{array}$ & $\begin{array}{l}\text { TC21677 } \\
\text { DT741735, } \\
\text { TC24498 }\end{array}$ & $\begin{array}{l}\text { AT3G03250 } \\
\text { AT1G20510 }\end{array}$ \\
\hline 166 & Class III HD-Zip protein & Transcription factor & TC30761 & AT1G52150 \\
\hline 167 & Auxin response factor 6 & Transcription factor & TC29952, TC24356 & AT1630330 \\
\hline 168 & $\begin{array}{l}\text { Unknown } \\
\text { Anion Transporter } 2\end{array}$ & $\begin{array}{l}\text { Metabolism } \\
\text { Transport }\end{array}$ & $\begin{array}{l}\text { DT747736 } \\
\text { TC29087 }\end{array}$ & $\begin{array}{l}\text { AT3G48870 } \\
\text { AT4600370 }\end{array}$ \\
\hline \multirow[t]{2}{*}{169} & CCAAT-binding factor (HAP2) & Transcription factor & DR924236 & AT1G72830 \\
\hline & Lipase class 3 family protein & Metabolsim & TC31138 & AT5618640 \\
\hline \multirow[t]{2}{*}{$170 / 171$} & Scarecrow-like protein & Transcription factor & $\begin{array}{l}\text { TC21487, TC28359, } \\
\text { TC25185 }\end{array}$ & AT2G45160 \\
\hline & Arabidopsis Pumilio 2 (APUM2)-like & RNA bindina & TC29397 & AT2G29190 \\
\hline \multirow{7}{*}{172} & APETALA2 & Transcription factor & TC24245 & AT4636920 \\
\hline & Target of EAT 1 (TOE1) & Transcription factor & TC28449, TC32032 & AT2628550 \\
\hline & $\begin{array}{l}\text { Unknown } \\
\text { Unknown }\end{array}$ & $\begin{array}{l}\text { Unknown } \\
\text { protein binding, zinc }\end{array}$ & $\begin{array}{l}\text { DR954144 } \\
\text { TC26960 }\end{array}$ & $\begin{array}{l}\text { AT5G65290 } \\
\text { AT3G48070 }\end{array}$ \\
\hline & Spike1 (SPR1) & $\begin{array}{l}\text { Metabolism } \\
\text { Mats }\end{array}$ & DT764297 & AT4G16340 \\
\hline & $\begin{array}{l}\text { Unknown } \\
\text { Unpicina tactor }\end{array}$ & $\begin{array}{l}\text { Metabolism factor } \\
\text { Transcription factor }\end{array}$ & DT749684 & AT3G10070 \\
\hline & $\begin{array}{l}\text { Ubitiquitin-coniuaatina enzyme } \\
\text { F-box protein }\end{array}$ & $\begin{array}{l}\text { Metabolism } \\
\text { Photooprocesses }\end{array}$ & $\begin{array}{l}\text { TC21155 } \\
\text { TC28027 }\end{array}$ & $\begin{array}{l}\text { AT5G05080 } \\
\text { AT4G02440 }\end{array}$ \\
\hline & Selenium bindina protein & Stess & TC29593 & AT2624440 \\
\hline 319 & $\begin{array}{l}\text { Diminuto 1, Enhanced Very-Low-Fluence } \\
\text { Resoonses } 1\end{array}$ & Photoprocesses & TC25461 & AT3G19820 \\
\hline 390 & Cation symporter & Transport & TC30528 & AT5G03560 \\
\hline 393 & $\begin{array}{l}\text { Auxina Sianalizna F-BOX } 2 \\
\text { Transport Inhibitor Response } 1 \text { (TIR1) }\end{array}$ & $\begin{array}{l}\text { Auxin } \\
\text { Auxin }\end{array}$ & $\begin{array}{l}\text { TC29517 } \\
\text { TC20718, TC25434 }\end{array}$ & $\begin{array}{l}\text { AT3G26810 } \\
\text { AT3G62980 }\end{array}$ \\
\hline 394 & F-box protein & Unknown & TC24293 & AT1G27340 \\
\hline 395 & $\begin{array}{l}\text { Kinase } \\
\text { Unknown } \\
\text { ATP sulfurylase }\end{array}$ & $\begin{array}{l}\text { Metabolism } \\
\text { Unknown } \\
\text { Stress }\end{array}$ & $\begin{array}{l}\text { TC32489 } \\
\text { TC20934 } \\
\text { TC21492 }\end{array}$ & $\begin{array}{l}\text { AT4G00955 } \\
\text { AT3G45950 } \\
\text { AT3G22890 }\end{array}$ \\
\hline & Unknown & Unknown & TC30052 & AT2G02370 \\
\hline \multirow[t]{4}{*}{396} & Unknown & Unknown & DR926810 & AT2G16760 \\
\hline & $\begin{array}{l}\text { Calmoduluin-bindinan protein } \\
\text { Mildew Resistance Locus }\end{array}$ & $\begin{array}{l}\text { Stress } \\
\text { Stress }\end{array}$ & $\begin{array}{l}\text { TC29396 } \\
\text { TC32094 }\end{array}$ & $\begin{array}{l}\text { AT3G169440 } \\
\text { AT1G1000 }\end{array}$ \\
\hline & 1-aminocyclopropane-1-carboxylate & Stress & TC20841 & AT1G05010 \\
\hline & UDP-alucosvil transferase family protein & Stress & TC22590 & AT2G18560 \\
\hline 397 & Laccase & Metabolism & TC26926 & AT5G01190 \\
\hline & Laccase & Metabolism & TC26200 & AT2G38080 \\
\hline & $\begin{array}{l}\text { Yip1 family protein } \\
\text { Unknown }\end{array}$ & $\begin{array}{l}\text { Unknown } \\
\text { Unknown }\end{array}$ & $\begin{array}{l}\text { TC24152 } \\
\text { DT728841 }\end{array}$ & $\begin{array}{l}\text { AT2G39805 } \\
\text { AT5G53740 }\end{array}$ \\
\hline 398 & None & & & \\
\hline 399 & $\begin{array}{l}\text { beta-fructofuranosidase } \\
\text { ABA receptor }\end{array}$ & $\begin{array}{l}\text { Metabolism } \\
\text { Metabolism }\end{array}$ & $\begin{array}{l}\text { TC28894 } \\
\text { TC20517 }\end{array}$ & $\begin{array}{l}\text { AT1G56560 } \\
\text { AT2G20770 }\end{array}$ \\
\hline 408 & Xvloglucan Endotransalvcosvlase 6 & Metabolism & TC30505 & AT4G25810 \\
\hline \multirow[t]{3}{*}{477} & Plastidic glucose-6-phosphate & Metabolism & TC30732 & AT5G35790 \\
\hline & $\begin{array}{l}\text { Elongation Factor 1B-qamma } \\
\text { Elonger }\end{array}$ & Metab & $\mathrm{TC}$ & AT1609640 \\
\hline & rRNA processina protein & Metabolism & TC24529 & AT5G61330 \\
\hline 482 & None & & & \\
\hline \multirow[t]{8}{*}{529} & $\begin{array}{l}\text { Squamosa promoter binding protein-like } 6 \\
\text { (SBP) }\end{array}$ & Transcription factor & $\begin{array}{l}\text { DR954092, } \\
\text { TC26823, TC21808, } \\
\text { TC21170 }\end{array}$ & AT1G69170 \\
\hline & $\begin{array}{l}\text { Squamosa promoter binding protein-like } 13 \\
\text { (SBP) }\end{array}$ & Transcription factor & $\begin{array}{l}\text { DR949672, } \\
\text { TC32167 }\end{array}$ & AT5650570 \\
\hline & $\begin{array}{l}\text { Osephin family protein } \\
\text { Squamosa promoter binding protein-like } 14\end{array}$ & $\begin{array}{l}\text { Unknown } \\
\text { Transcription factor }\end{array}$ & $\begin{array}{l}\text { TC20719 } \\
\text { TC271707 }\end{array}$ & $\begin{array}{l}\text { AT3G54130 } \\
\text { AT1620980 }\end{array}$ \\
\hline & (SBP) & & & \\
\hline & $\begin{array}{l}\text { Squamosa promoter binding protein-like } 2 \\
\end{array}$ & Transcription factor & TC24142 & AT5G43270 \\
\hline & Threonine endopeptidase & Unknown & TC32968 & AT4G01150 \\
\hline & FAD-bindina domain-containina protein & Metabolism & DR933604 & AT4G20800 \\
\hline & $\begin{array}{l}\text { Diacylalycerol kinase eqene tamily } \\
\text { Metal Tolerance Protein A2 }\end{array}$ & $\begin{array}{l}\text { Stress } \\
\text { Stress }\end{array}$ & $\begin{array}{l}\text { TC29483 } \\
\text { TC24319 }\end{array}$ & $\begin{array}{l}\text { AT5G63770 } \\
\text { AT3G5810 }\end{array}$ \\
\hline 530 & Serine carboxypeptidase-like 26 & Metabolism & TC23269 & AT2G35780 \\
\hline 535 & None & & & \\
\hline \multirow[t]{3}{*}{783} & $\begin{array}{l}\text { Phosshoenolopruvate carboxylase } \\
\text { Methionine gamma-lyase }\end{array}$ & $\begin{array}{l}\text { Metabolism } \\
\text { Metabolism }\end{array}$ & $\begin{array}{l}\text { DR942629 } \\
\text { TC32408, TC27593 }\end{array}$ & $\begin{array}{l}\text { AT3G14940 } \\
\text { AT1G64660 }\end{array}$ \\
\hline & Glycos & Metab & DT65875 & AT5G \\
\hline & Carrier family protein & Transport & TC33228 & AT4 \\
\hline
\end{tabular}




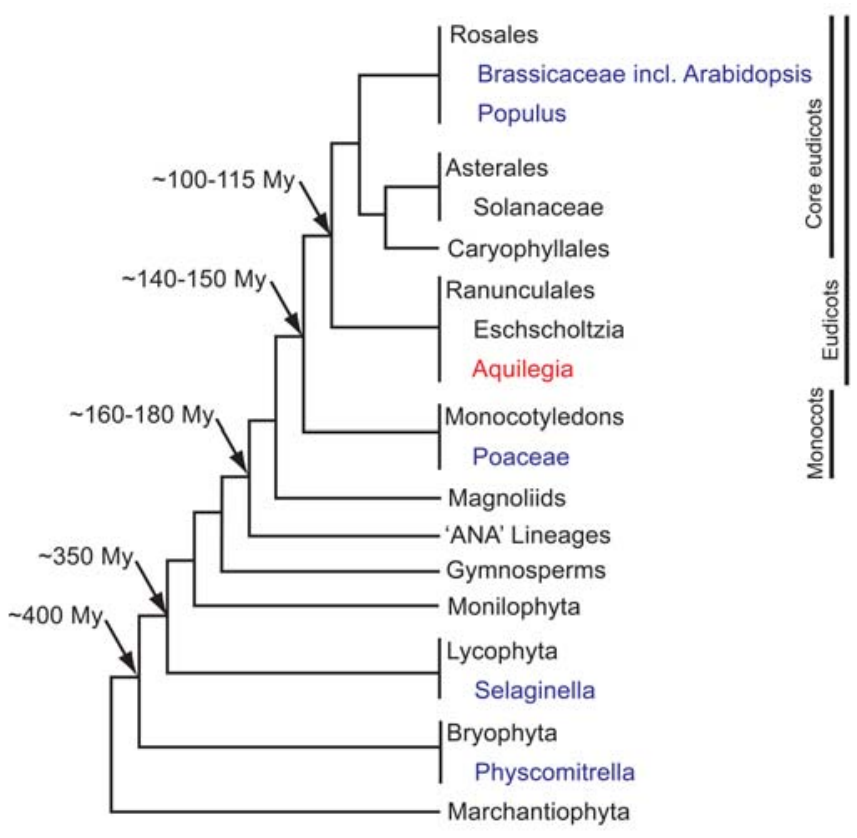




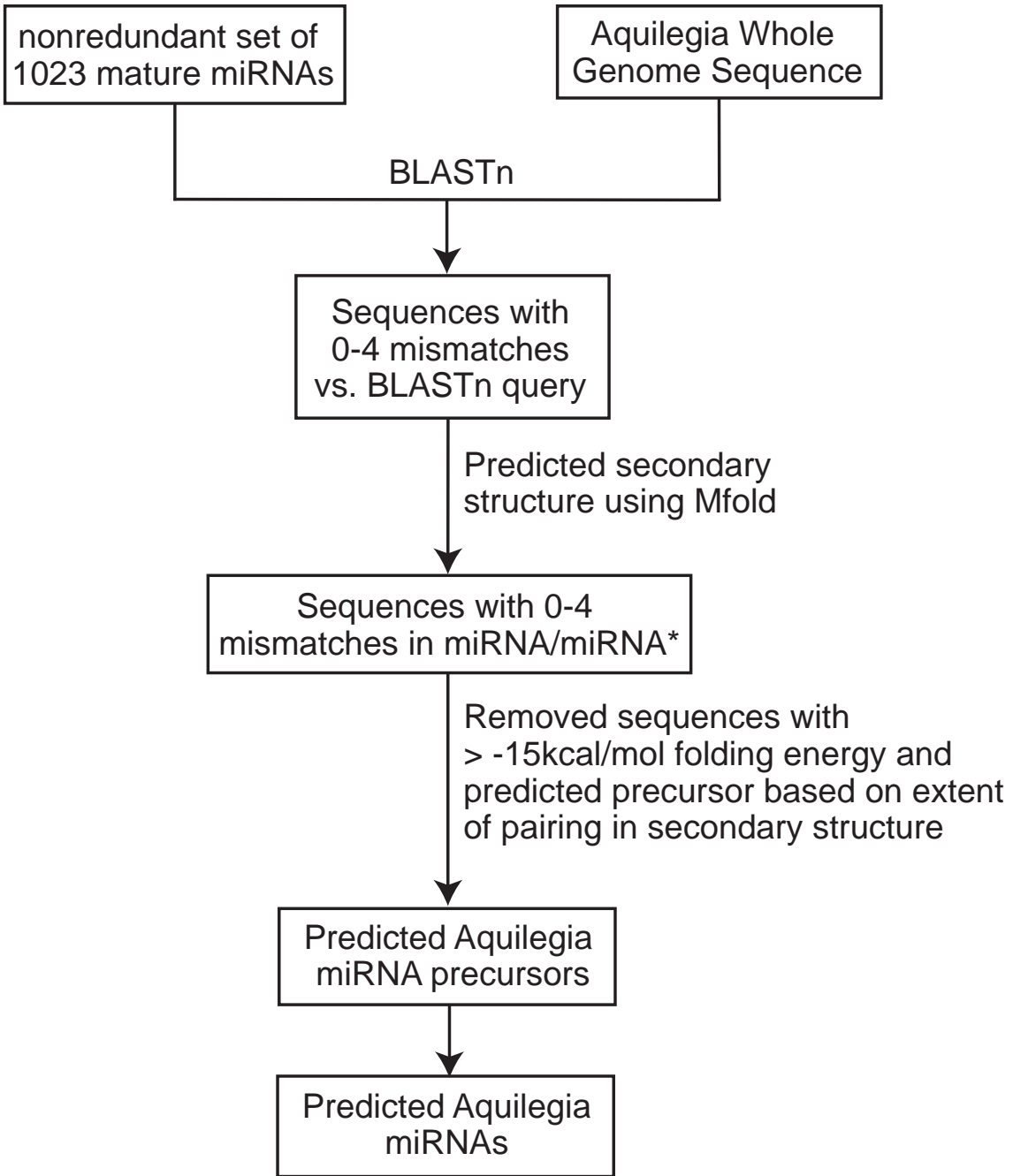




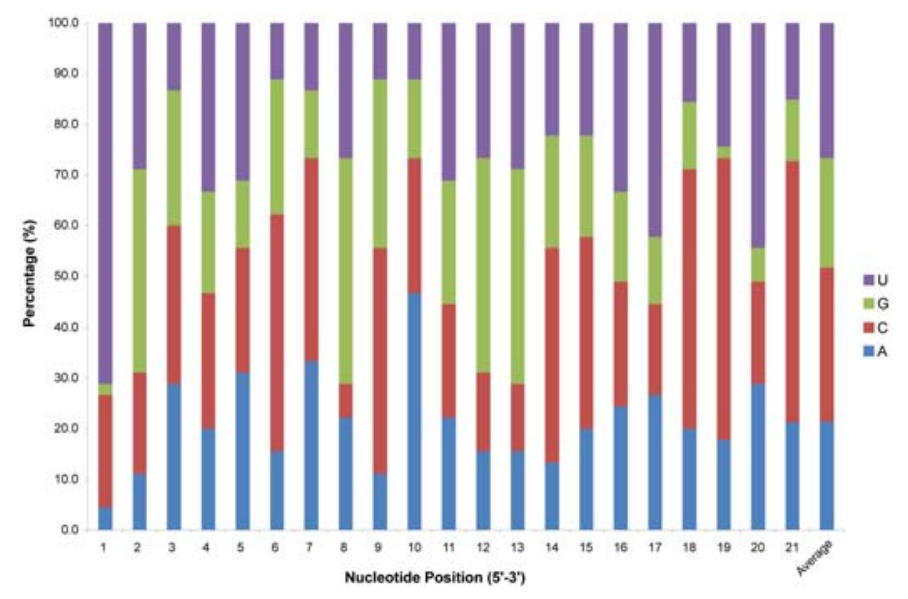



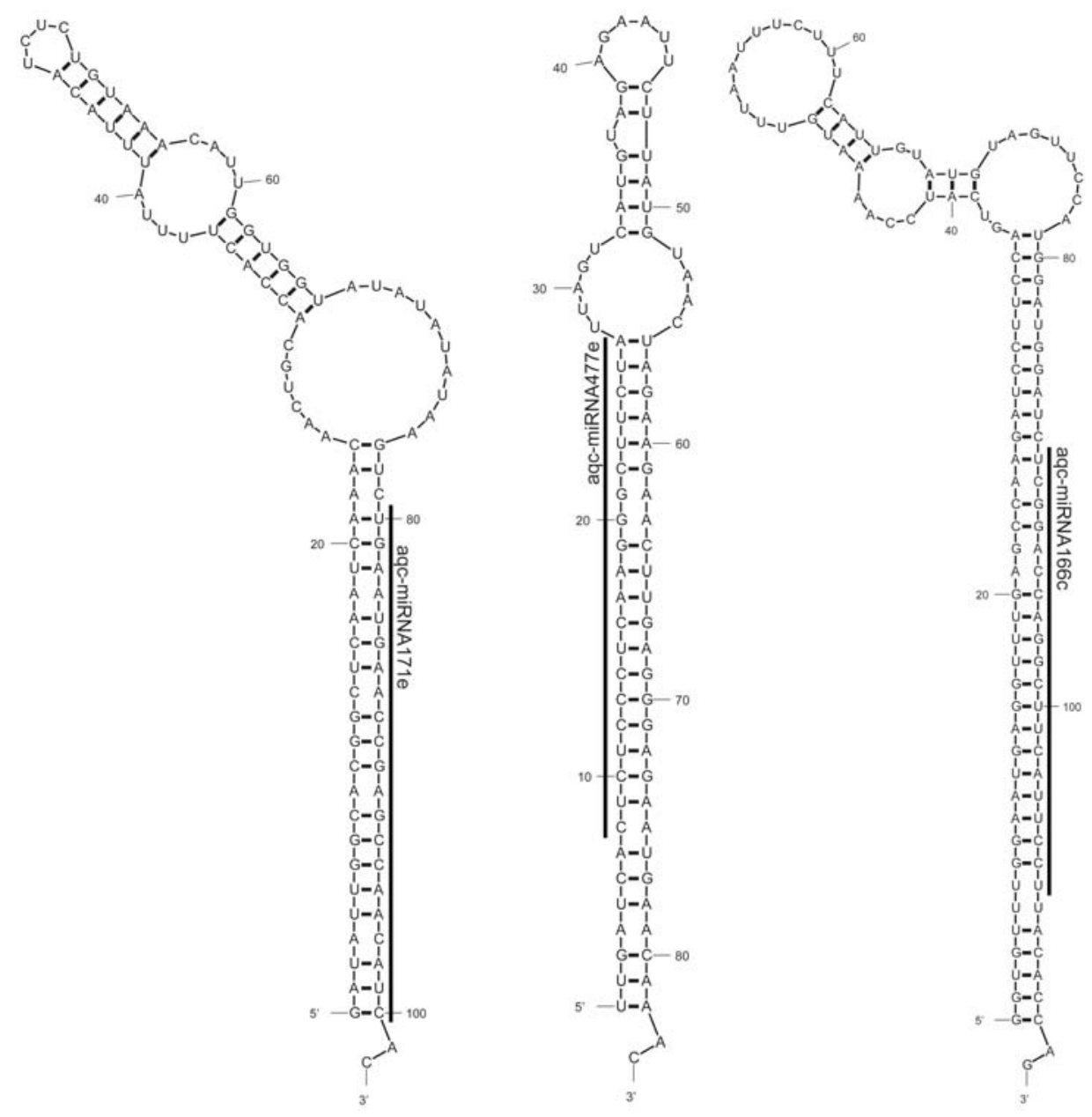
A.<smiles>[R16][R16]([H])([H])[H]</smiles>

B.

ath-miR156a osa-miR156a osa-miR156b ath-miR156c ath-miR156b osa-miR156c 2185892723 2185805752 2185518132 2185620929 osa-miR529b ppt-miR529e ppt-miR529f ppt-miR529g ppt-miR529a ppt-miR529b ppt-miR529c ppt-miR529d

C.

2185799162 2183893560 ath-miR159a

ath-miR159b ath-miR159c osa-miR159c osa-miR159b osa-miR159d ath-miR319a ath-miR319b ath-miR319c osa-miR319a osa-miR319b

D.

2185809227 2185755300 2185768332 2185552337 2185477245 2185874405 2185853634 vi-miR477 ptc-miR477a ptc-miR477b ppt-miR477a-5p ppt-miR477b ppt-miR477c ppt-miR477d ppt-miR477e ppt-miR477h ppt-miR477f ppt-miR477g-5p
$T G A C A G A A G A G A G T G A G C A C \ldots$

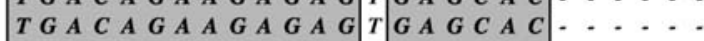
$T G A C A G A A$ G A $G A G G \mid G A G C A C$

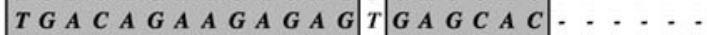

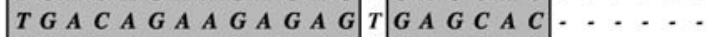

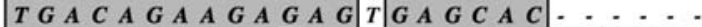
\begin{tabular}{llllllllll|lllllllllllllll}
$T$ & $G$ & $A$ & $C$ & $A$ & $G$ & $A$ & $A$ & $G$ & $A$ & $T$ & $A$ & $G$ & $A$ & $G$ & $A$ & $G$ & $C$ & $A$ & $C$ & $A$ & $G$ & $A$ & $T$ & $G$
\end{tabular}

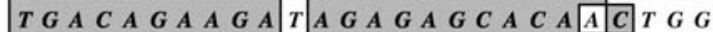
\begin{tabular}{llllllllll|llllllllllllll}
$T$ & $G$ & $A$ & $C$ & $A$ & $G$ & $A$ & $A$ & $G$ & $A$ & $T$ & $A$ & $G$ & $A$ & $G$ & $A$ & $G$ & $C$ & $A$ & $C$ & $A$ & $G$ & $A$ & $T$
\end{tabular}

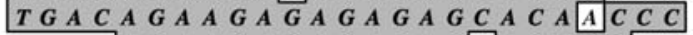

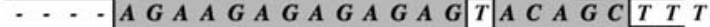

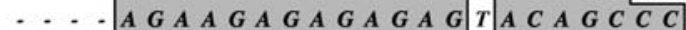

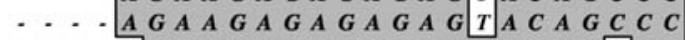

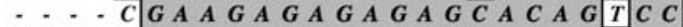
-. $C G A A G A G A G A G A G C A C A G C C C$ - - $C G A A A G A G A G A G A G C A C A A G C$

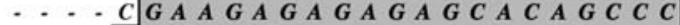
- - $A G A A$ G A G A G A G A G C A C A G C C C T $G$ A $C A G A A G A G A G A G A G C A C A G C C C$

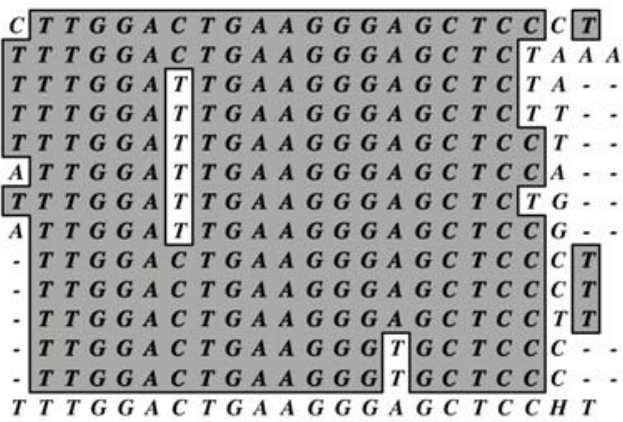

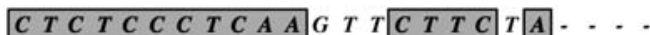

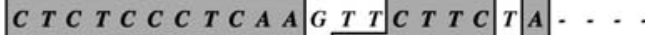

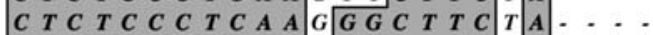

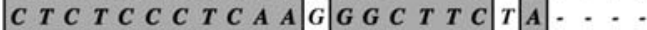

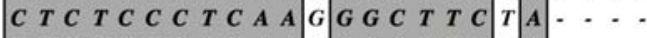

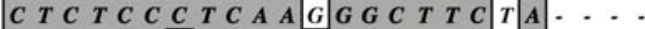

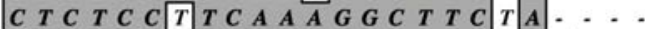
A $T$ T C T C C C C T C A A A G G C T T C C A A - A $\quad$ T C $C$ T C C C C $T$ C C A A $T$ T C $T$ T C C C $C$ T C A

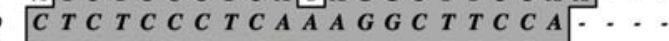
C $T$ T C T $C$ C C C $C$ T C A A A G G C T T C C A - . C $T$ T C C $T$ T C $T$ T C C C C $T$ C C $T$ T C C C

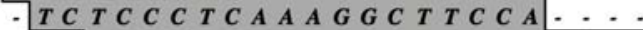
- $\quad$ T C C C T C A A A G G C T T C C A A C A A - $-T C C C T C A A A G G C T T C C A A C A A$ C TC TCC C TCA A A G G C T TC C A A C A A 

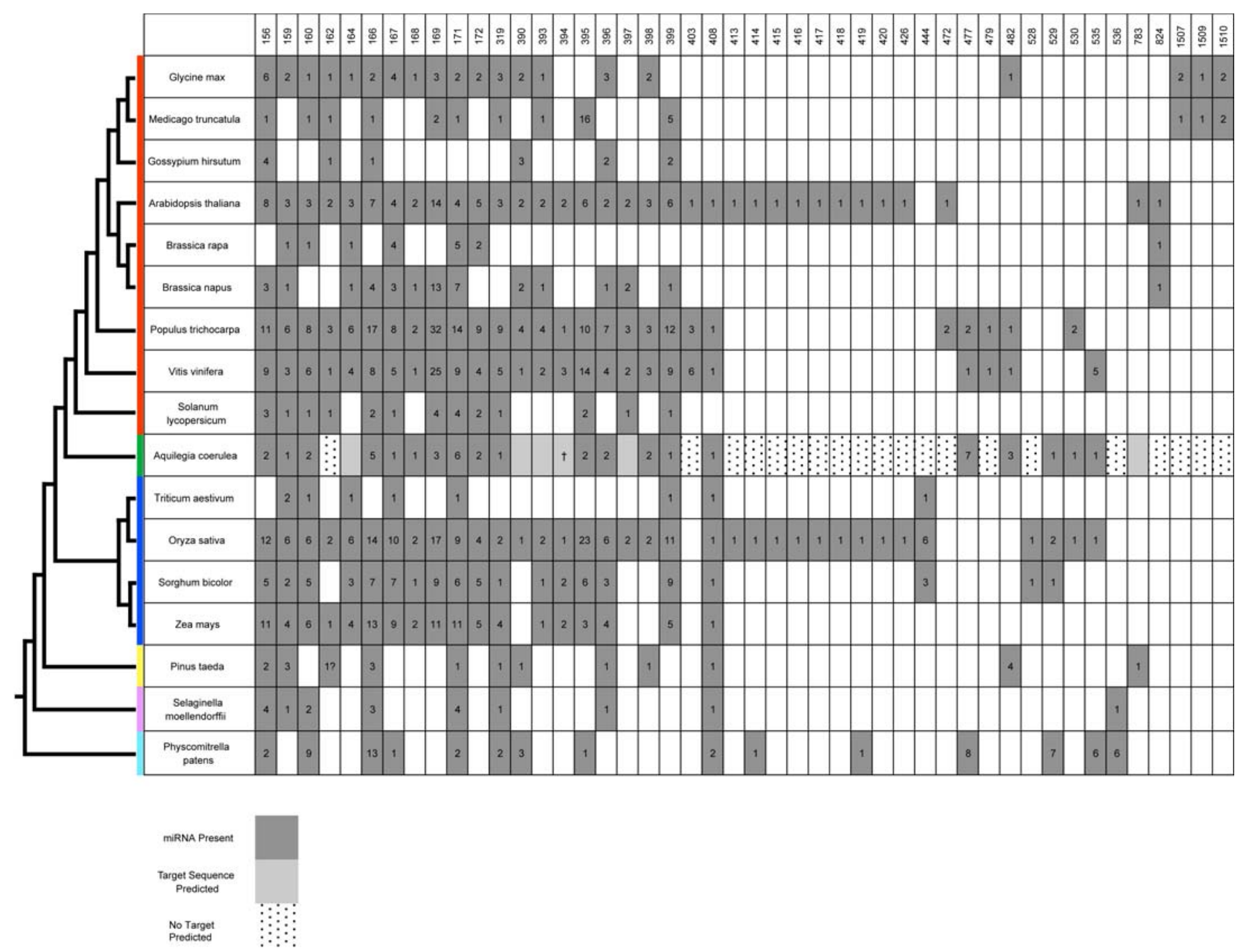
Supplementary Table 1

\begin{tabular}{|c|c|c|c|}
\hline microRNA family & aqc-miRNA gi & Mature & $\begin{array}{c}\text { Genbank Accession } \\
\text { Number }\end{array}$ \\
\hline aqc-miRNA156a & 2185892723 & TGACAGAAGATAGAGAGCAC & \\
\hline aqc-miRNA156b & 2185518132 & TGACAGAAGATAGAGAGCAC & \\
\hline aqc-miRNA159 & 2183893560 & TTTGGACTGAAGGGAGCTCTA & \\
\hline aqc-miRNA160a & 2185524891 & TGCCTGGCTCCCTGGATGCCA & \\
\hline aqc-miRNA160b & 2185745223 & TGCCTGGCTCCCTGTATGCCA & \\
\hline aqc-miRNA166a & 2185638590 & TCGGACCAGGCTTCATTCCTC & \\
\hline aqc-miRNA166b & 2185550821 & TCGGACCAGGCTTCATTCCCC & \\
\hline aqc-miRNA166c & 2185683719 & TCGGACCAGGCTTCATTCCT & \\
\hline aqc-miRNA166d & 2185830185 & TCGGACCAGGCTTCATTCCTC & \\
\hline aqc-miRNA166e & 2185503206 & TCGGACCAGGCTTCATTCCCC & \\
\hline aqc-miRNA167 & 2185494083 & TCAAGCTGCCAGCATGATCTA & \\
\hline aqc-miRNA168 & 2185845089 & TGGCTTAGTGCAGCTCGGGGA & \\
\hline aqc-miRNA169a & 2185604956 & TAGCCAAGGATGACTTGCCTA & \\
\hline aqc-miRNA169b & 2185687520 & TAGCCAAGGATGACTTGCCTG & \\
\hline aqc-miRNA169c & 2185493504 & CAGCCAAGGATGACTTGCCGG & \\
\hline aqc-miRNA171a & 2185568457 & TGATTGAGCCGTGCCAATATC & \\
\hline aqc-miRNA171b & 2185624710 & TGATTGAGCCGTGCCAATATC & \\
\hline aqc-miRNA171c & 2185689689 & TAATTGAACCGCACTAATATC & \\
\hline aqc-miRNA171d & 2185735537 & TGATTGAGCCGTGCCAATATC & \\
\hline aqc-miRNA171e & 2185815653 & TGAATGAACCGAGCCAACATC & \\
\hline aqc-miRNA171f & 2185468845 & TAATTGAGCCGTGCCAATATC & \\
\hline aqc-miRNA172a & 2185776282 & AGAATCTTGATGATGCTGCAT & \\
\hline aqc-miRNA172b & 2185788415 & GGAATCTTGATGATGCTGCAT & \\
\hline aqc-miRNA319 & 2185799162 & TTGGACTGAAGGGAGCTCCCT & \\
\hline aqc-miRNA395a & 2185894728 & CTGAAGGGTTTGGAGGAACTC & \\
\hline aqc-miRNA395b & 2185728750 & CTGAAGGGTTTGGAGGAACTC & \\
\hline aqc-miRNA396a & 2185513426 & TTCCACAGCTTTCTTGAACTG & \\
\hline aqc-miRNA396b & 2185617799 & TTCCACAGCTTTCTTGAACTT & \\
\hline
\end{tabular}




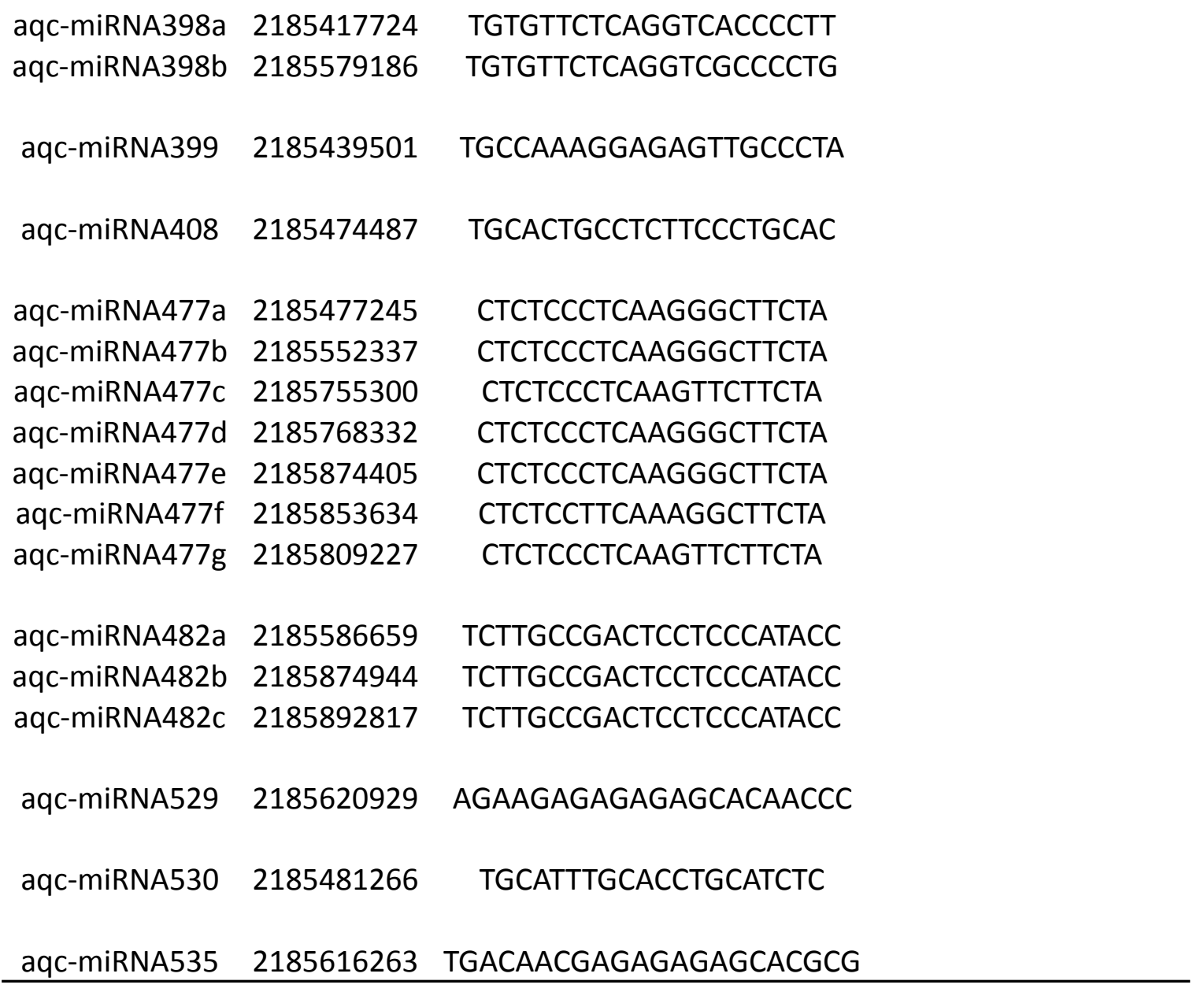

gi: gene index number corresponding to $A$. coerulea whole genome sequence databa: 
Supplementary Table 2

Potential targets of Aquilegia coerulea microRNAs

\begin{tabular}{|c|c|c|c|c|}
\hline microRNA family & Targeted Protein & Target Function & TC\# & Top Scoring A.t. locus \\
\hline \multirow[t]{7}{*}{156} & Squamosa promoter binding protein-like 2 (SBP) & Transcription factor & TC31380, TC24142 & AT5G43270 \\
\hline & Squamosa promoter binding protein-like 6 (SBP) & Transcription factor & $\begin{array}{l}\text { TC26823, TC21170, } \\
\text { TC21808, DR954092 }\end{array}$ & AT1G69170 \\
\hline & Squamosa promoter binding protein-like 13 (SBP) & Transcription factor & TC32167, DR949672 & AT5G50570 \\
\hline & Squamosa promoter binding protein-like 14 (SBP) & Transcription factor & TC21707 & AT1G20980 \\
\hline & Squamosa promoter binding protein-like 12 (SBP) & Transcription factor & TC31356 & AT3G60030 \\
\hline & Growth Regulating Factor 2 (GRF2) & Transcription factor & TC25281 & AT4G37740 \\
\hline & Unknown & Unknown & TC31827 & AT1G40133 \\
\hline 159 & None & & & \\
\hline \multirow[t]{2}{*}{160} & Transposable element & & DT734946 & AT5G28526 \\
\hline & Psuedo-response regulator 2 (APRR2) & Transcription factor & TC22019 & AT4G18020 \\
\hline 162 & None from EST database & & None from EST database & \\
\hline \multirow[t]{5}{*}{164} & Cup-Shaped Cotyledon 2 (CUC2) & Transcription factor & DR949423, TC27953 & AT5G53950 \\
\hline & NAC Domain containing protein & Transcription factor & TC27854 & AT5G39610 \\
\hline & $\begin{array}{l}\text { Leucine-rich repeat transmembrane protein } \\
\text { kinase }\end{array}$ & Metabolism & DT731408 & AT3G28040 \\
\hline & UDP-glucose pyrophosphorylase & Stress & TC21677 & AT3G03250 \\
\hline & 4-coumarate-CoA ligase family protein & Stress & DT741735, TC24498 & AT1G20510 \\
\hline 166 & Class III HD-Zip protein & Transcription factor & TC30761 & AT1G52150 \\
\hline 167 & Auxin response factor 6 & Transcription factor & TC29952, TC24356 & AT1G30330 \\
\hline \multirow[t]{2}{*}{168} & Unknown & Metabolism & DT747736, DT74773 & AT3G48870 \\
\hline & Anion Transporter 2 & Transport & TC29087 & AT4G00370 \\
\hline \multirow[t]{2}{*}{169} & CCAAT-binding factor (HAP2) & Transcription factor & DR924236 & AT1G72830 \\
\hline & Lipase class 3 family protein & Metabolsim & TC31138, DR927955 & AT5G18640 \\
\hline \multirow[t]{2}{*}{$170 / 171$} & Scarecrow-like protein & Transcription factor & TC21487, TC28359, TC25185 & AT2G45160 \\
\hline & Arabidopsis Pumilio 2 (APUM2)-like & RNA binding & TC29397 & AT2G29190 \\
\hline \multirow[t]{10}{*}{172} & APETALA2 & Transcription factor & $\begin{array}{l}\text { DT732637, TC28864, } \\
\text { TC22964, TC24245 }\end{array}$ & AT4G36920 \\
\hline & Target of EAT 1 (TOE1) & Transcription factor & $\begin{array}{l}\text { DT741192, TC28449, } \\
\text { TC32032 }\end{array}$ & AT2G28550 \\
\hline & Unknown & Unknown & DR954144 & AT5G65290 \\
\hline & Unknown & $\begin{array}{l}\text { protein binding, zinc ion } \\
\text { binding }\end{array}$ & TC26960 & AT3G48070 \\
\hline & Spike1 (SPK1) & Metabolism & DT764297 & AT4G16340 \\
\hline & SC35-like splicing factor & Metabolism & TC22146 & AT3G13570 \\
\hline & Unknown & Transcription factor & DT749684 & AT3G10070 \\
\hline & Ubitiquitin-conjugating enzyme & Metabolism & TC25860, TC21155 & AT5G05080 \\
\hline & F-box protein & Photoprocesses & TC28027 & AT4G02440 \\
\hline & Selenium binding protein & Stess & TC29593 & AT2G24440 \\
\hline 319 & $\begin{array}{l}\text { Diminuto 1, Enhanced Very-Low-Fluence } \\
\text { Responses } 1\end{array}$ & Photoprocesses & TC25461, DR922754 & AT3G19820 \\
\hline 390 & Cation symporter & Transport & TC30528 & AT5G03560 \\
\hline \multirow[t]{2}{*}{393} & Auxing Signalizng F-BOX 2 & Auxin & TC29517 & AT3G26810 \\
\hline & Transport Inhibitor Response 1 (TIR1) & Auxin & $\begin{array}{l}\text { TC20718, TC25434, } \\
\text { DR913737 }\end{array}$ & AT3G62980 \\
\hline 394 & F-box protein & Unknown & TC24293, TC26091 & AT1G27340 \\
\hline \multirow[t]{4}{*}{395} & Kinase & Metabolism & TC32489 & AT4G00955 \\
\hline & Unknown & Unknown & TC20934 & AT3G49590 \\
\hline & ATP sulfurylase & Stress & TC21492 & AT3G22890 \\
\hline & Unknown & Unknown & TC30052 & AT2G02370 \\
\hline \multirow[t]{3}{*}{396} & Unknown & Unknown & DR926810 & AT2G16760 \\
\hline & Calmodulin-binding protein & Stress & TC29396 & AT3G16940 \\
\hline & Mildew Resistance Locus & Stress & TC32094 & AT1G11000 \\
\hline
\end{tabular}


1-aminocyclopropane-1-carboxylate oxidase,

Ethylene forming enzyme

UDP-glucosyl transferase family protein

Laccase

Yip1 family protein

Metabolism

TC26926

AT5G01190

Metabolism

TC26200

AT2G38080

Unknown

TC24152

AT2G39805

Unknown

Unknown

DT728841

AT5G53740

None

beta-fructofuranosidase $A B A$ receptor

Metabolism

TC28894

AT1G56560

Metabolism

TC20517

AT2G20770

Xyloglucan Endotransglycosylase 6

Metabolism

TC30505

AT4G25810

Plastidic glucose-6-phosphate dehydrogenase

Metabolism

TC30732

TC20303

AT5G35790

Elongation Factor 1B-gamm

Metabolism

TC24529

AT1G09640

AT5G61330

None

Squamosa promoter binding protein-like 6 (SBP)

Transcription factor

DR954092, TC26823,

AT1G69170

Squamosa promoter binding protein-like 13 (SBP)

Osephin family protein

Transcription factor

Unknown

Squamosa promoter binding protein-like 14 (SBP)

Squamosa promoter binding protein-like 2 (SBP)

Threonine endopeptidase

FAD-binding domain-containing protein

Diacylglycerol kinase gene family

Transcription factor

Transcription factor

Unknown

Metabolism

Stress

Metal Tolerance Protein A2

Stress

1808, TC21170

DR949672, TC32167

AT5G50570

TC20719

AT3G54130

TC21707

TC31380, TC24142

AT1G20980

AT5G43270

TC32968

DR933604

AT4G01150

AT4G20800

TC29483

AT5G63770

DT745966, TC24319

AT3G58810

Metabolism

TC23269

AT2G35780

None

Phosphoenolpyruvate carboxylase

Methionine gamma-lyase

Metabolism

Metabolism

Glycoside hydrolase family protein

Carrier family protein

Metabolism

Transport

TC32408, TC27593

UDP-glucuronic acid decarboxylase

DT65875

TC33228

TC27947
AT3G14940

AT1G64660

AT5G43710

AT4G27940

AT3G53520 
A.

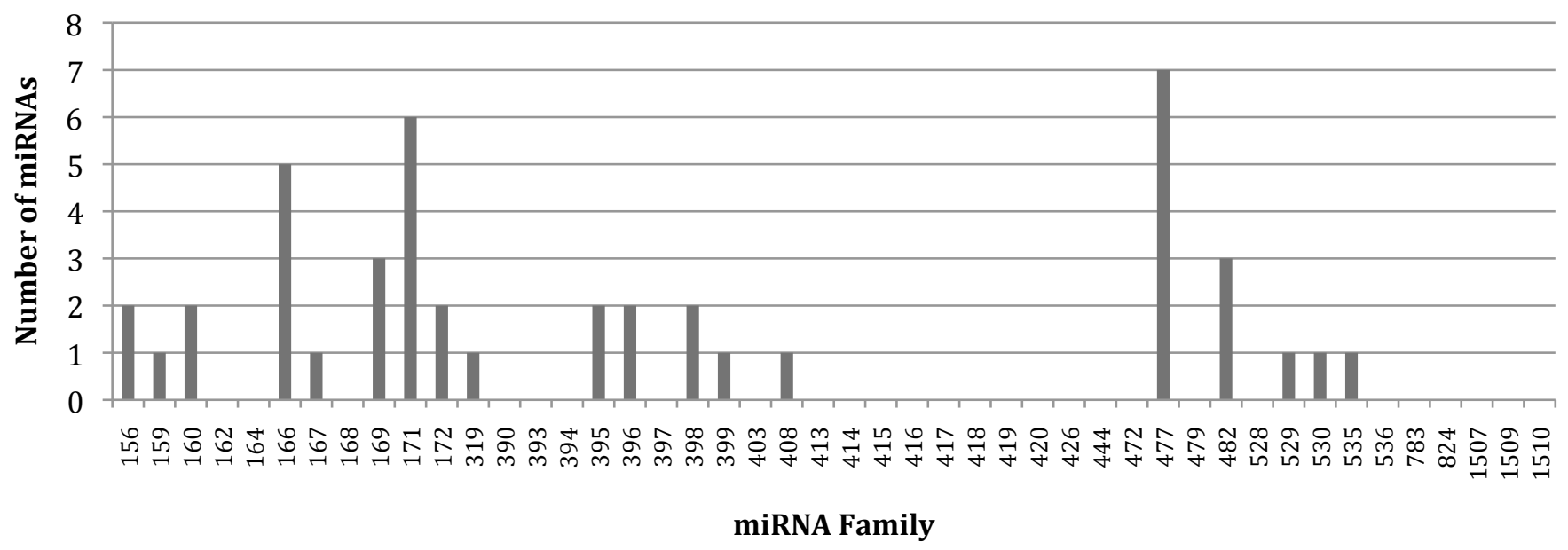

B.

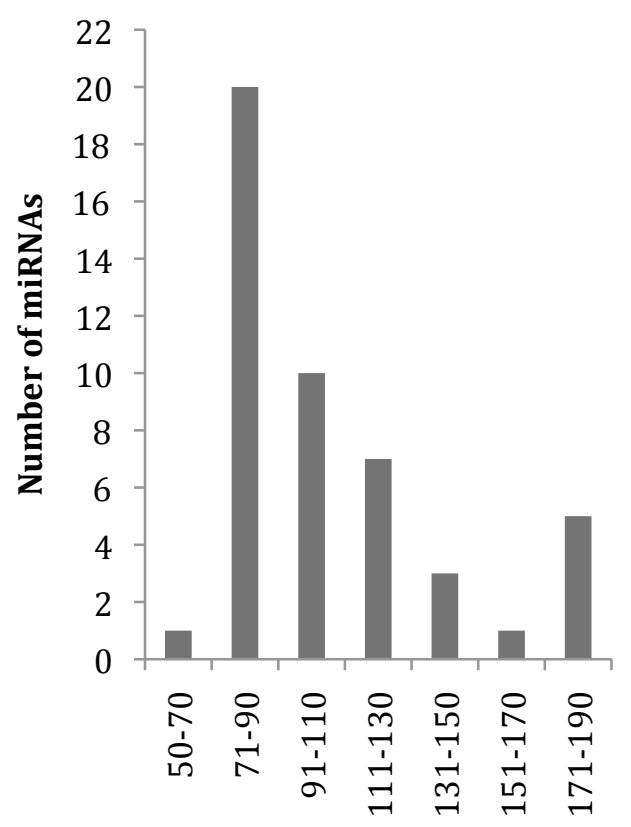

Sequence Length (nt)

Supplemental Figure 1

A. Size of miRNA families identified in in Aquilegia coerulea

B. Size distribution of Aquilegia coerulea miRNA predicted precursors 


\section{microRNA156}

2185892723mature
2185518132mature
DR949672binding
DR954092binding
TC21170binding
TC21707binding
TC21808binding
TC24142binding
TC26823binding
TC31356binding
TC31380binding
TC31827binding
TC32167binding
TC25281 binding

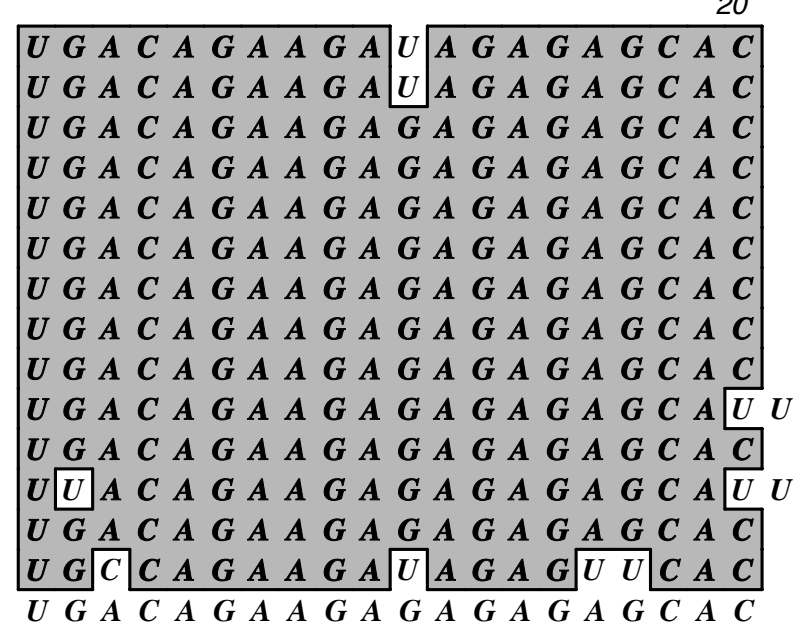

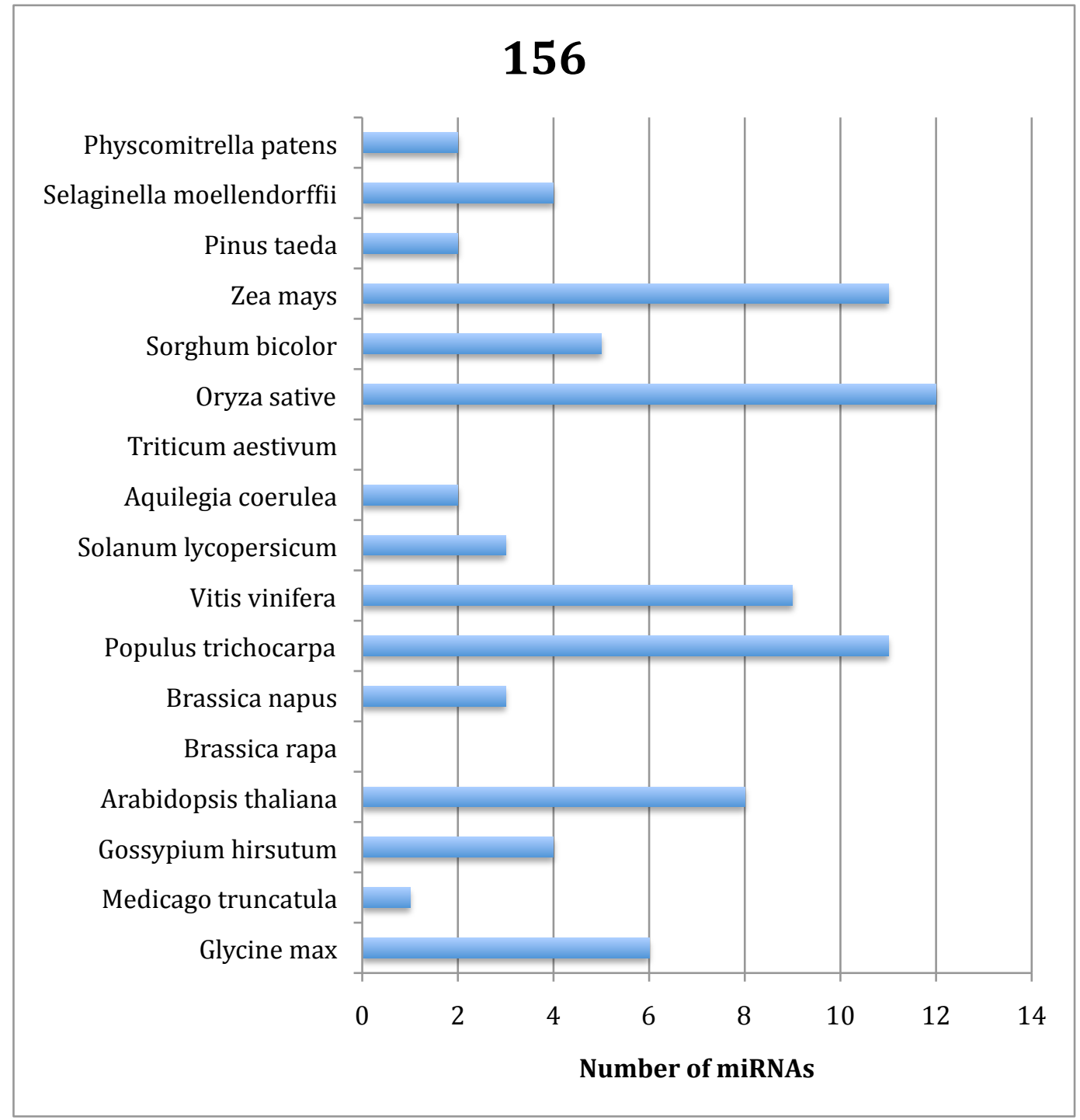

microRNA156 
microRNA156

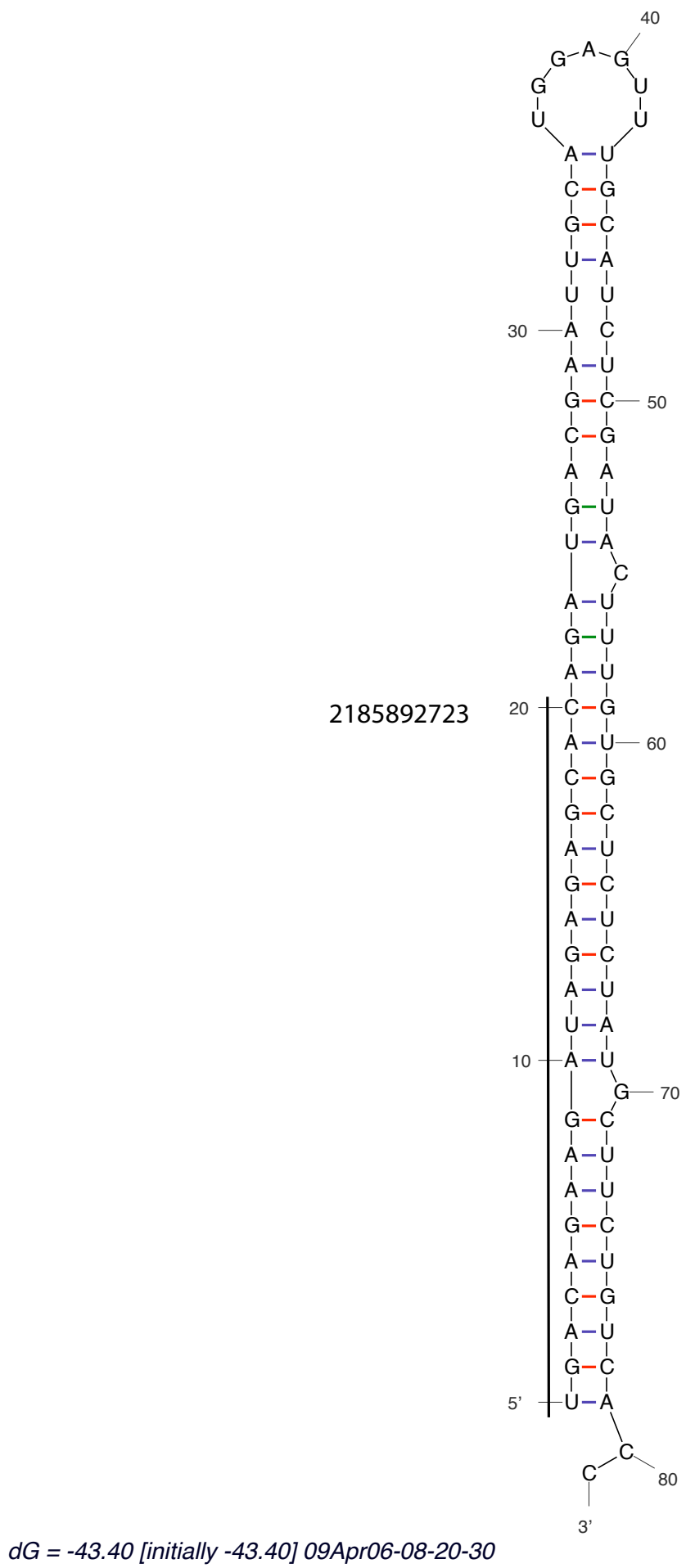


microRNA156

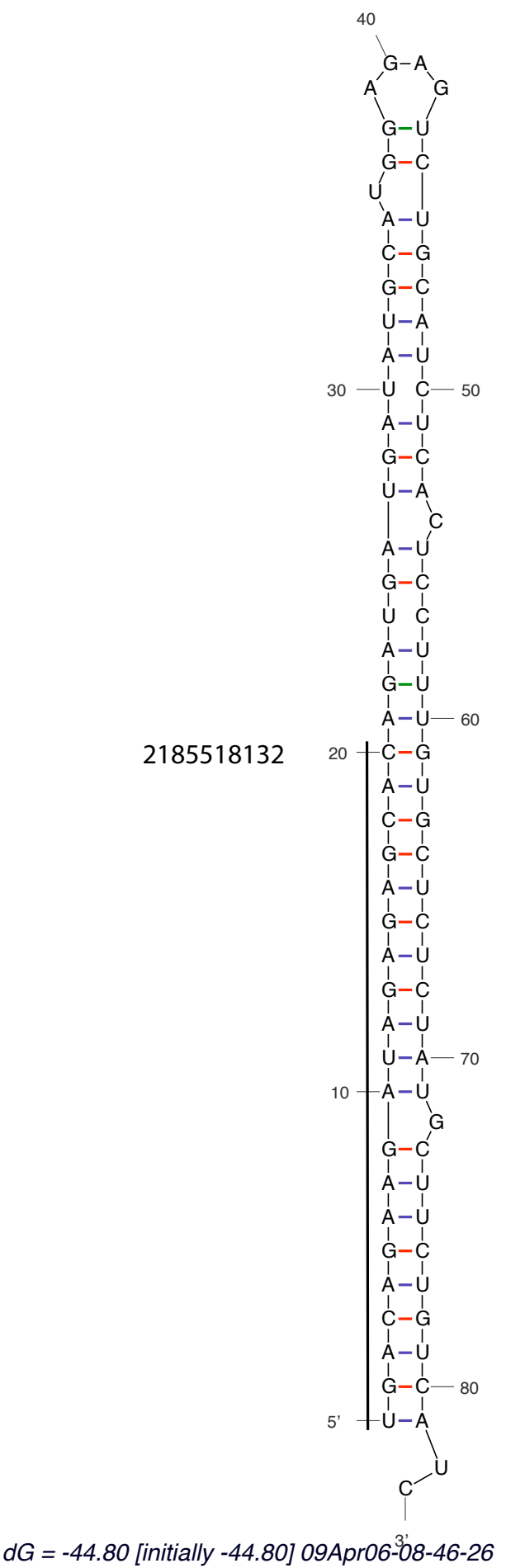

microRNA156 
microRNA159

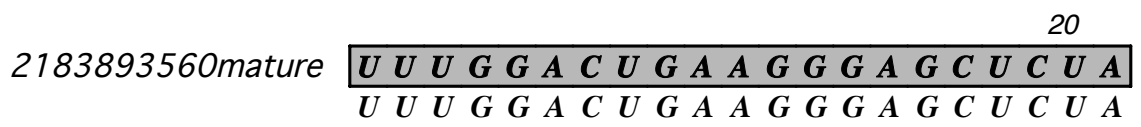

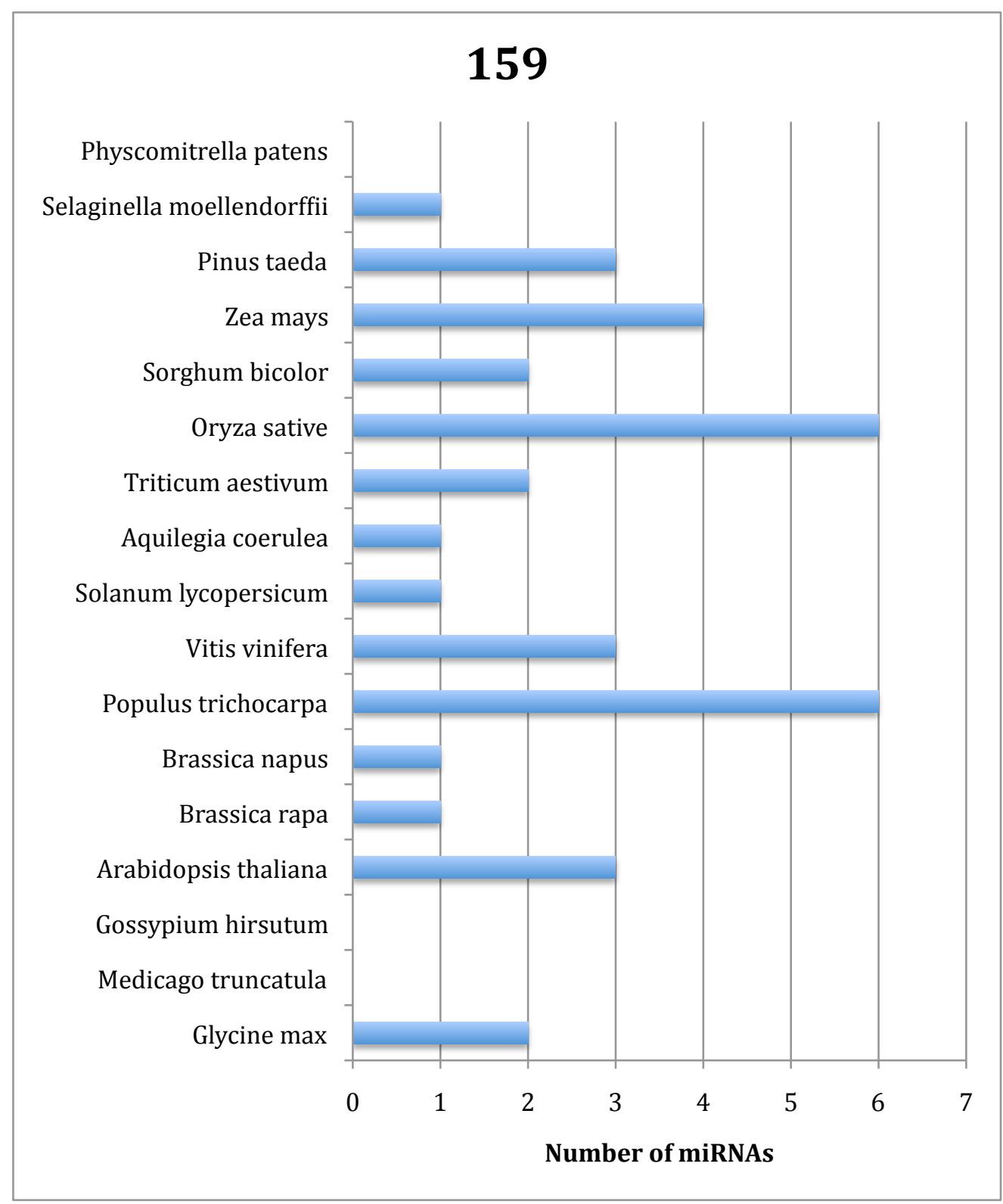


microRNA159

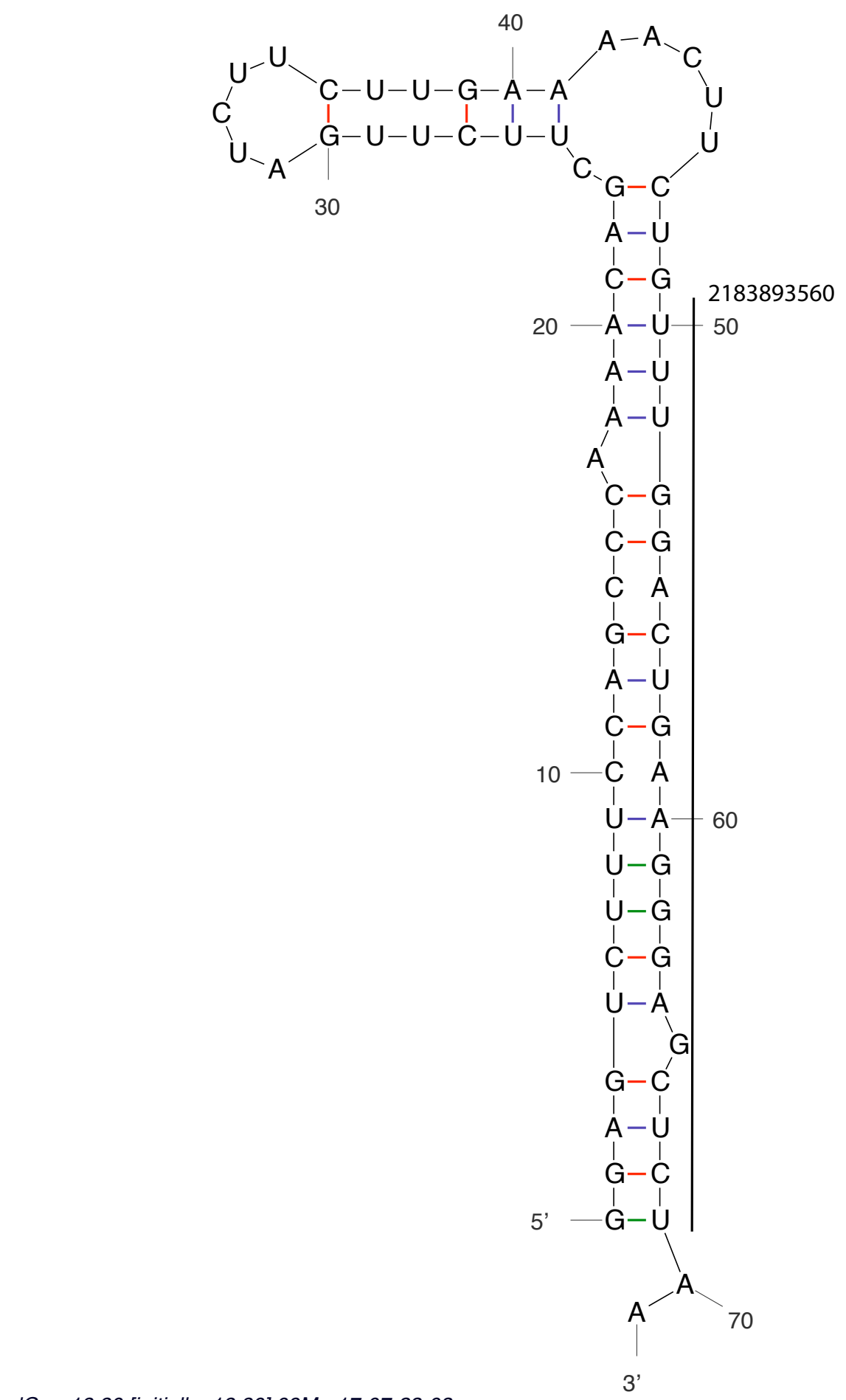

$d G=-18.80$ [initially -18.80 ] 09Mar17-07-23-03 
microRNA160

2185745223 mature

2185524891 mature

TC22019binding

EST1 168796 DT734946binding
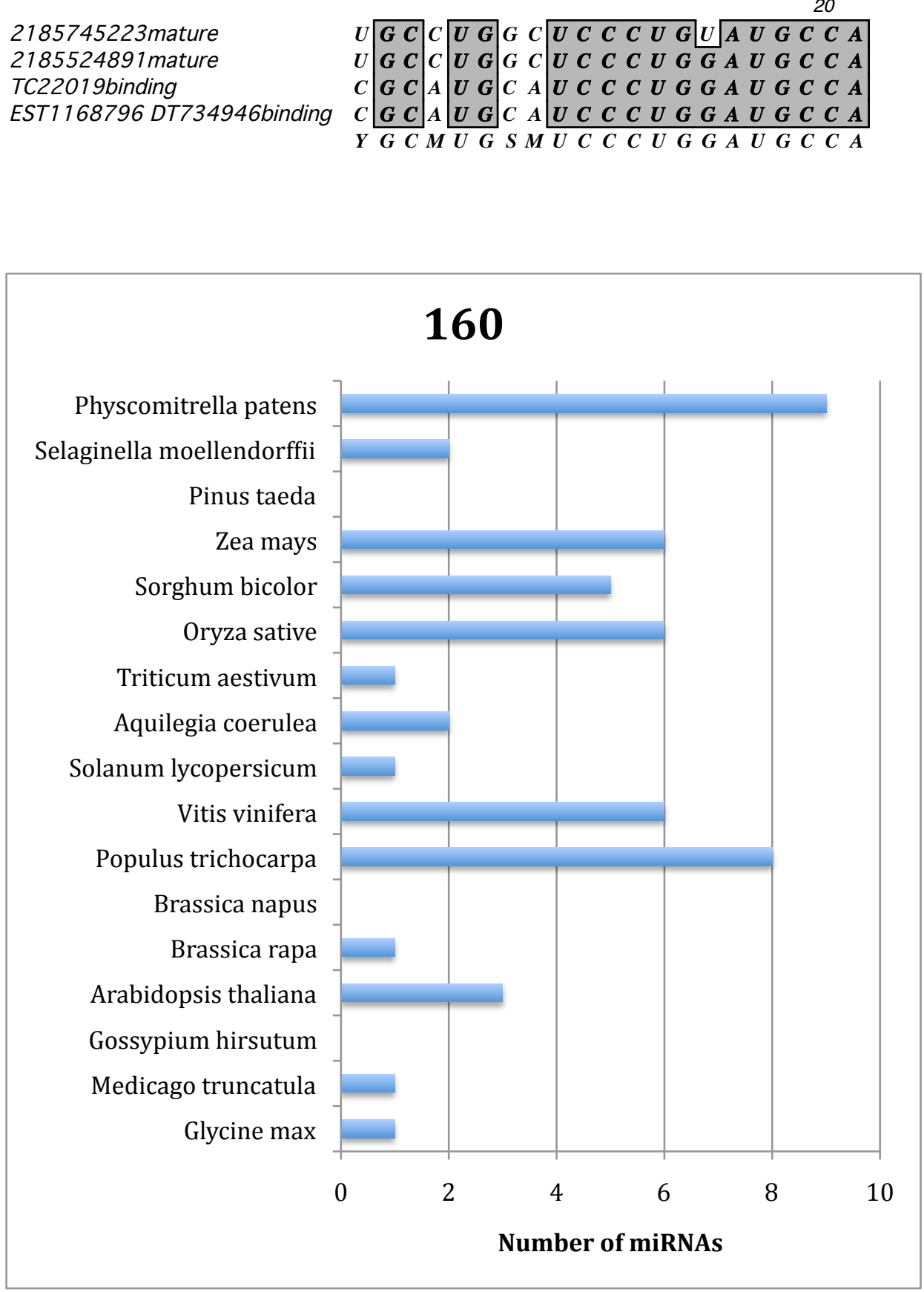
microRNA160

Output of sir_graph (ㅇ)

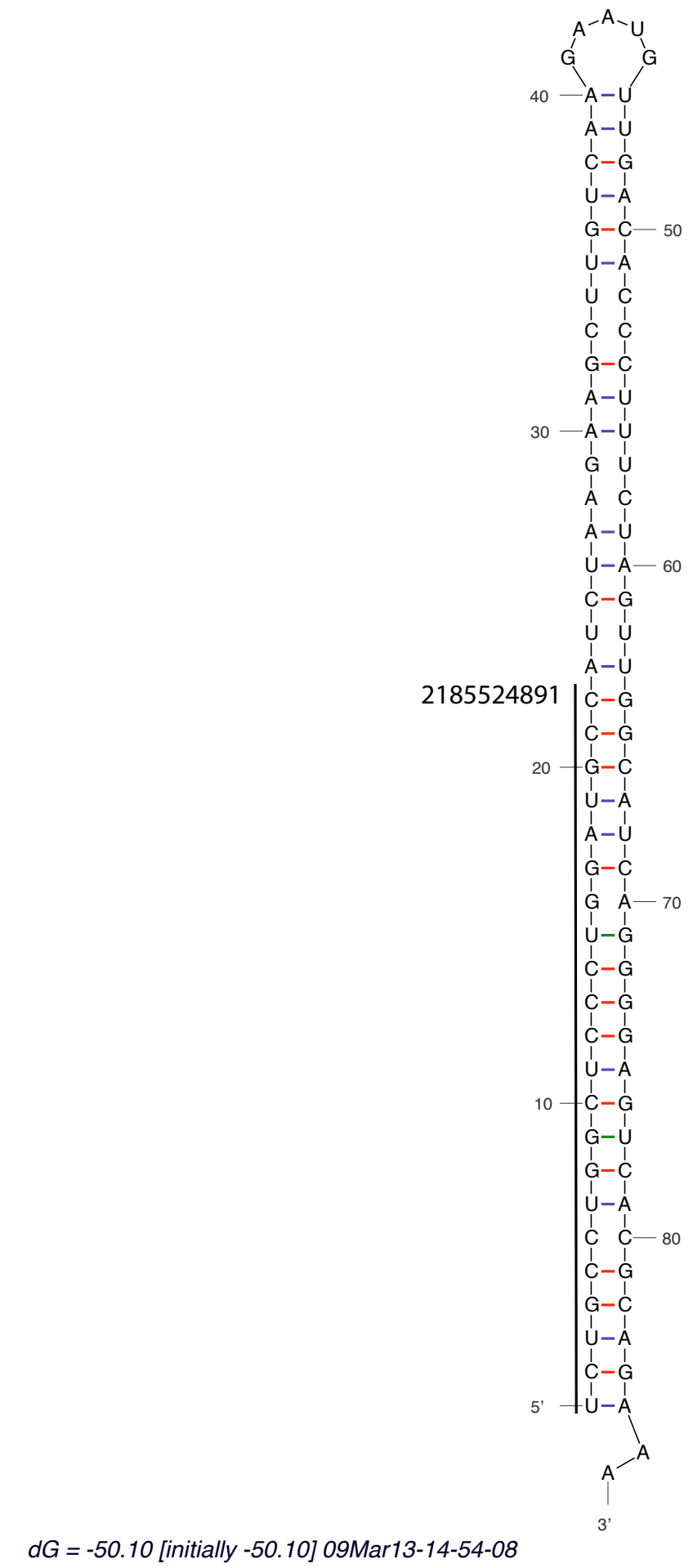

microRNA160 
microRNA160

Output of sir_graph (ब)
mfold 3.4

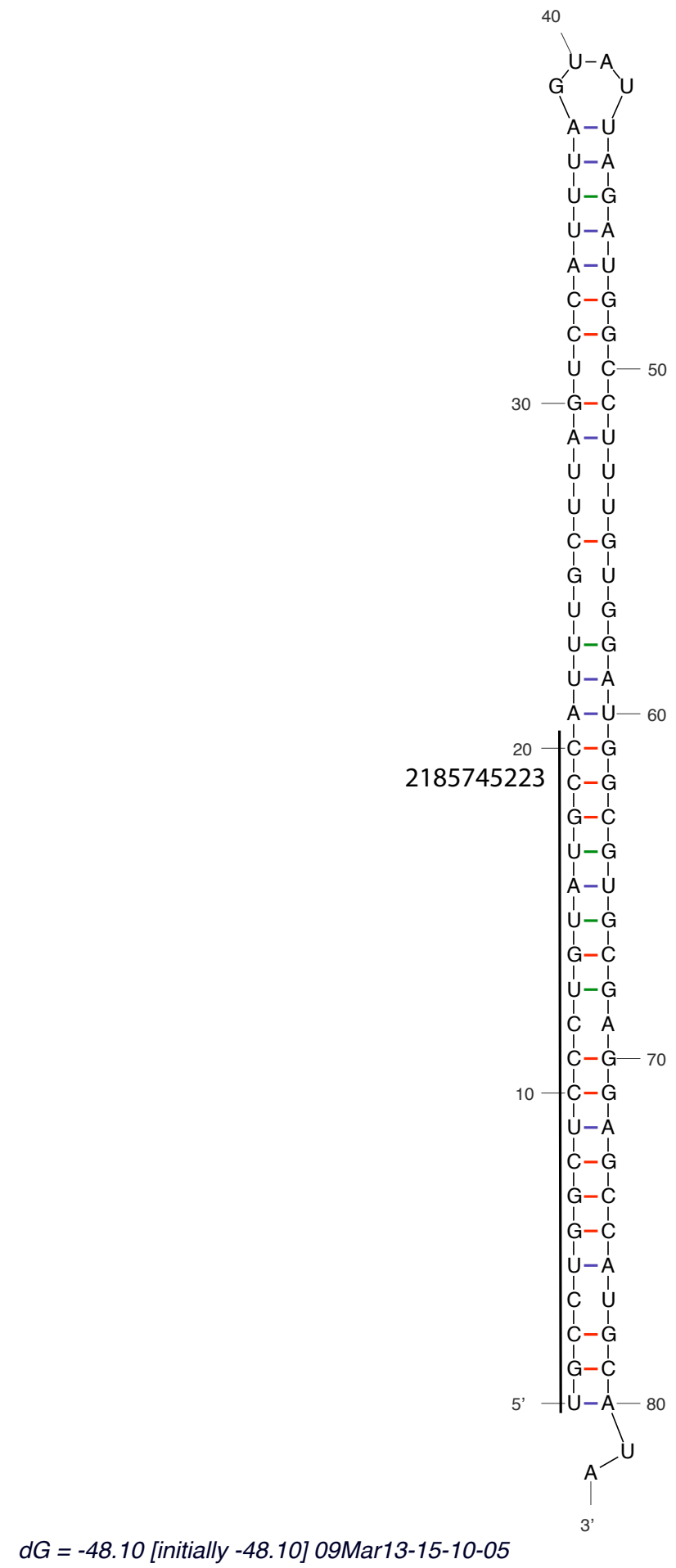

microRNA160 
microRNA164
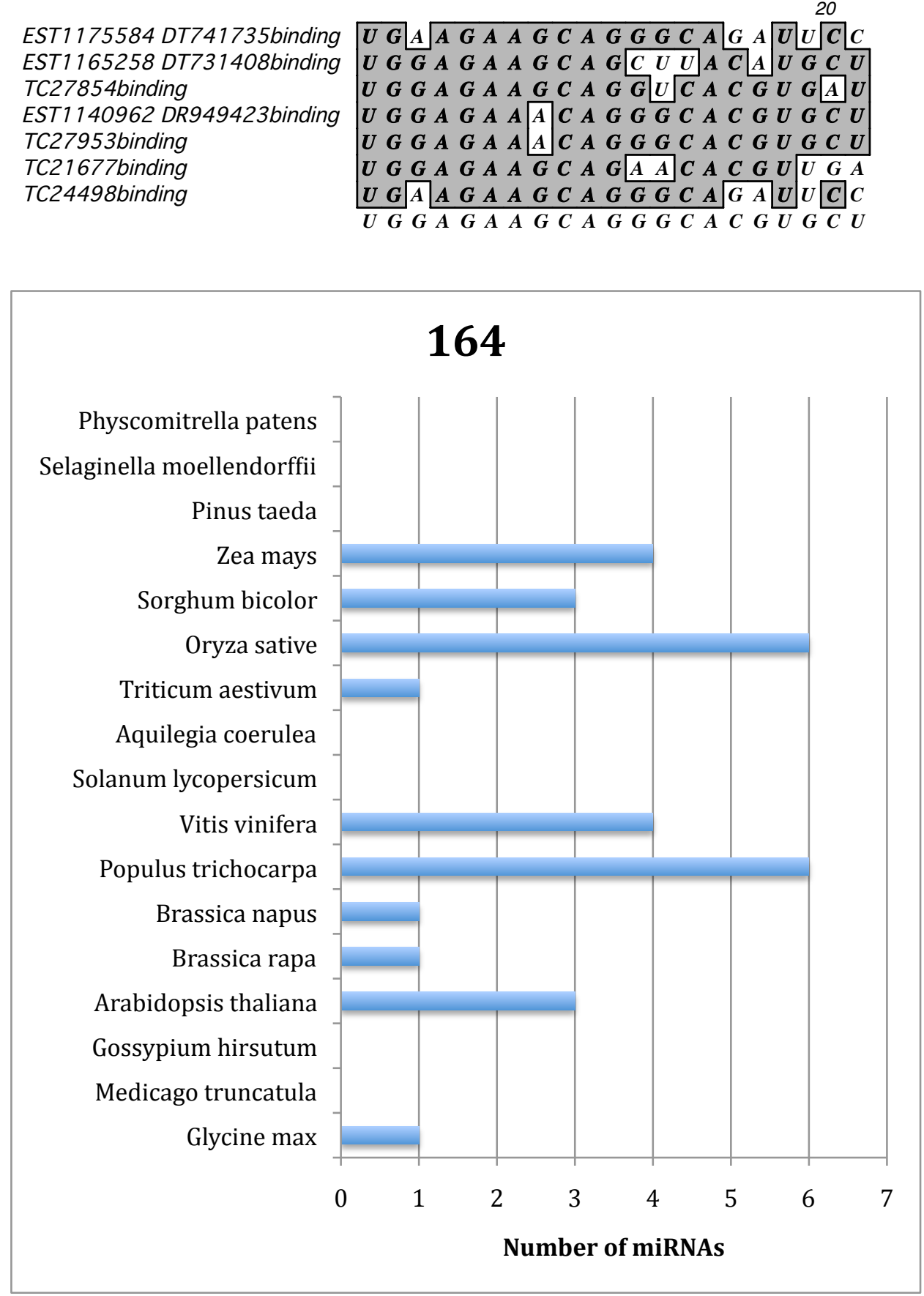
microRNA166

2185503206 mature 2185638590 mature gnlltil2185550821 mature gnlltil2185683719mature gnlltil2185830185mature TC30761binding
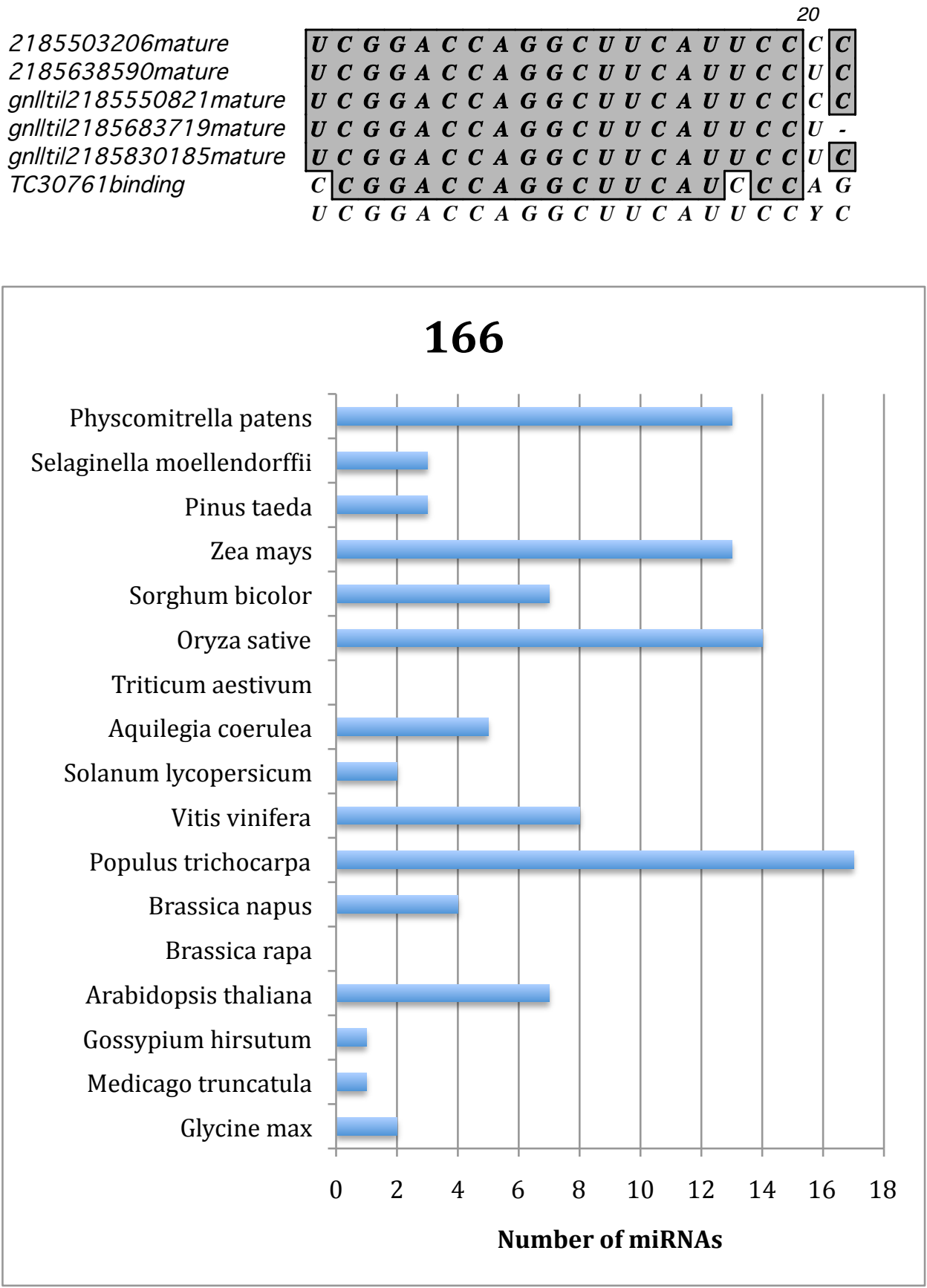
microRNA166

Output of sir_graph (बi)
mfold 3.4

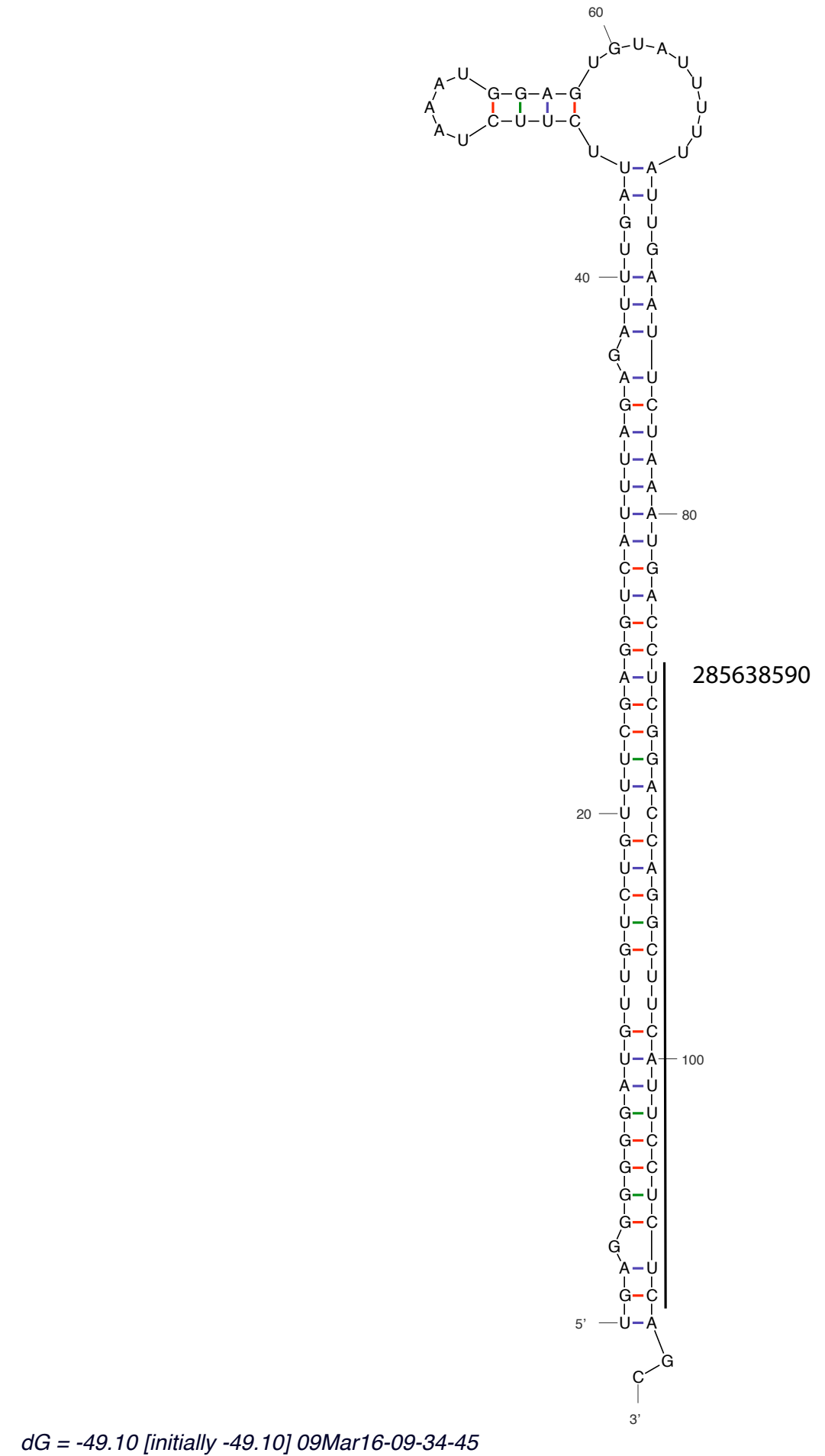

microRNA166 
microRNA166

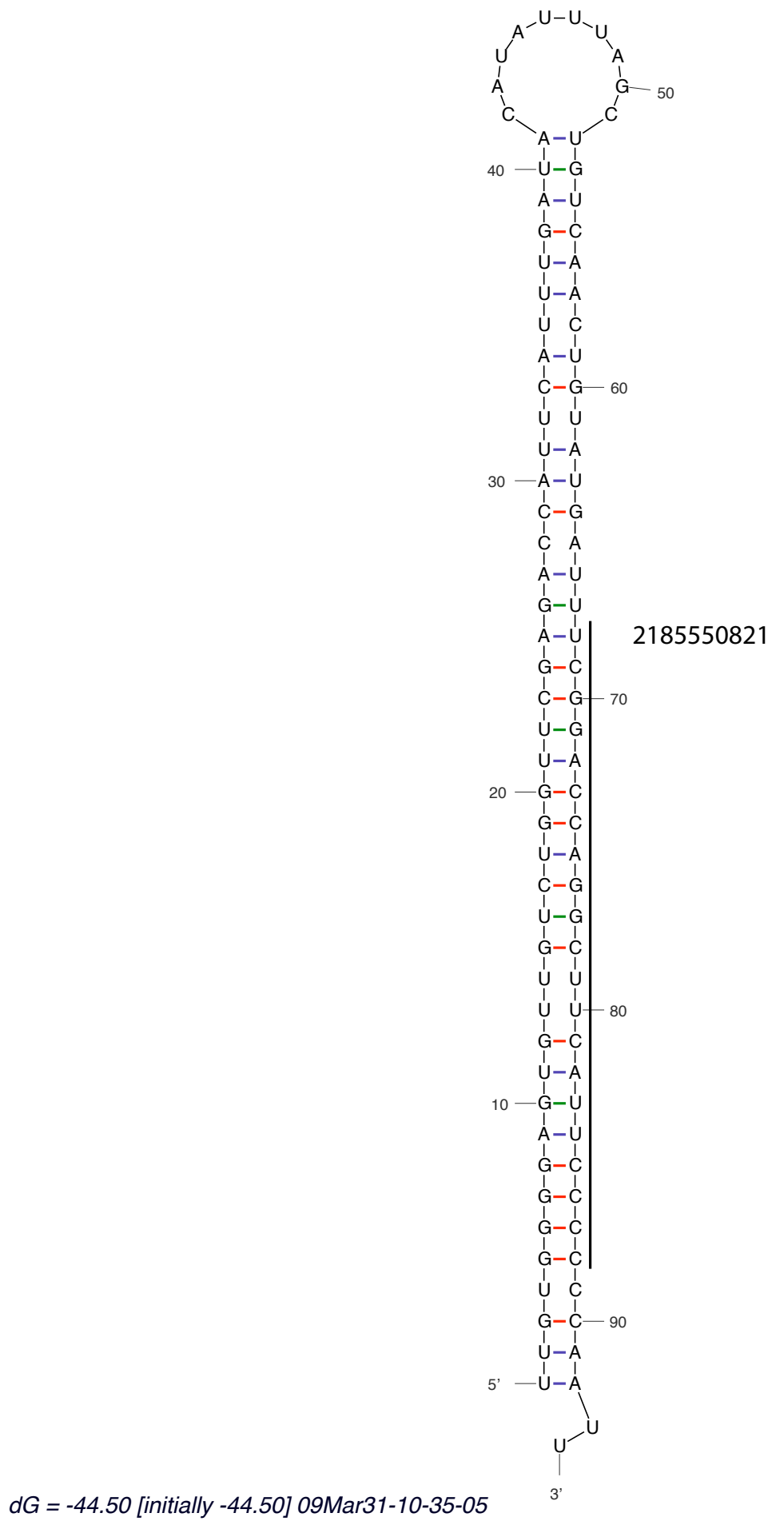


microRNA166

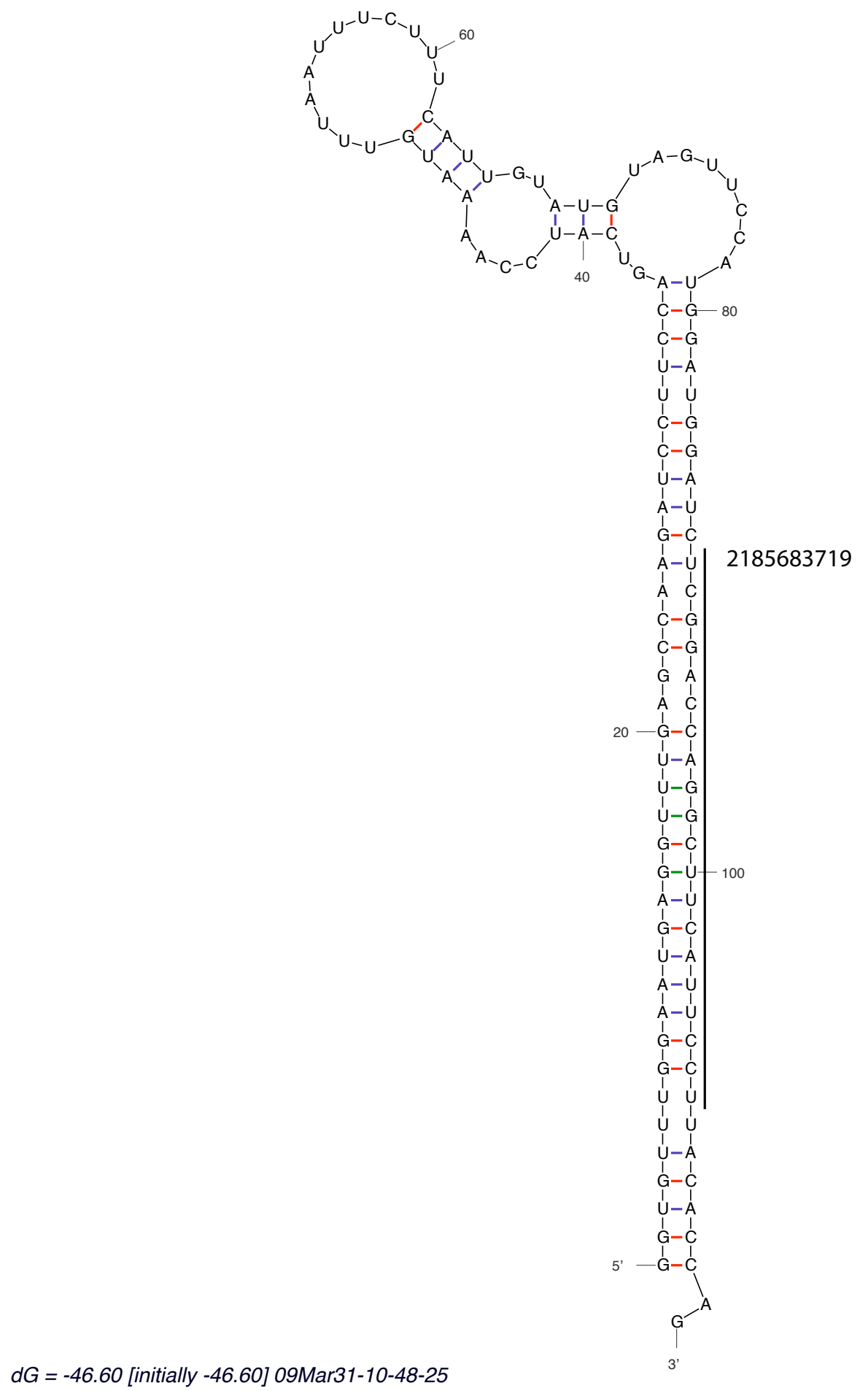


microRNA166

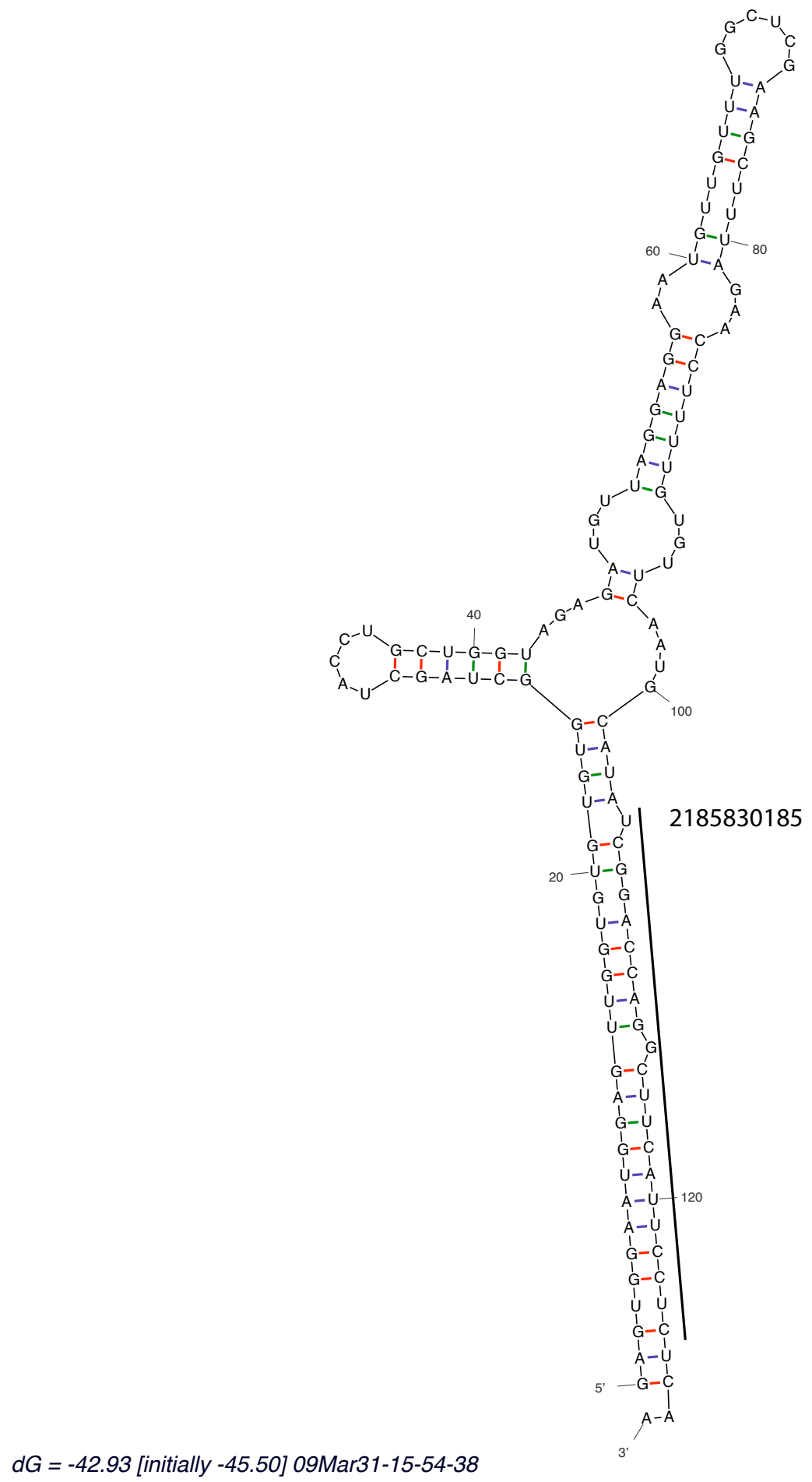


microRNA166

Output of sir_graph (@)
mfold 3.4

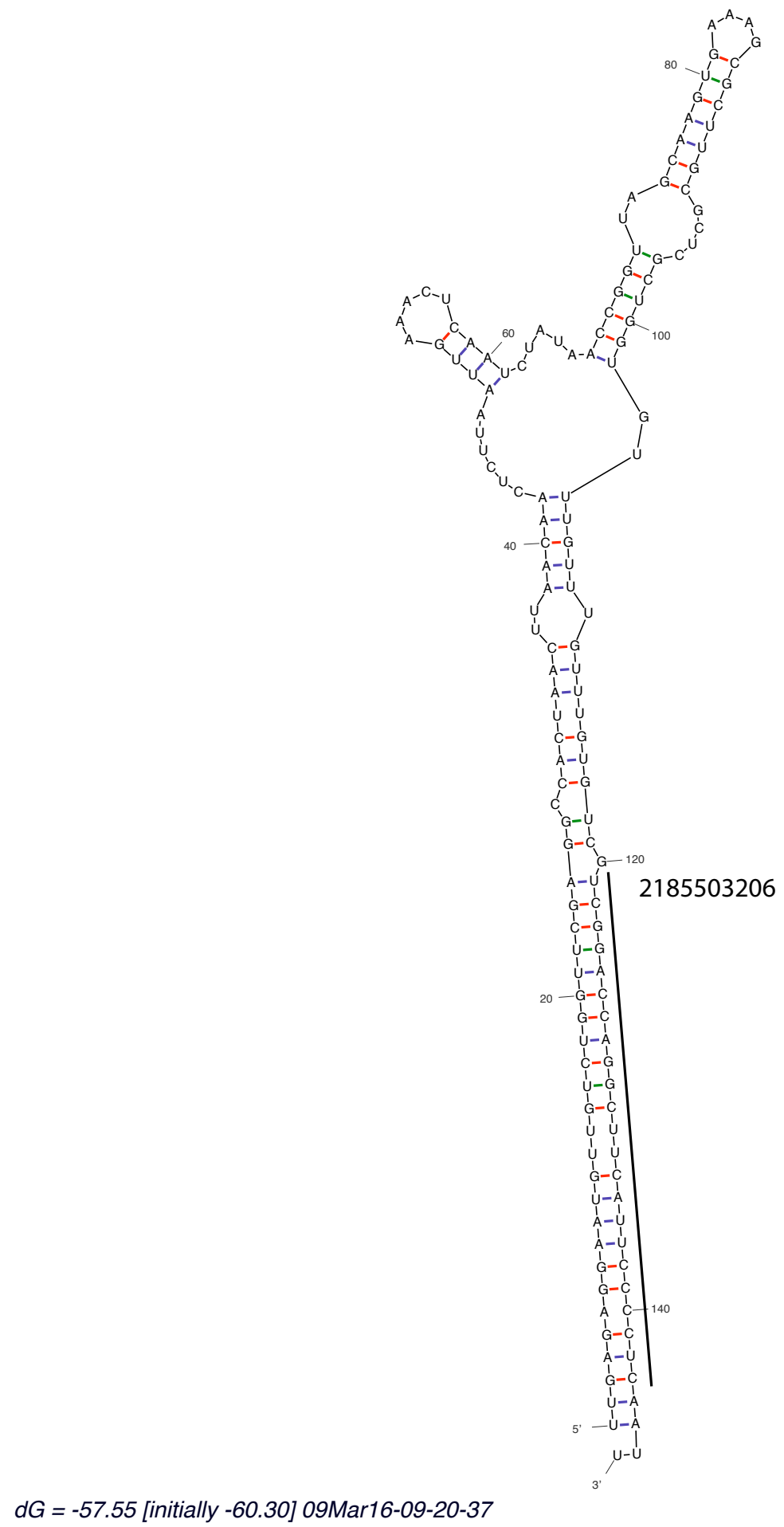

microRNA166 
microRNA167

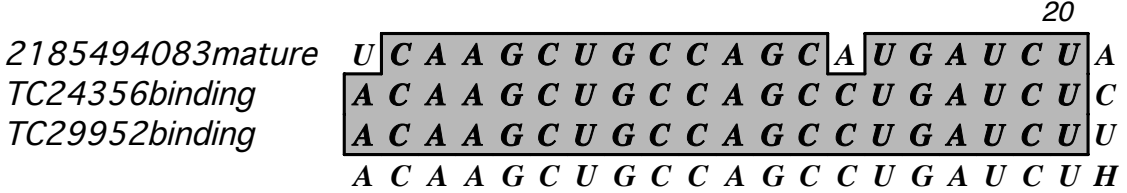

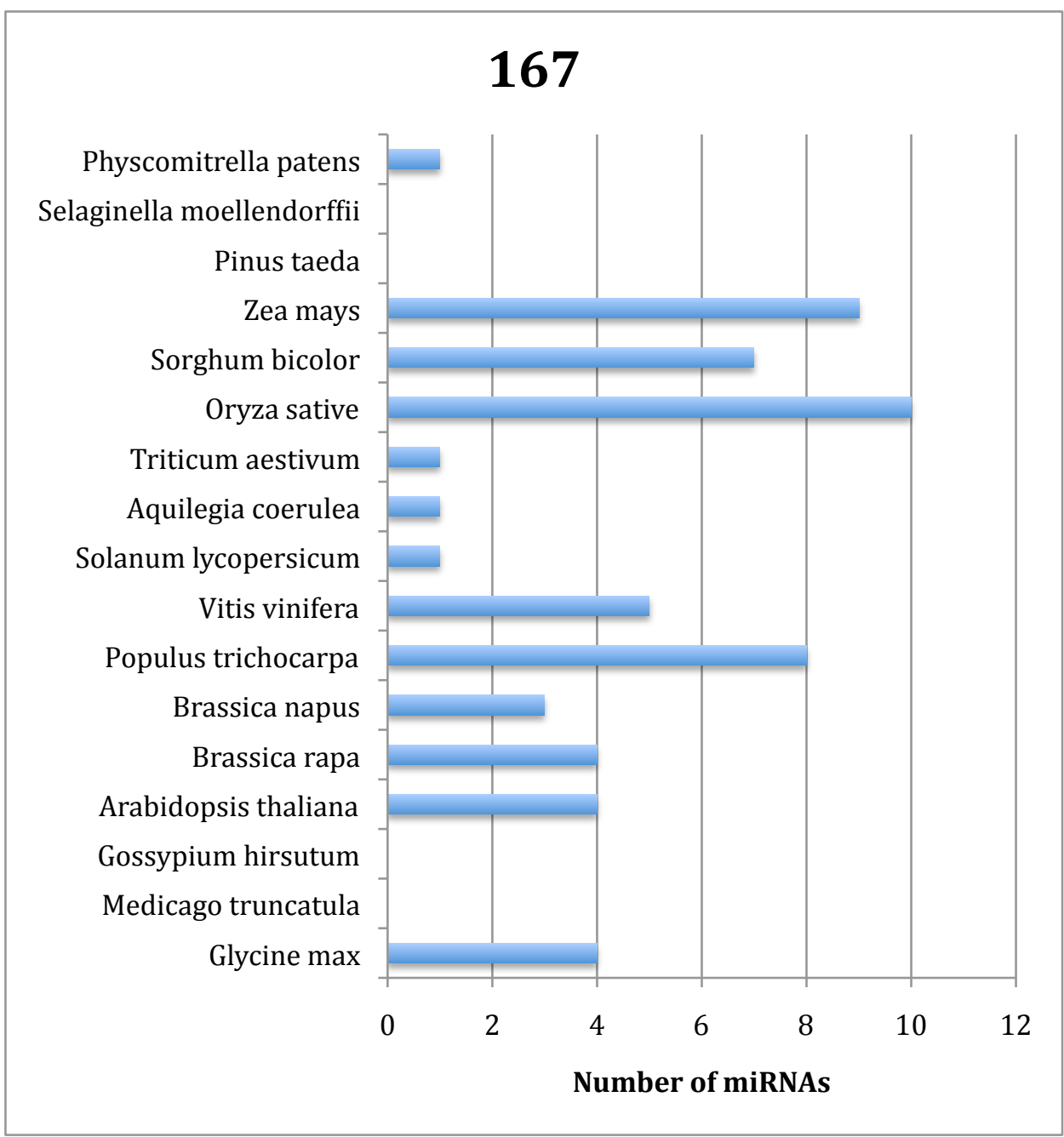


microRNA167

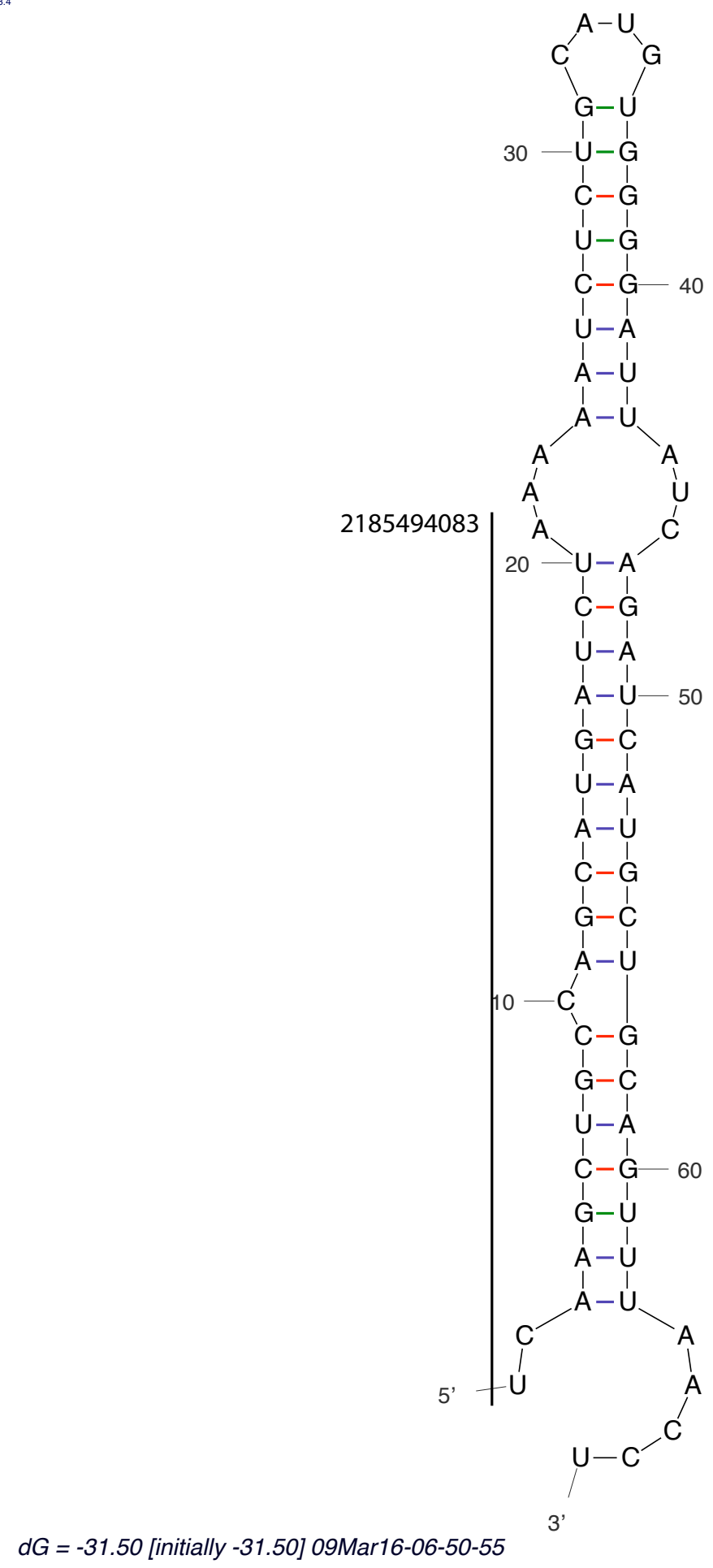


microRNA168

\begin{tabular}{|c|c|}
\hline $\begin{array}{l}\text { 2185845089mature } \\
\text { DT747736binding } \\
\text { TC29087binding }\end{array}$ & \begin{tabular}{|lllllllllllllllllllllll}
$U$ & $G$ & $G$ & $C$ & $U$ & $U$ & $A$ & $G$ & $U$ & $G$ & $C$ & $A$ & $G$ & $C$ & $U$ & $C$ & $G$ & $G$ & $G$ & $G$ & $A$ \\
$U$ & $G$ & $G$ & $C$ & $U$ & $U$ & $G$ & $G$ & $U$ & $G$ & $C$ & $A$ & $G$ & $C$ & $U$ & $C$ & $G$ & $G$ & $G$ & $G$ & $A$ \\
$U$ & $G$ & $G$ & $U$ & $U$ & $U$ & $G$ & $G$ & $C$ & $U$ & $C$ & $A$ & $G$ & $C$ & $U$ & $C$ & $G$ & $G$ & $G$ & $G$ & $A$ \\
\end{tabular} \\
\hline
\end{tabular}

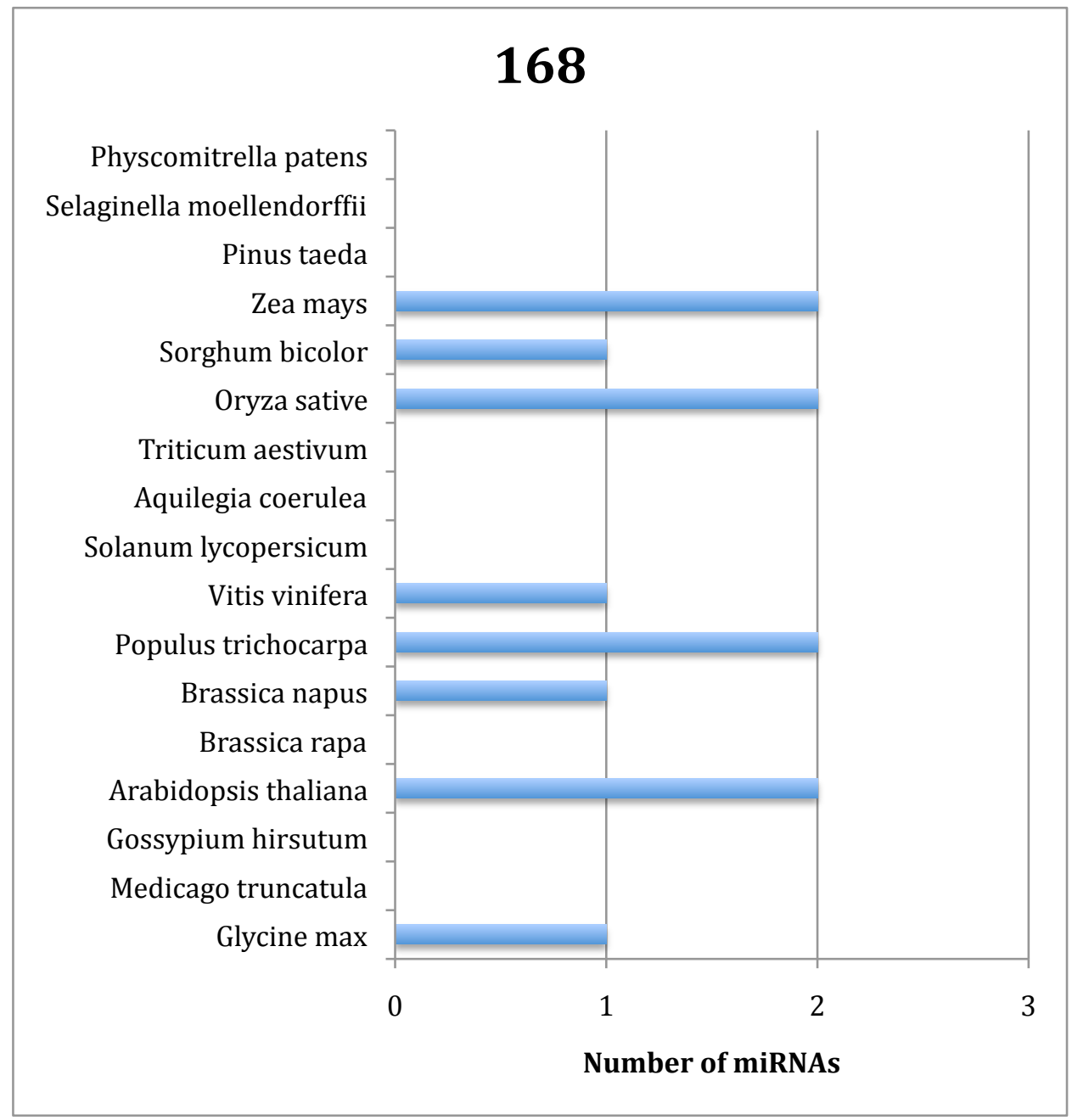


microRNA168

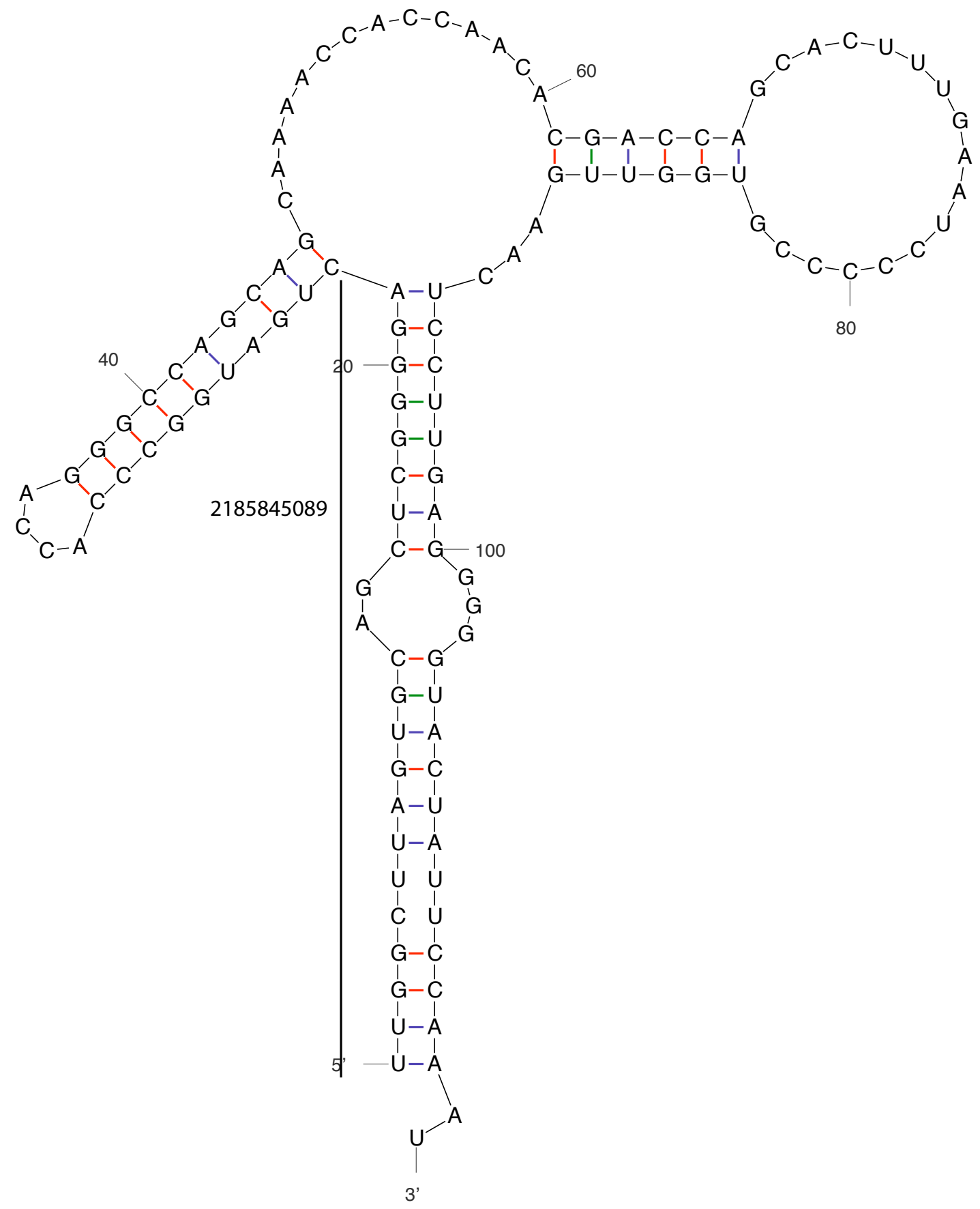

$d G=-40.45$ [initially -42.70 ] 09Apr01-09-19-45 
microRNA169

2185493504 mature

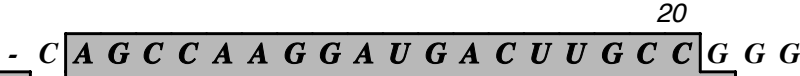
gnlltil2 185604956mature $\boldsymbol{G}$ U

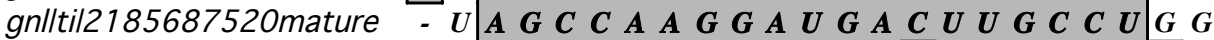
DR924236binding DR927955binding

- $A$ A

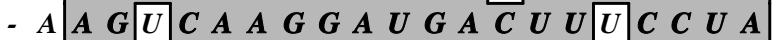

- $A$\begin{tabular}{lllllllllllllllllllll}
$A$ & $G$ & $U$ & $C$ & $A$ & $A$ & $G$ & $G$ & $A$ & $U$ & $G$ & $A$ & $C$ & $U$ & $U$ & $U$ & $C$ & $C$ & $U$ & $A$ \\
\hline
\end{tabular}

G W A G C C A A G G A U G A C UU G C C U A

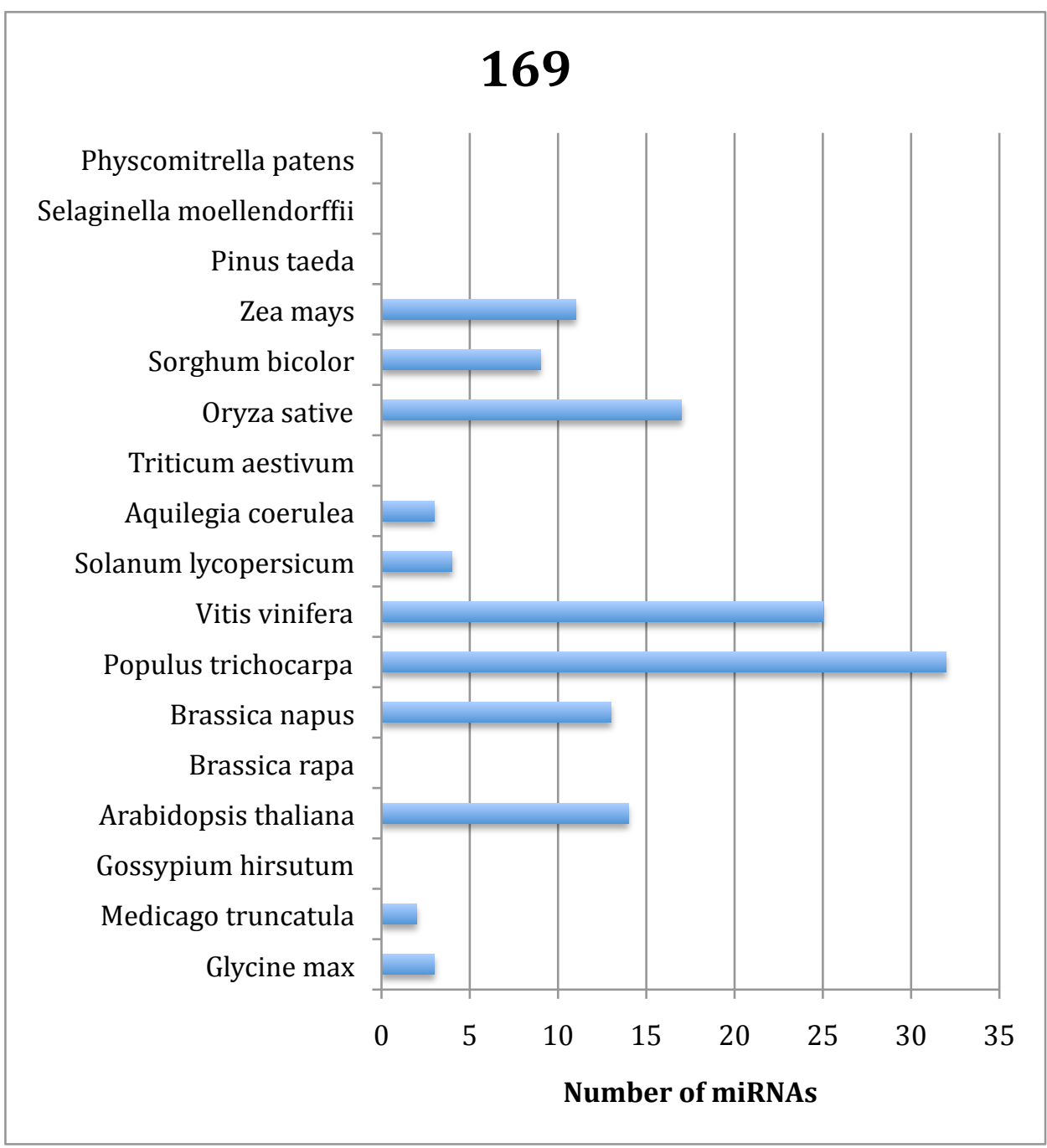


microRNA169

Output of sir_graph (๑)

Created Thu Apr 206:50:07 2009

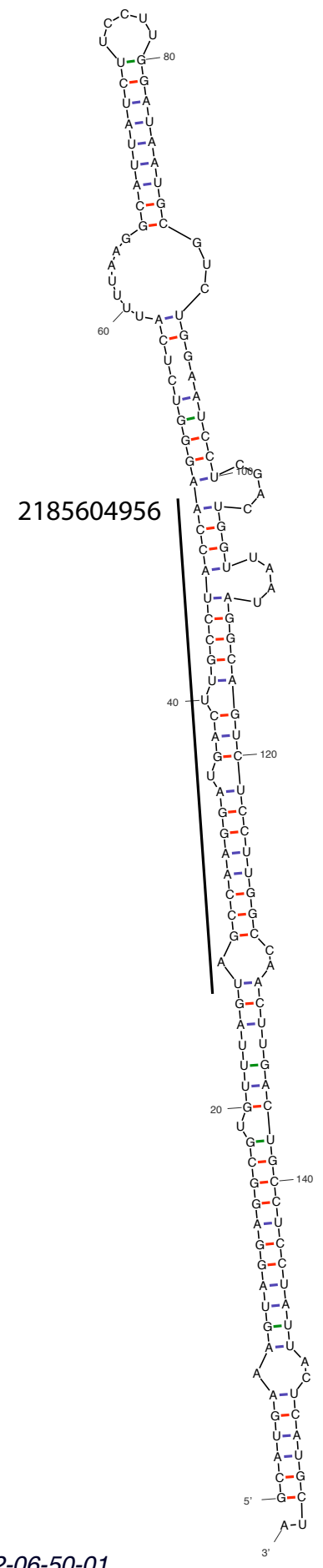

$d G=-75.30$ [initially -75.30$]$ 09Apro2-06-50-01

microRNA169 
microRNA169

Output of sir_graph (๑)

Created Thu Apr 207:06:06 2009

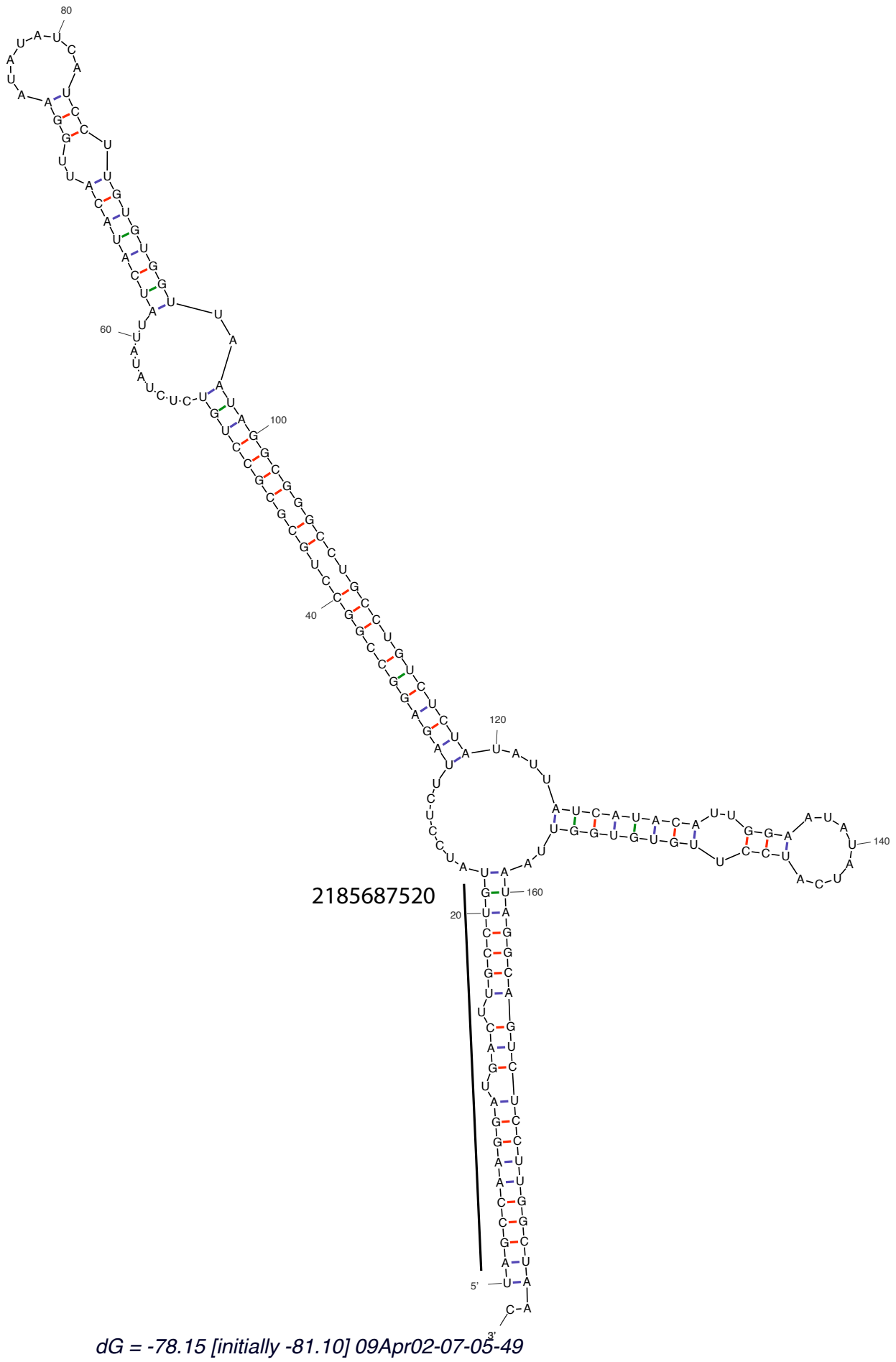

microRNA169 
microRNA169

Output of sir_graph (®)

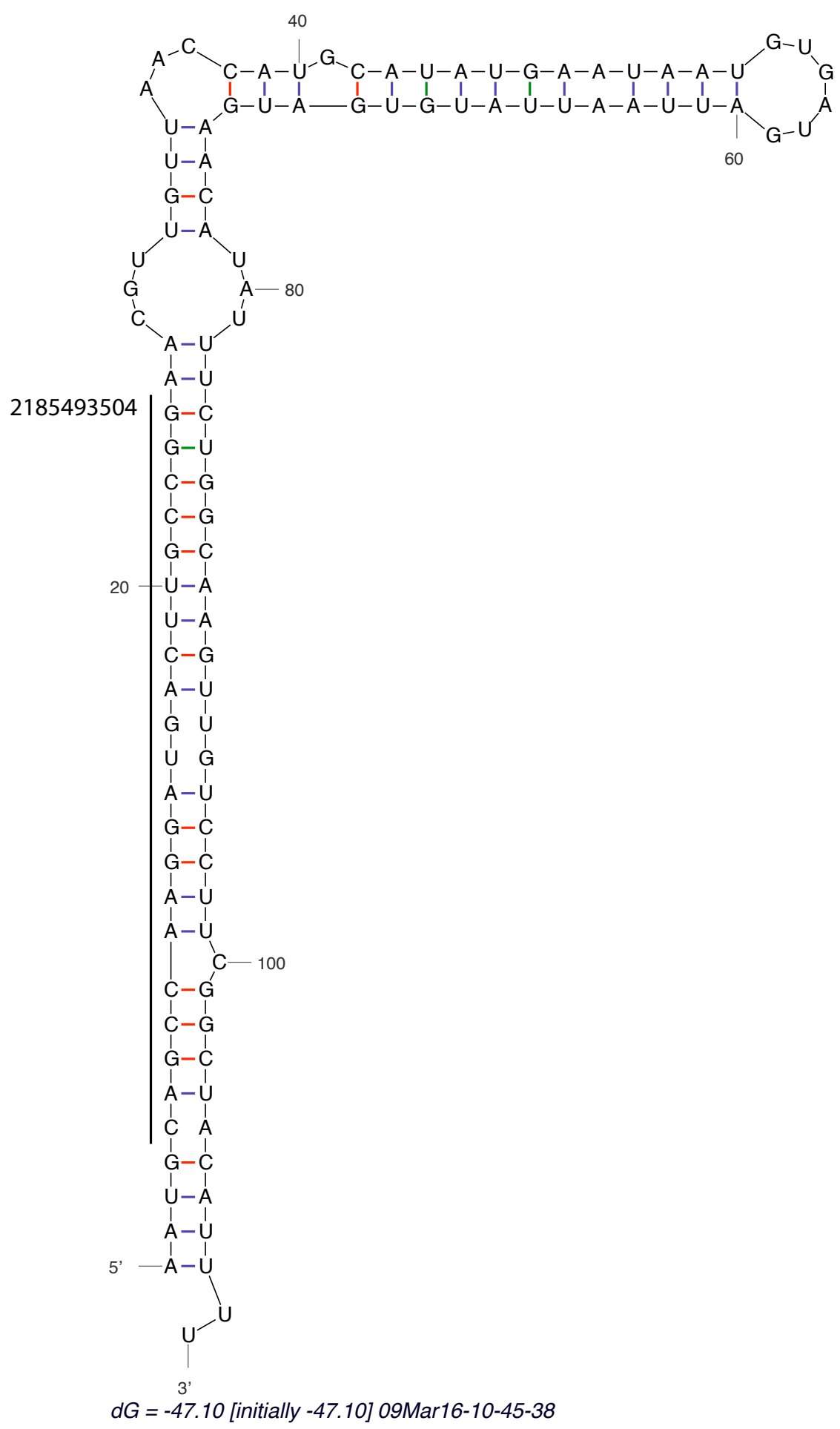

microRNA169 
microRNA170/171

20

2185468845mature
2185568457 mature
2185624710 mature
2185689689 mature
2185735537 mature
2185815653 mature
TC21487binding
TC25185binding
TC28359binding
TC29397binding

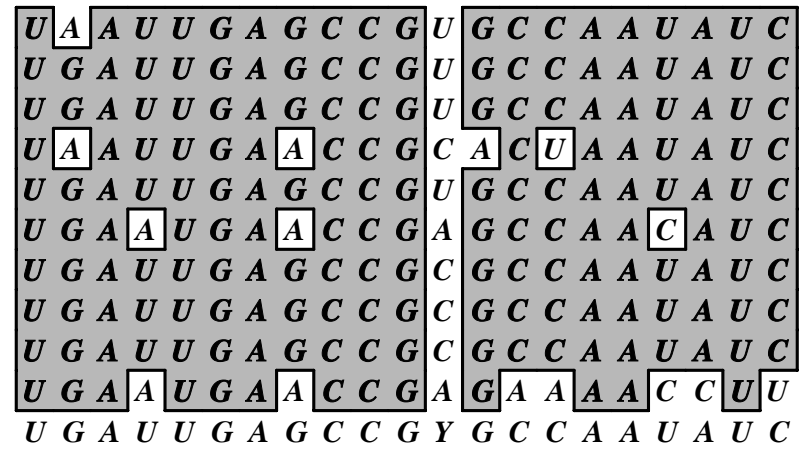

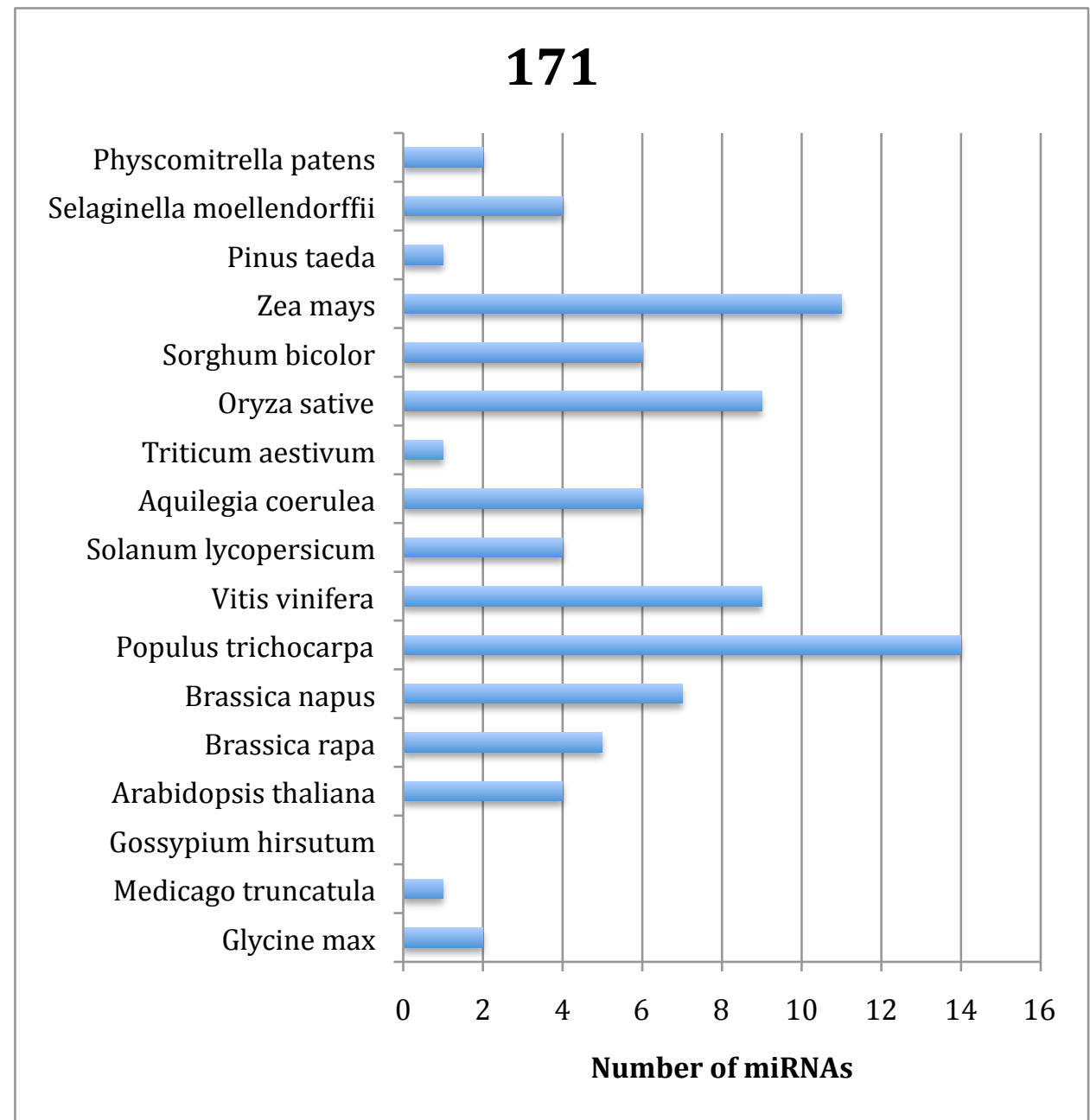

microRNA170/171 
microRNA170/171

Output of sir_graph ()

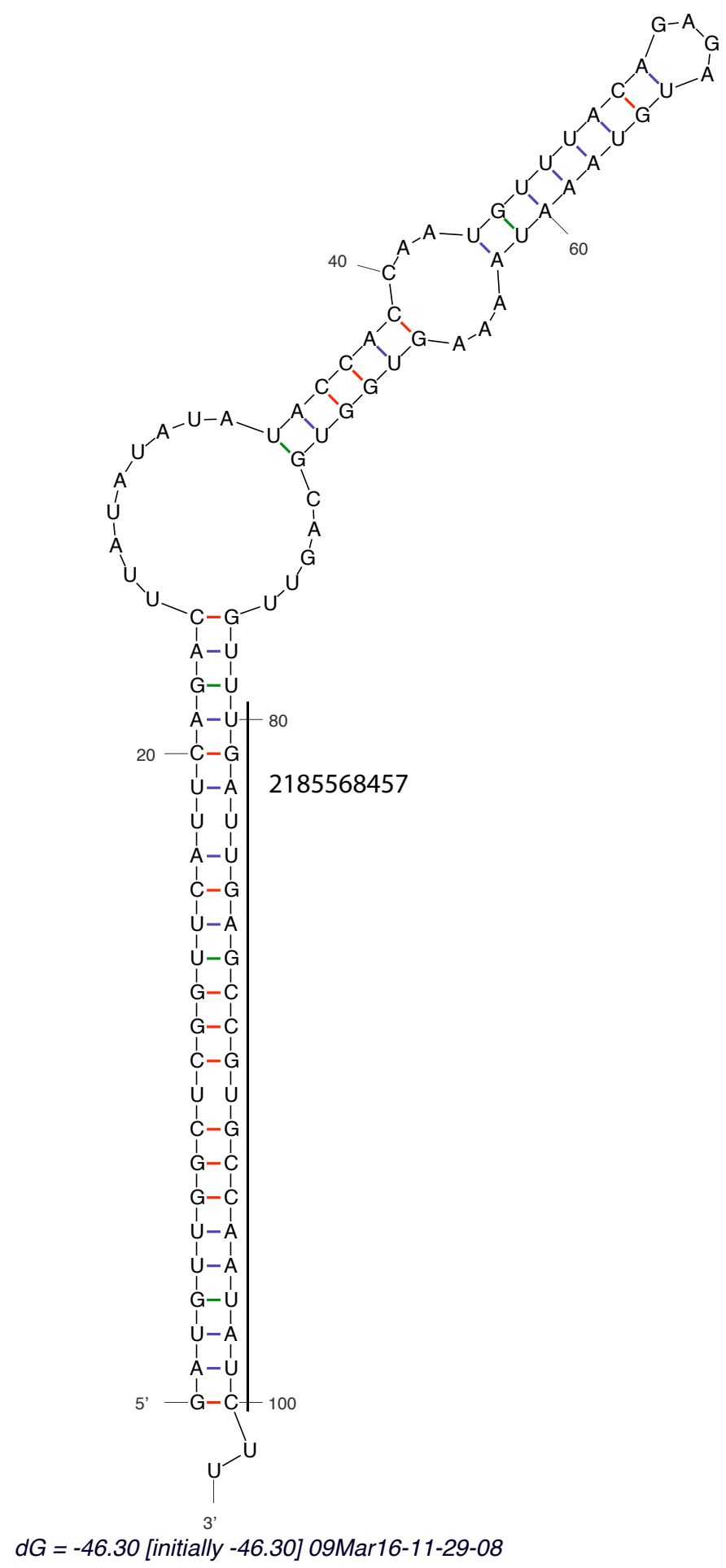

microRNA170/171 
microRNA170/171

Output of sir_graph (@)
mfold 3.4

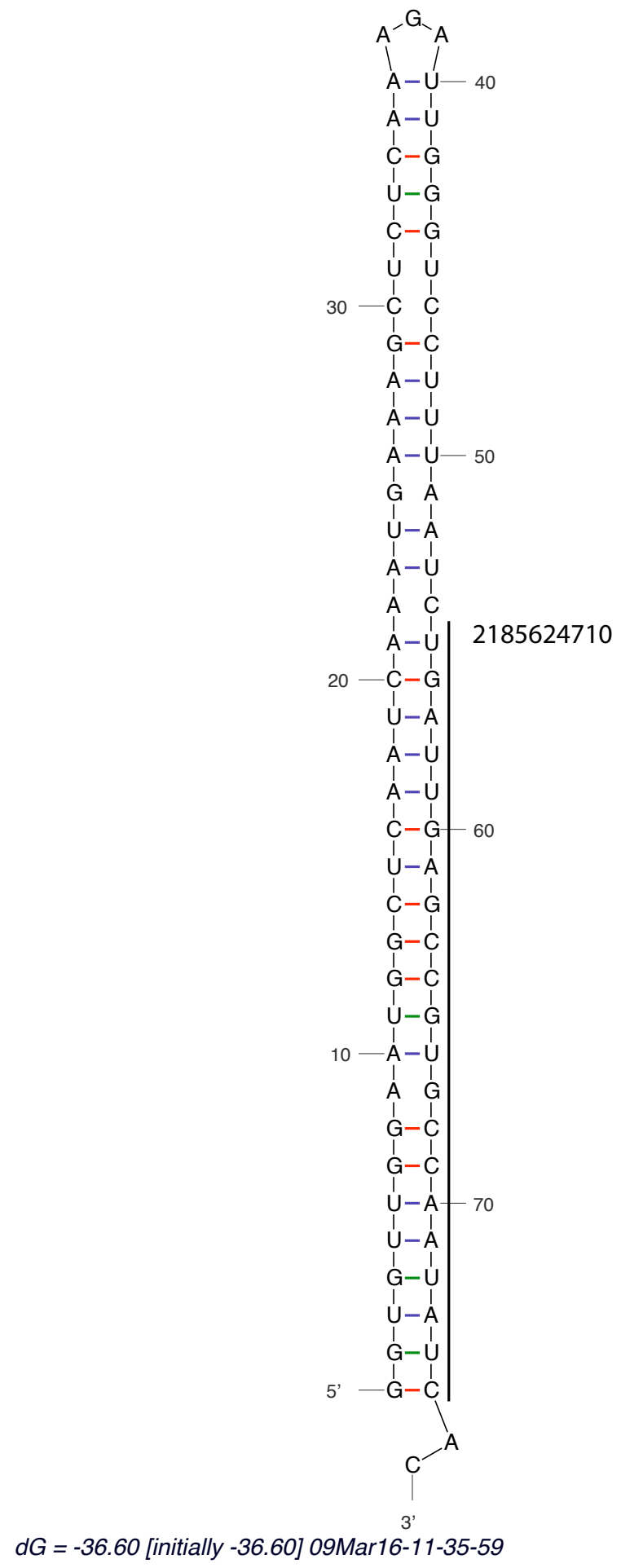

microRNA170/171 
microRNA170/171

Output of sir_graph (®)

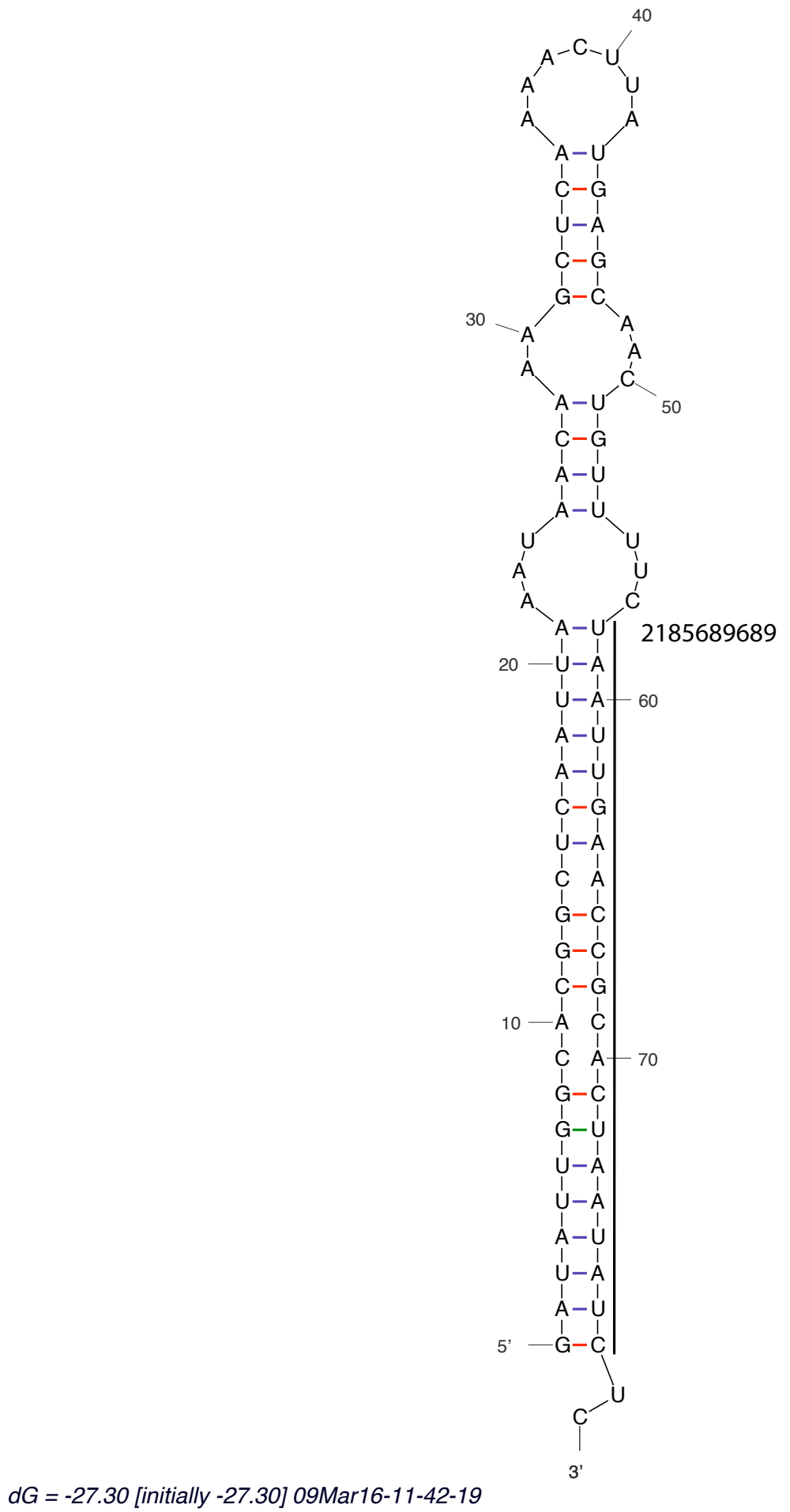

microRNA170/171 
microRNA170/171

Output of sir_graph (®)

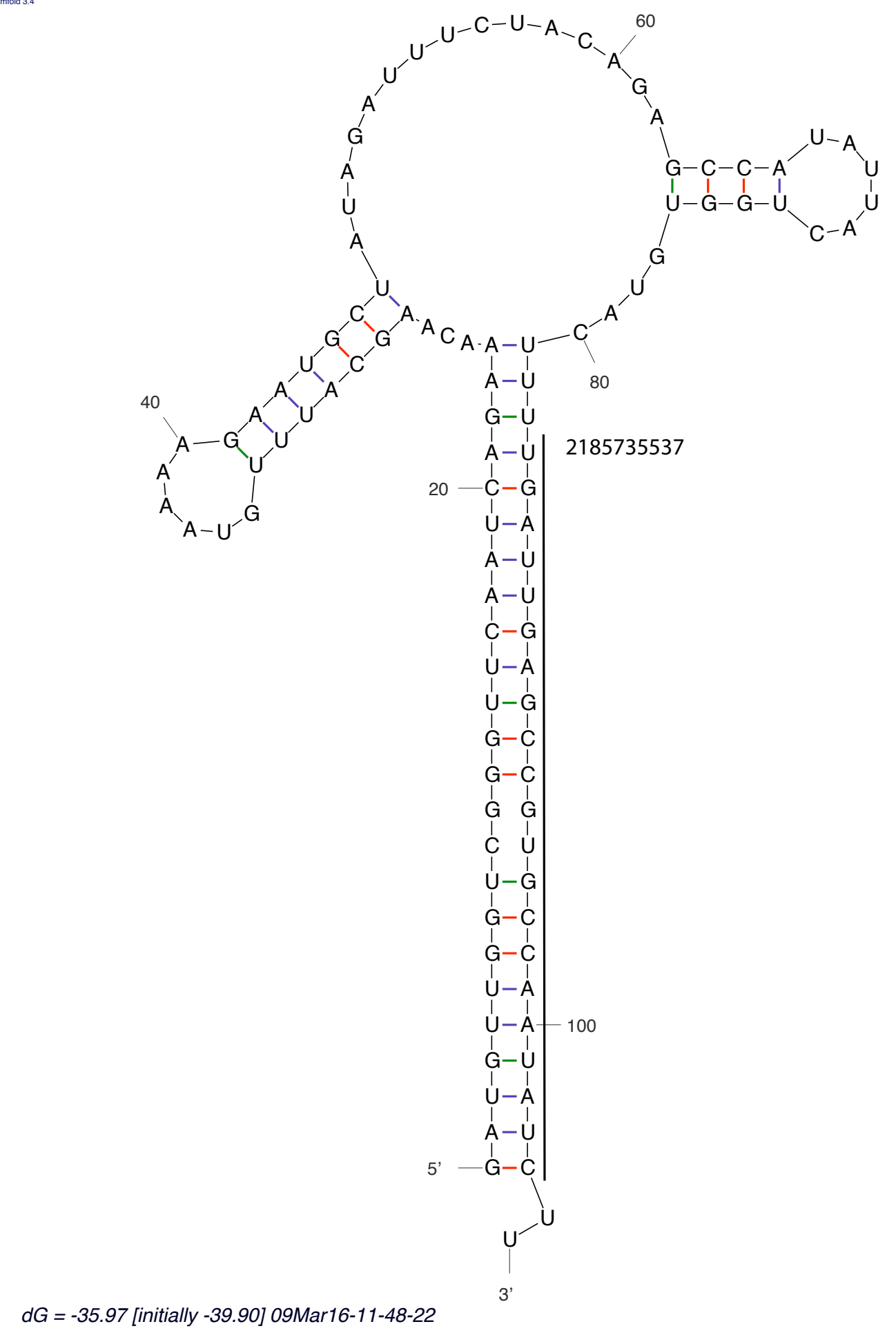

microRNA170/171 
microRNA170/171

Output of sir_graph (ㅇ)

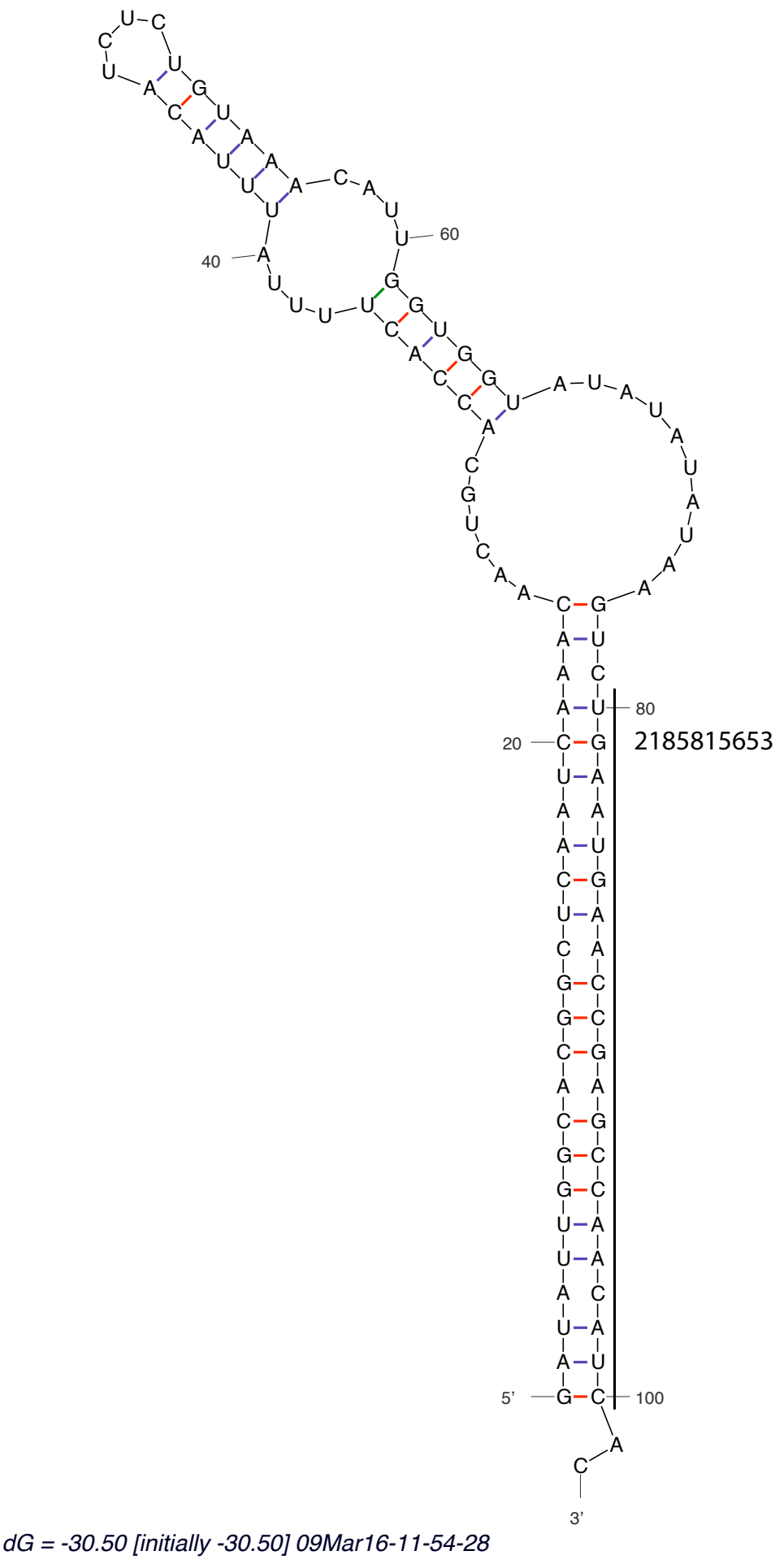

microRNA170/171 
microRNA170/171

Output of sir_graph (ㅇ)

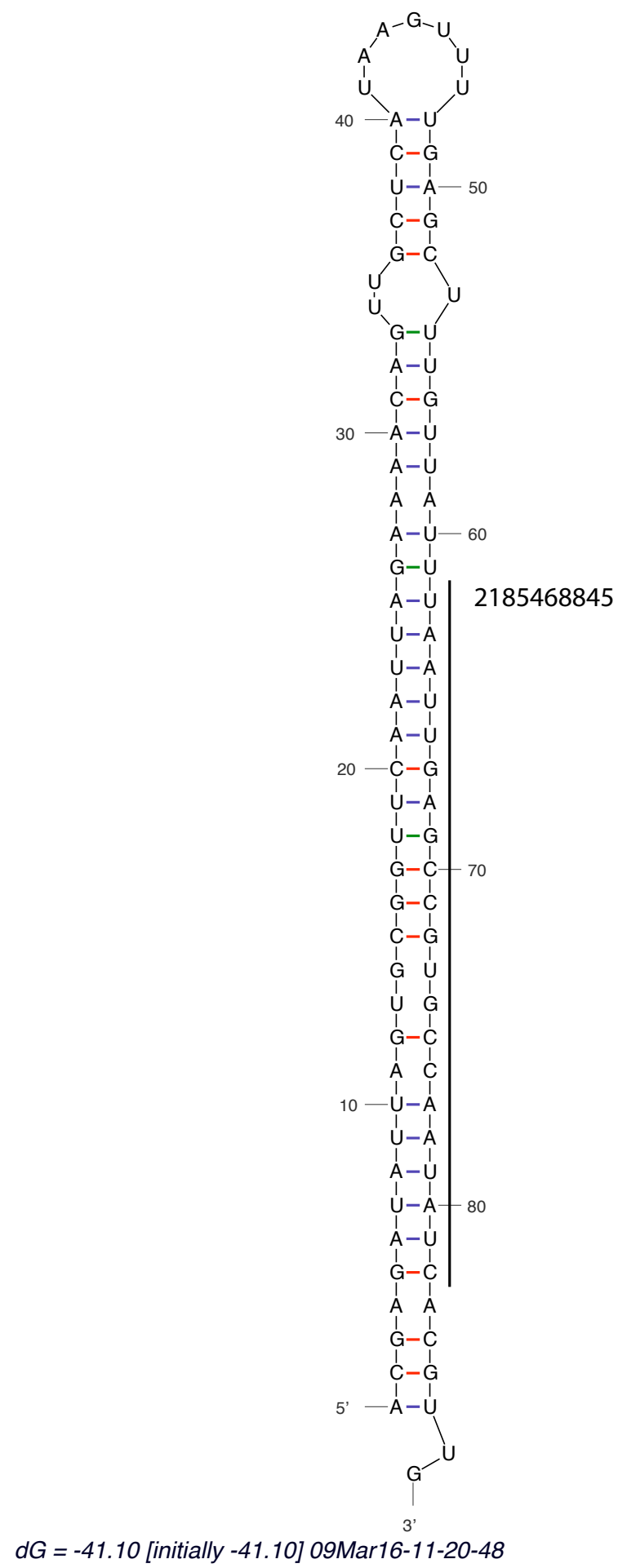

microRNA170/171 
microRNA172

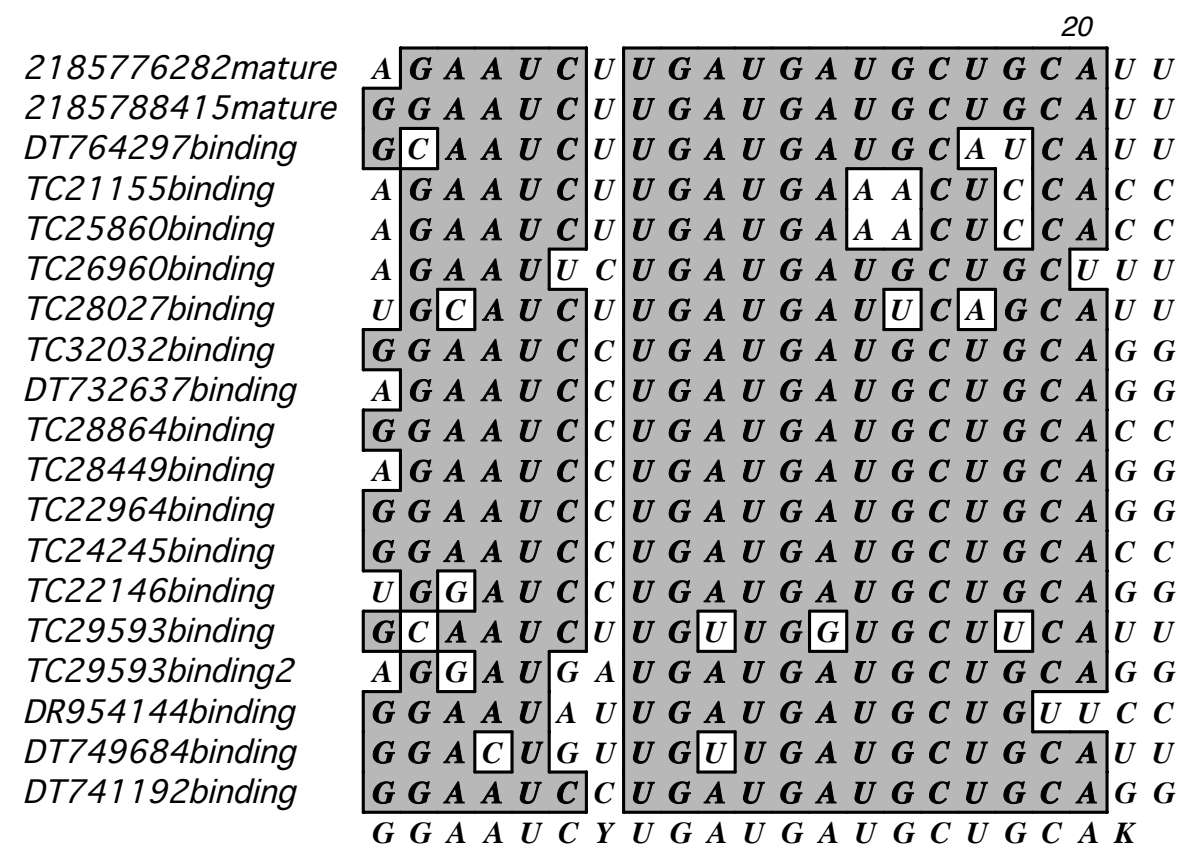

microRNA172 
microRNA172

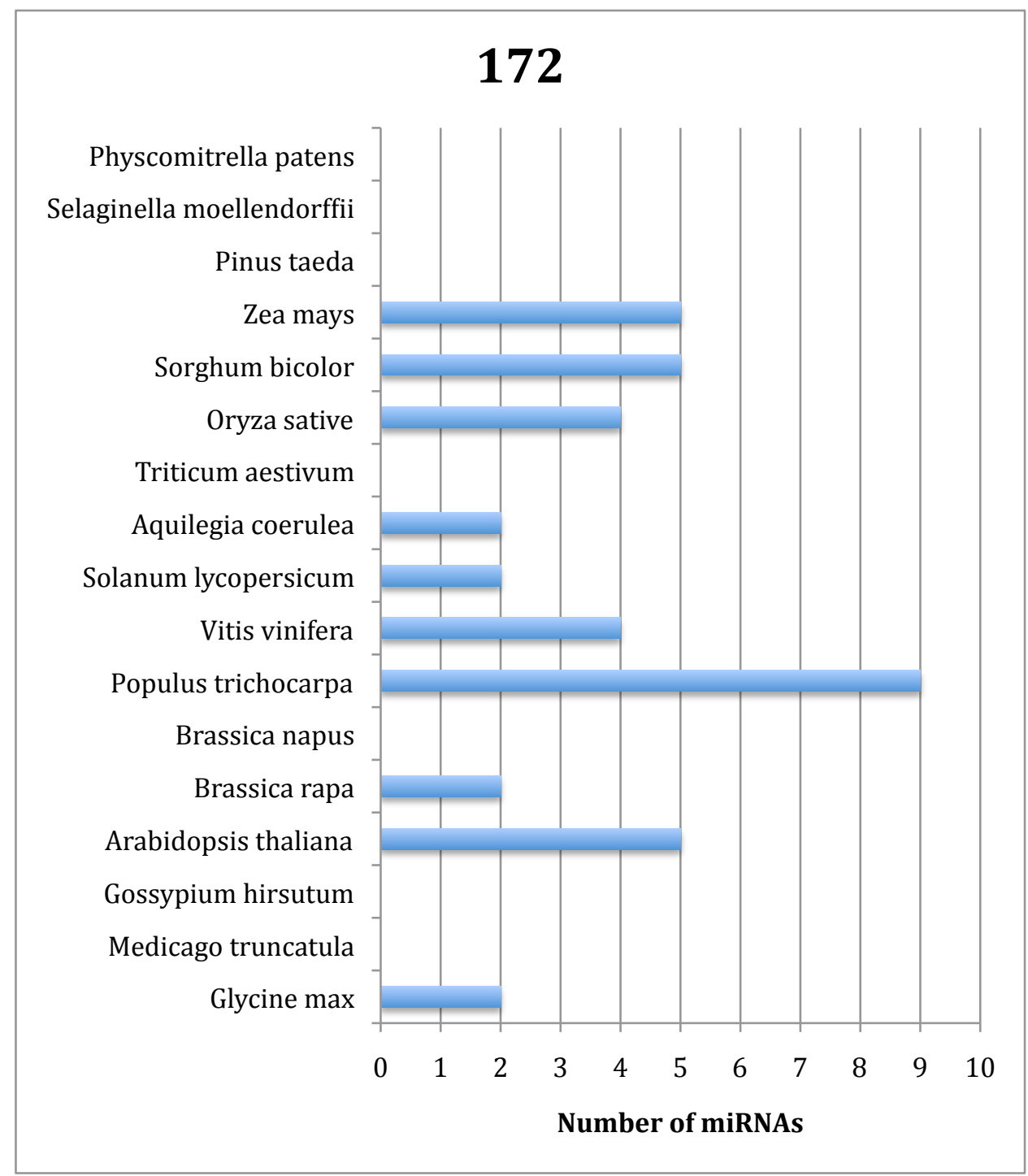


microRNA172

Output of sir_graph (๑)

Created Tue May 26 09:40:16 2009

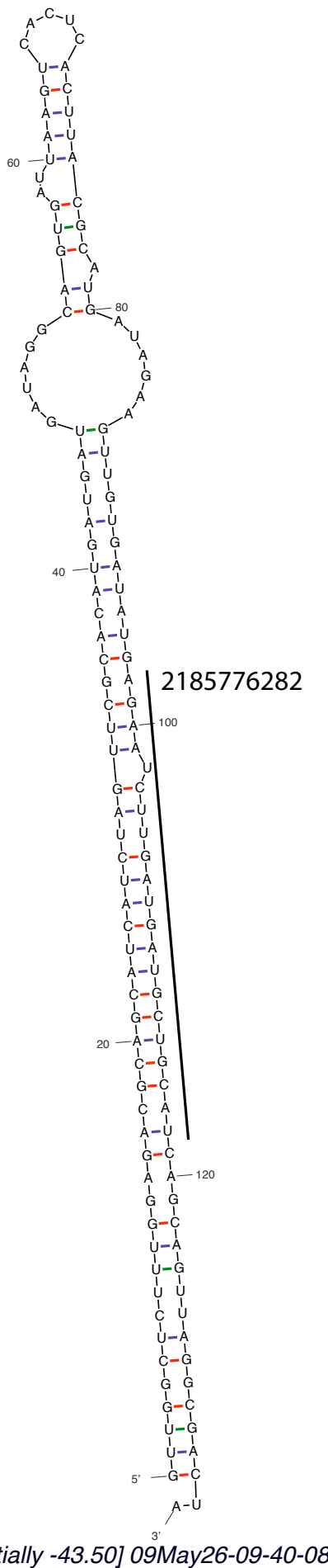

microRNA172 
microRNA172

Output of sir_graph (ㅇ)

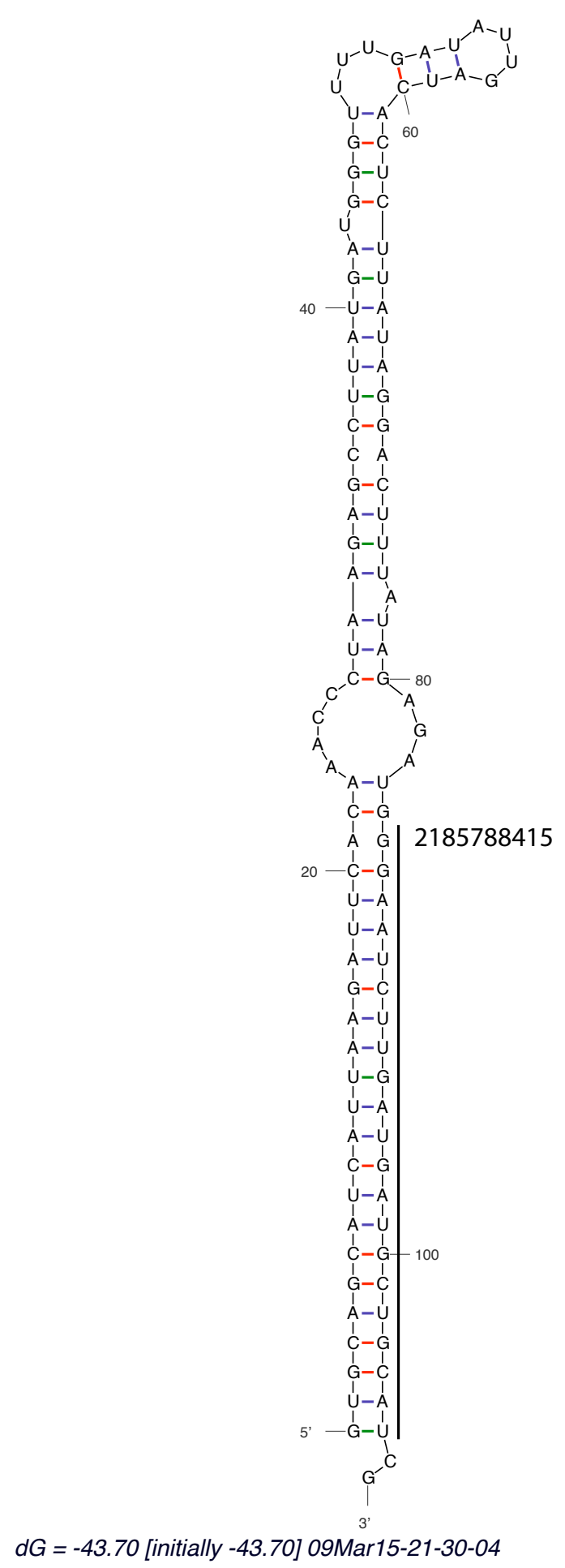

microRNA172 
microRNA319

\begin{tabular}{|c|c|}
\hline ינסיק & 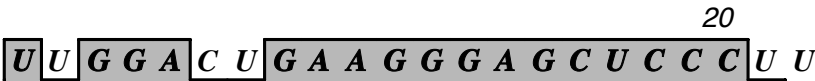 \\
\hline $922754 b$ & $U G G G A U A G A A B G G$ \\
\hline & $\begin{array}{lllllllllllllllllllll}U & G & G & G & A & U & A & G & A & A & G & G & G & A & G & C & U & C & C & C & A\end{array}$ \\
\hline
\end{tabular}

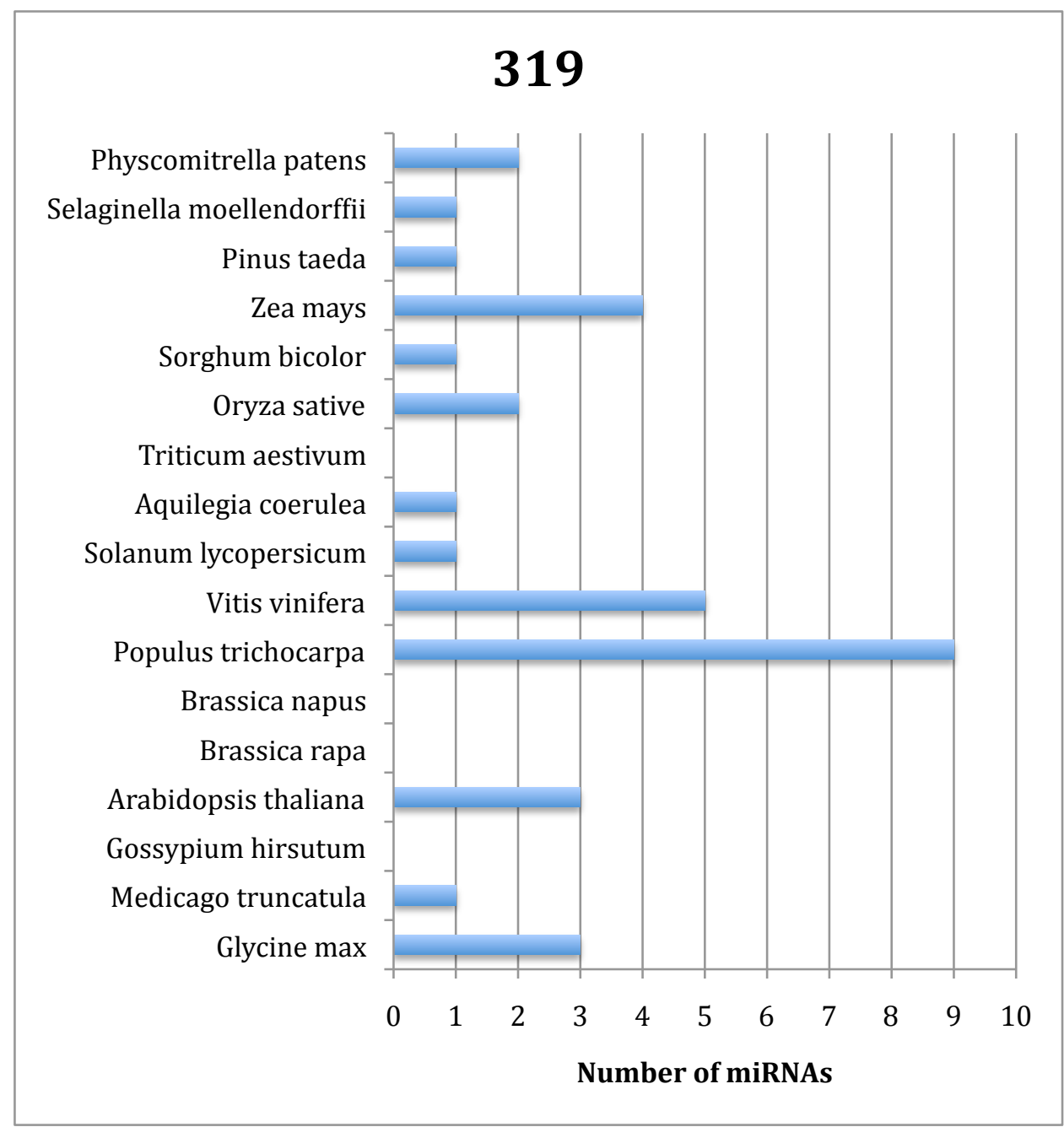


microRNA319

Output of sir_graph (®)
mfold 3.4

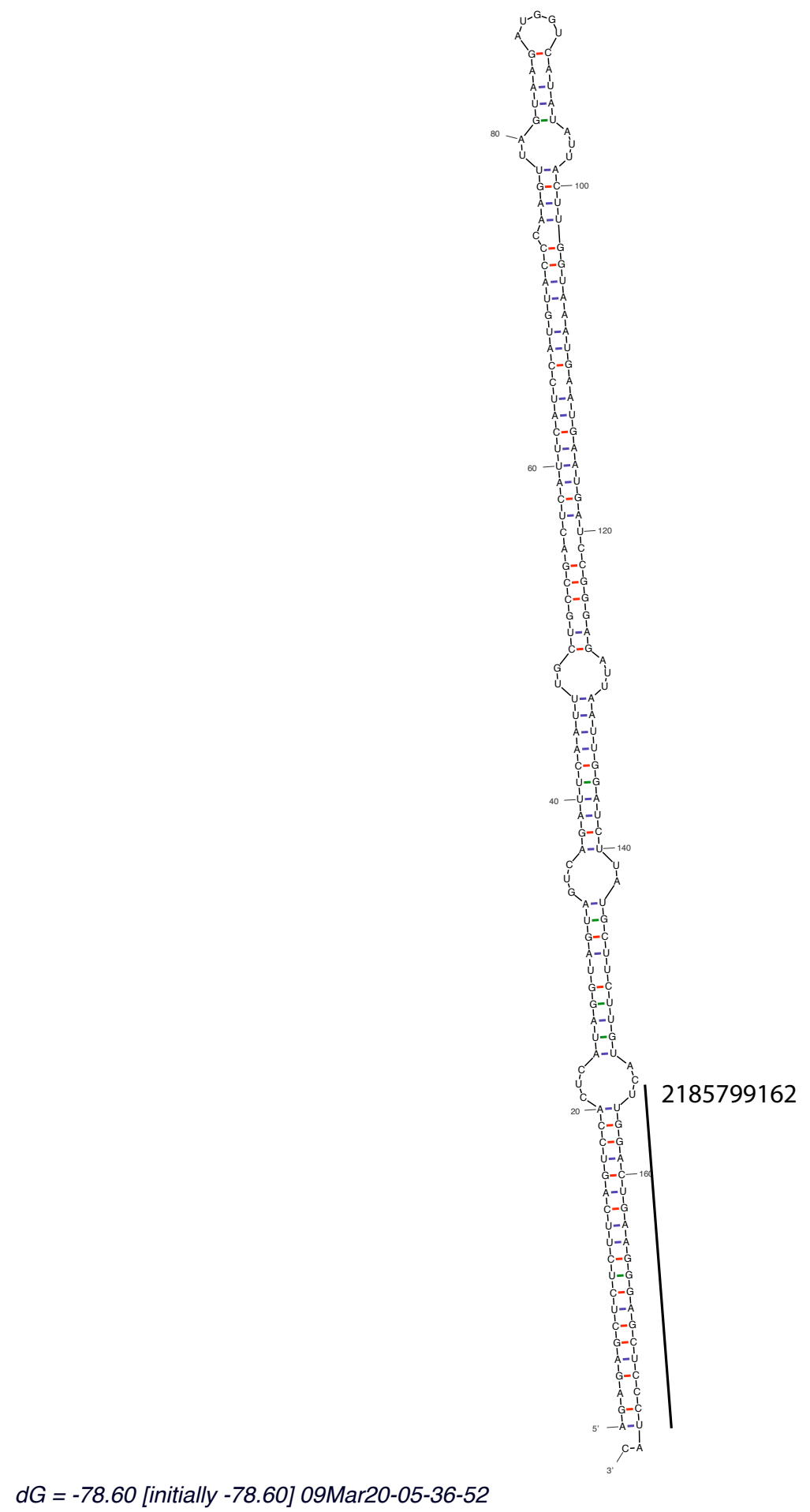

microRNA319 
microRNA390

20

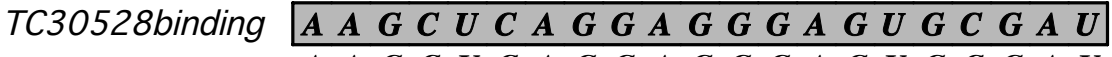

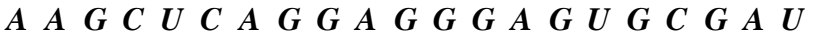

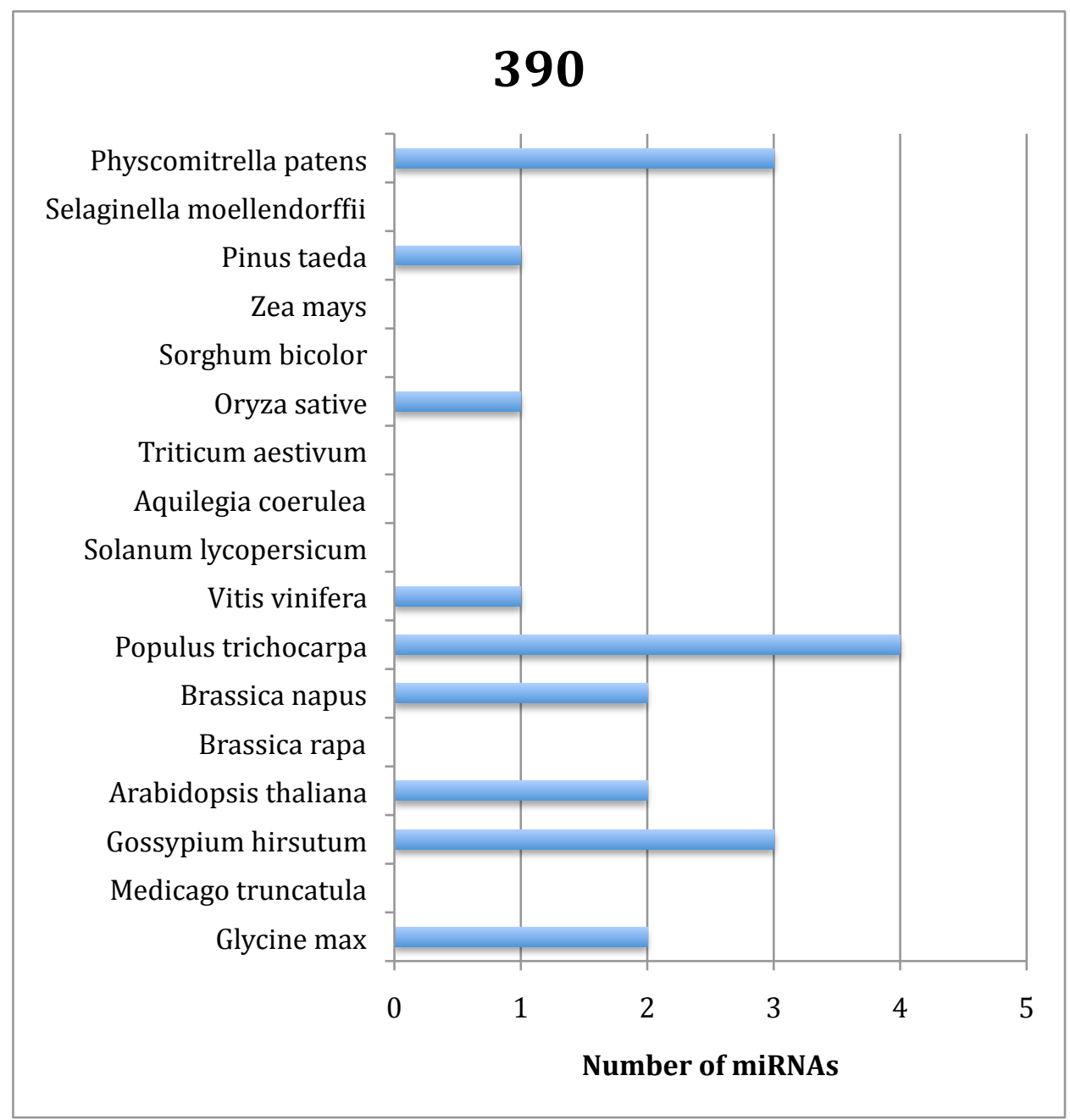


microRNA393

20

TC20718binding

TC25434binding

DR913737binding

TC29517binding

\begin{tabular}{lllllllllllllllllllllll}
\hline & $C$ & $C$ & $C$ & $A$ & $A$ & $A$ & $G$ & $G$ & $G$ & $A$ & $U$ & $C$ & $G$ & $C$ & $A$ & $U$ & $U$ & $G$ & $U$ & $C$ & $U$ & $C$
\end{tabular}

$\begin{array}{lllllllllllllllllllllll}\boldsymbol{U} & \boldsymbol{C} & \boldsymbol{C} & \boldsymbol{A} & \boldsymbol{A} & \boldsymbol{A} & \boldsymbol{G} & \boldsymbol{G} & \boldsymbol{G} & \boldsymbol{A} & \boldsymbol{U} & \boldsymbol{C} & \boldsymbol{G} & \boldsymbol{C} & \boldsymbol{A} & \boldsymbol{U} & \boldsymbol{U} & \boldsymbol{G} & \boldsymbol{U} & \boldsymbol{C} & \boldsymbol{U} & \boldsymbol{C}\end{array}$

$\begin{array}{llllllllllllllllllllll}U & C & C & A & A & A & G & G & G & A & U & C & G & C & A & U & U & G & U & C & U & C\end{array}$

\begin{tabular}{lllllllllllllllllllllll}
$\boldsymbol{U}$ & $\boldsymbol{C}$ & $\boldsymbol{C}$ & $\boldsymbol{A}$ & $\boldsymbol{A}$ & $\boldsymbol{A}$ & $\boldsymbol{G}$ & $\boldsymbol{G}$ & $\boldsymbol{G}$ & $\boldsymbol{A}$ & $\boldsymbol{U}$ & $\boldsymbol{C}$ & $\boldsymbol{G}$ & $\boldsymbol{C}$ & $\boldsymbol{A}$ & $\boldsymbol{U}$ & $\boldsymbol{U}$ & $\boldsymbol{G}$ & $\boldsymbol{U}$ & $\boldsymbol{U}$ & $\boldsymbol{U}$ & $\boldsymbol{C}$ \\
\hline
\end{tabular}

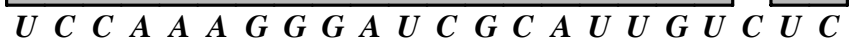

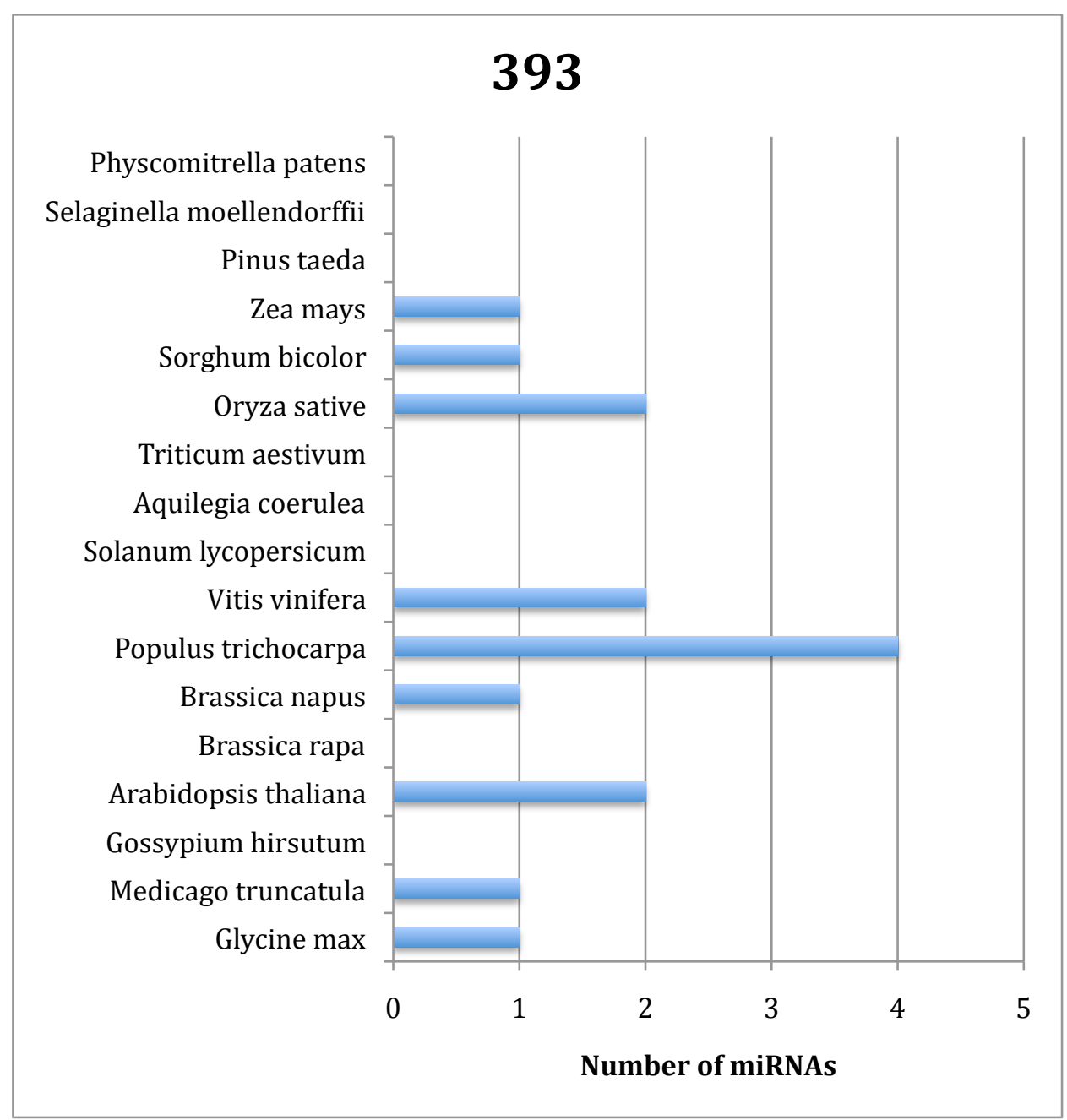


microRNA394

20

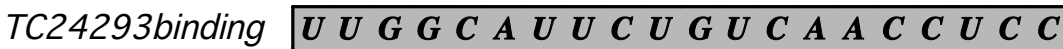

TC26097binding $U \begin{array}{llllllllllllllllllll}\boldsymbol{U} & \boldsymbol{U} & \boldsymbol{G} & \boldsymbol{G} & \boldsymbol{C} & \boldsymbol{A} & \boldsymbol{U} & \boldsymbol{U} & \boldsymbol{C} & \boldsymbol{U} & \boldsymbol{G} & \boldsymbol{U} & \boldsymbol{C} & \boldsymbol{A} & \boldsymbol{A} & \boldsymbol{C} & \boldsymbol{C} & \boldsymbol{U} & \boldsymbol{C} & \boldsymbol{C}\end{array}$

$\begin{array}{llllllllllllllllllll}U & U & G & G & C & A & U & U & C & U & G & U & C & A & A & C & C & U & C & C\end{array}$

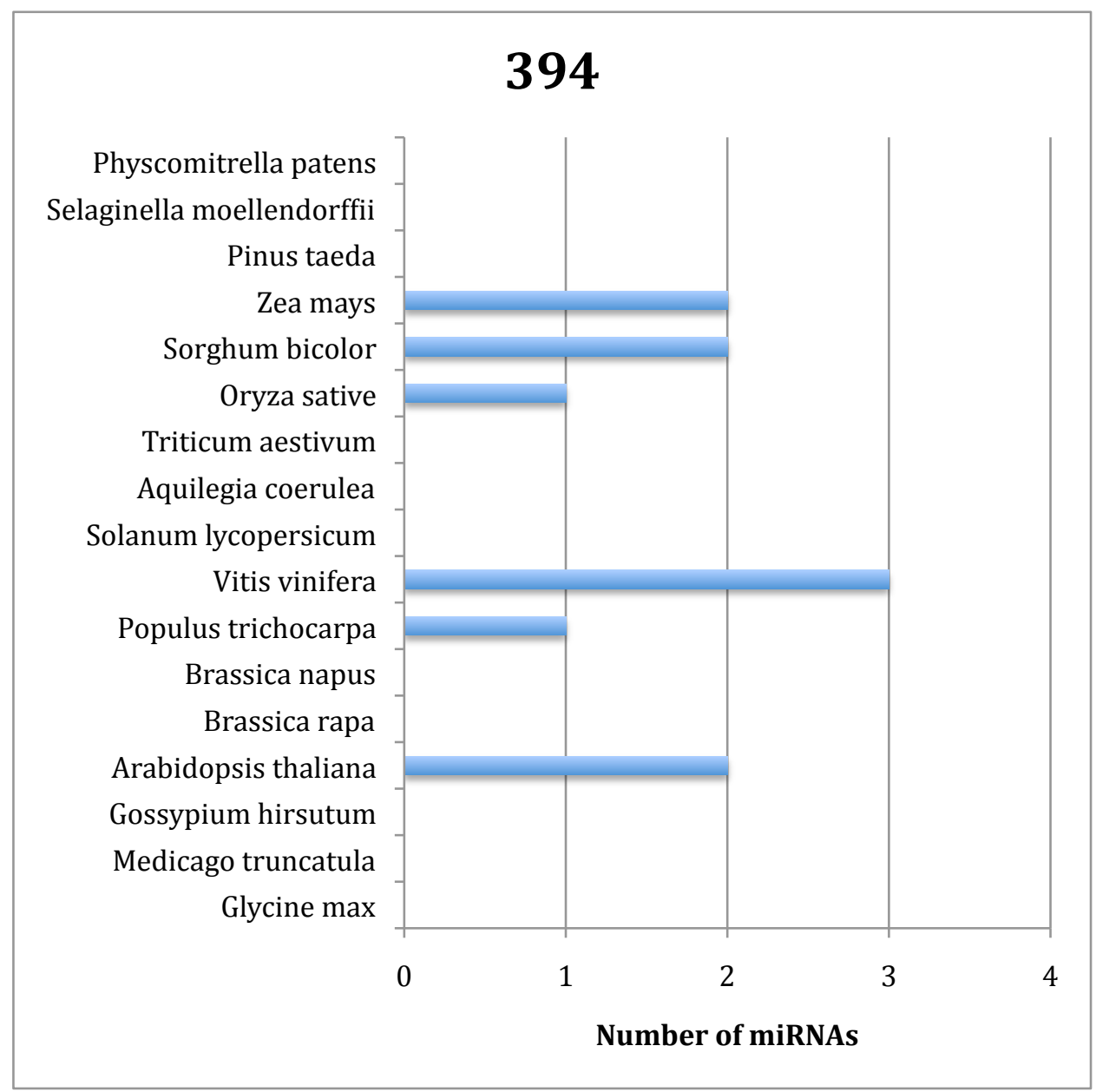


microRNA395

gnlltil2185894728mature gnlltil2185728750mature TC20934binding TC21492binding TC30052binding TC32489binding
$\begin{array}{llllllllllllllllllllll}C & U & G & A & A & G & G & G & U & U & U & G & G & A & G & G & A & A & C & U & C\end{array}$

$\begin{array}{lllllllllllllllllllll}C & U & G & A & A & G & G & G & U & U & U & G & G & A & G & G & A & A & C & U & C\end{array}$

$\begin{array}{lllllllllllllllllllllll}C & U & G & A & A & G & G & A & A & U & U & G & G & A & G & G & A & A & C & U & G & G\end{array}$ $A\left[\begin{array}{lllllllllllllllllllll}A & G & A & A & G & G & G & U & U & U & G & G & A & G & G & A & A & C & U & C\end{array}\right.$ $\begin{array}{llllllllllllllllllll}G & A & A & A & A & G & G & G & U & U & U & G & G & A & G & G & A & A & U & U\end{array}$ \begin{tabular}{|lllllllllllllllllllll}
\hline$C$ & $U$ & $G$ & $A$ & $A$ & $G$ & $G$ & $G$ & $U$ & $U$ & $U$ & $G$ & $G$ & $A$ & $G$ & $G$ & $A$ & $A$ & $C$ & $U$ & $C$
\end{tabular}

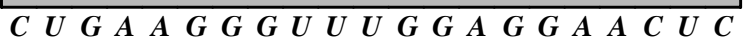

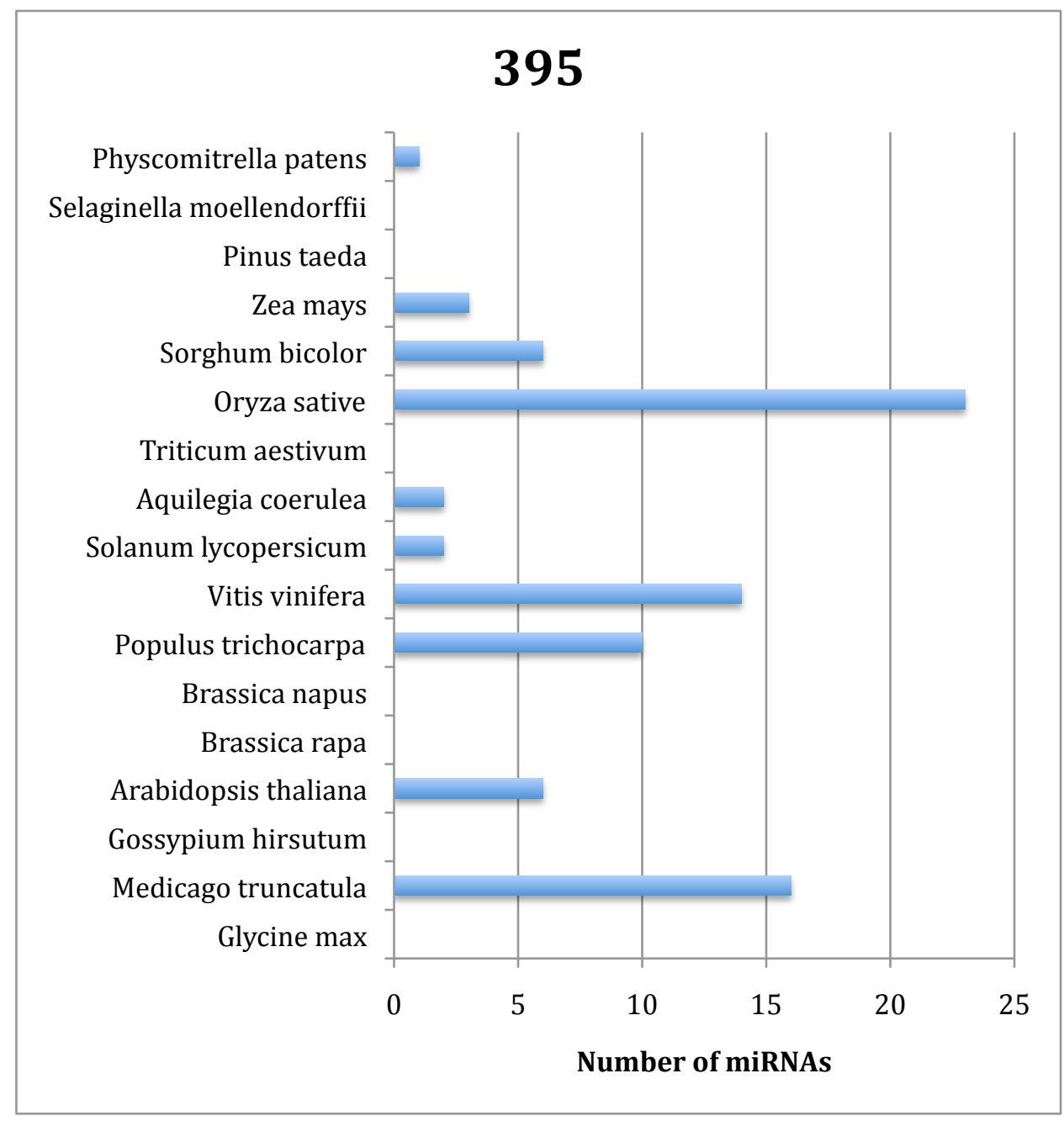


microRNA395

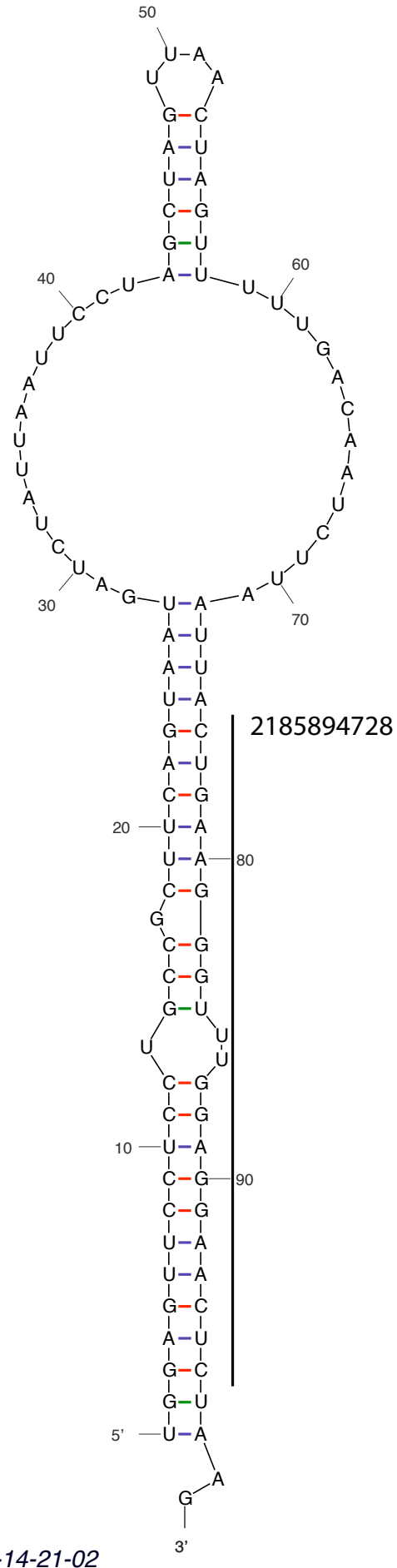

$d G=-43.60$ [initially -43.60 ] 09Apr06-14-21-02 
microRNA395

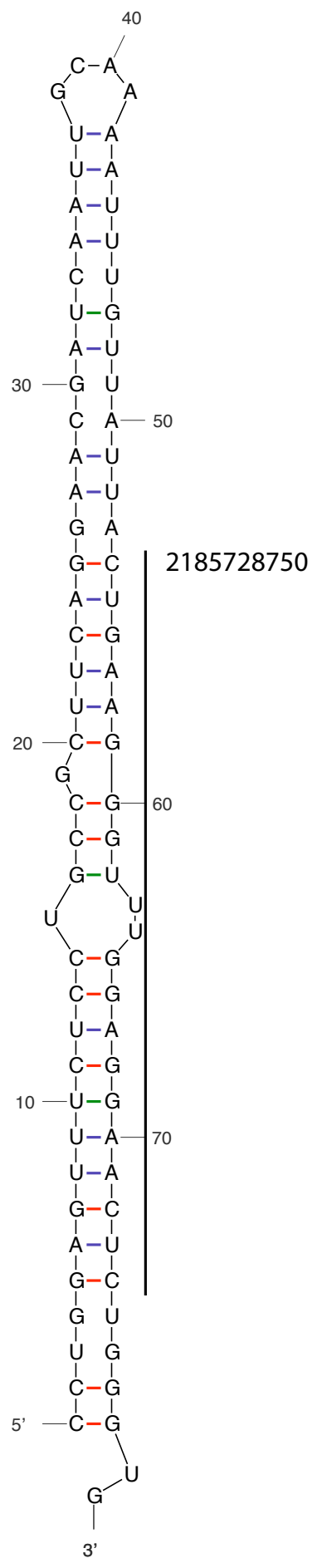


microRNA396

2185617799 mature
2185513426 mature
DR926810binding
TC20841 binding
TC22590binding
TC27254binding
TC29396binding
TC32094binding

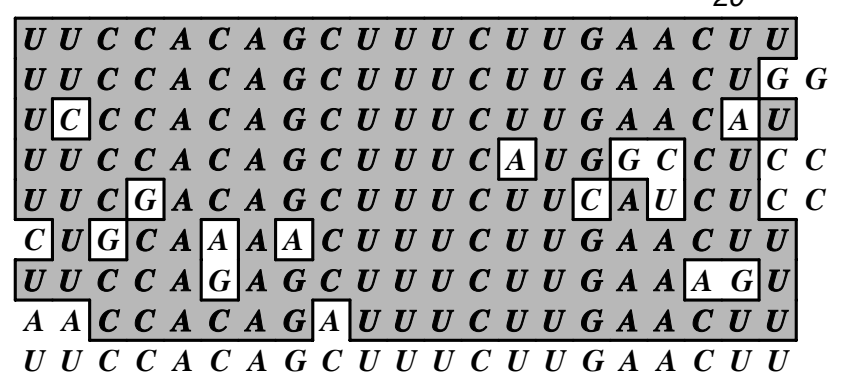

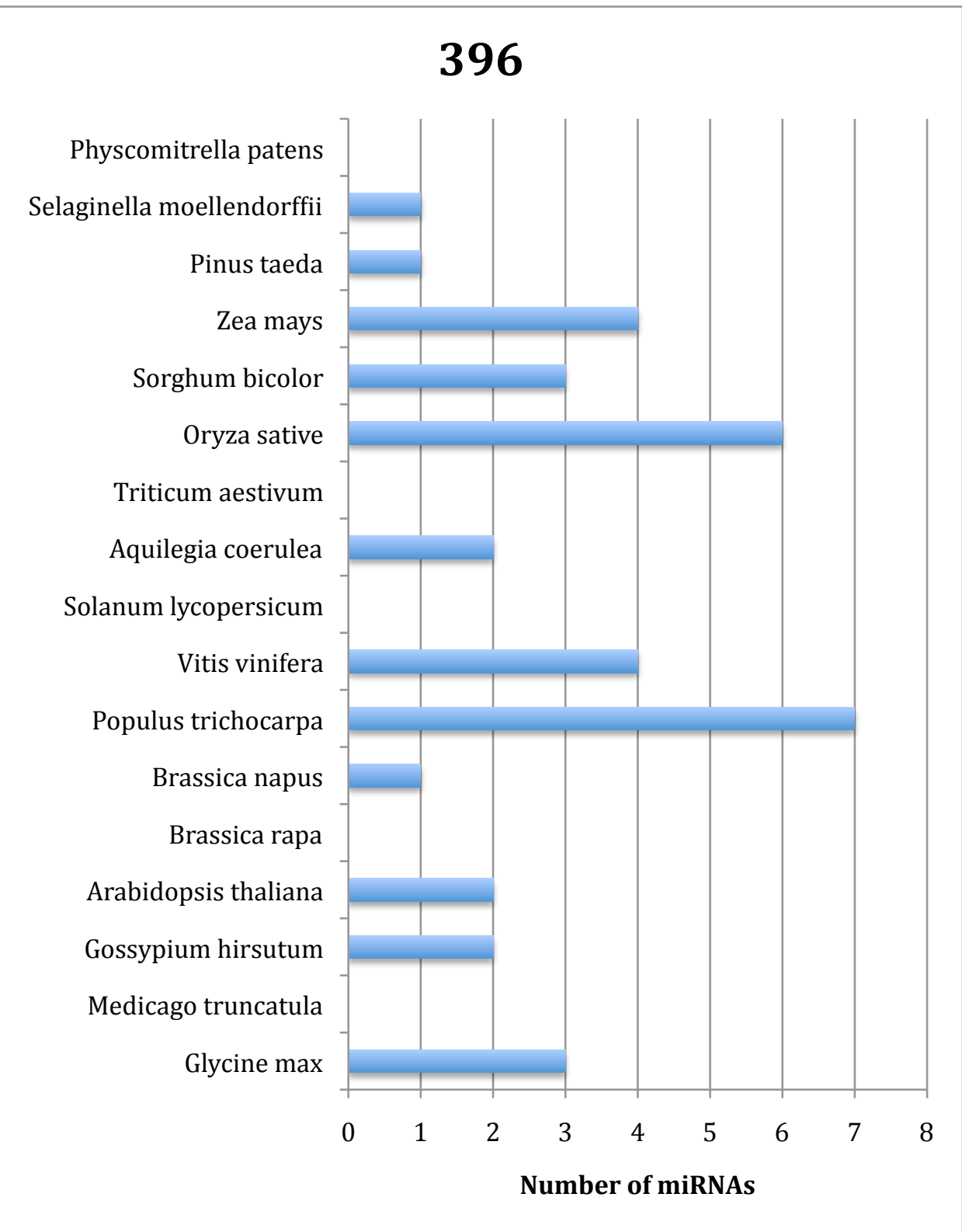


microRNA396

Output of sir_graph (ㅇ)

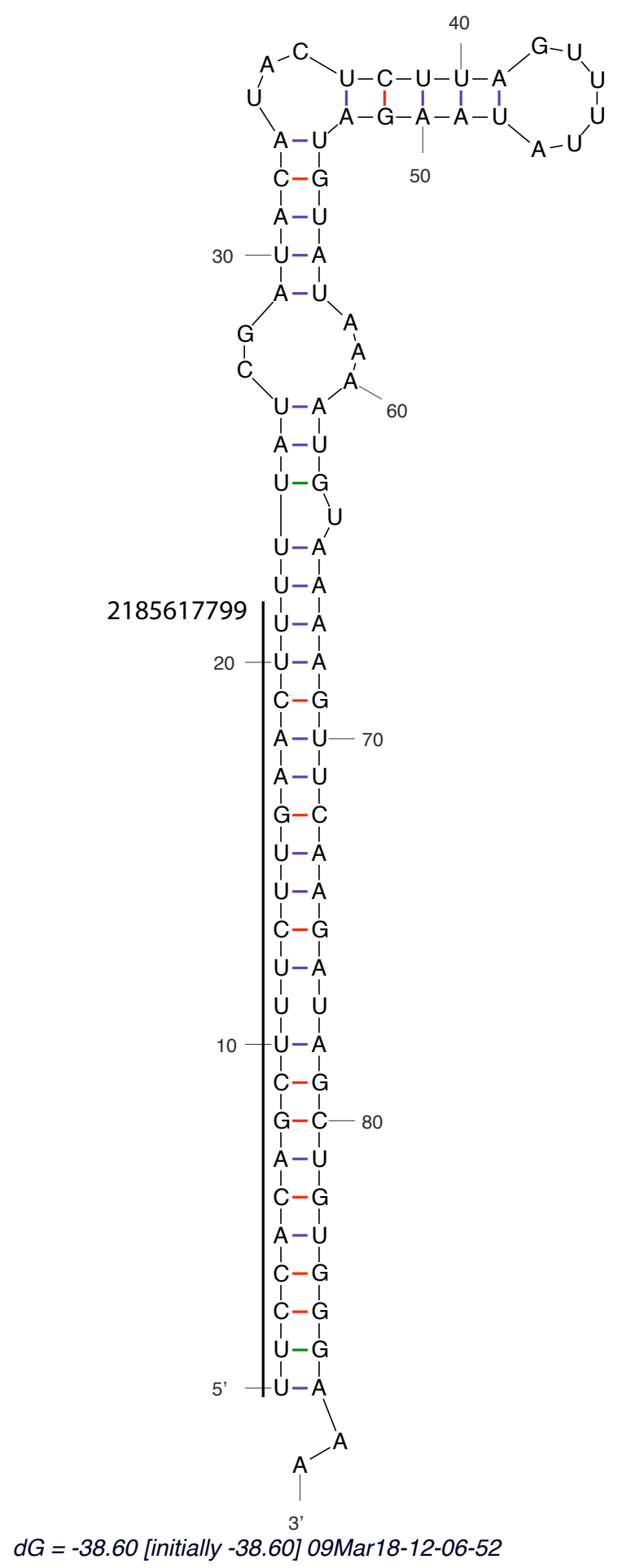

microRNA396 
microRNA396

Output of sir_graph (ㅇ)

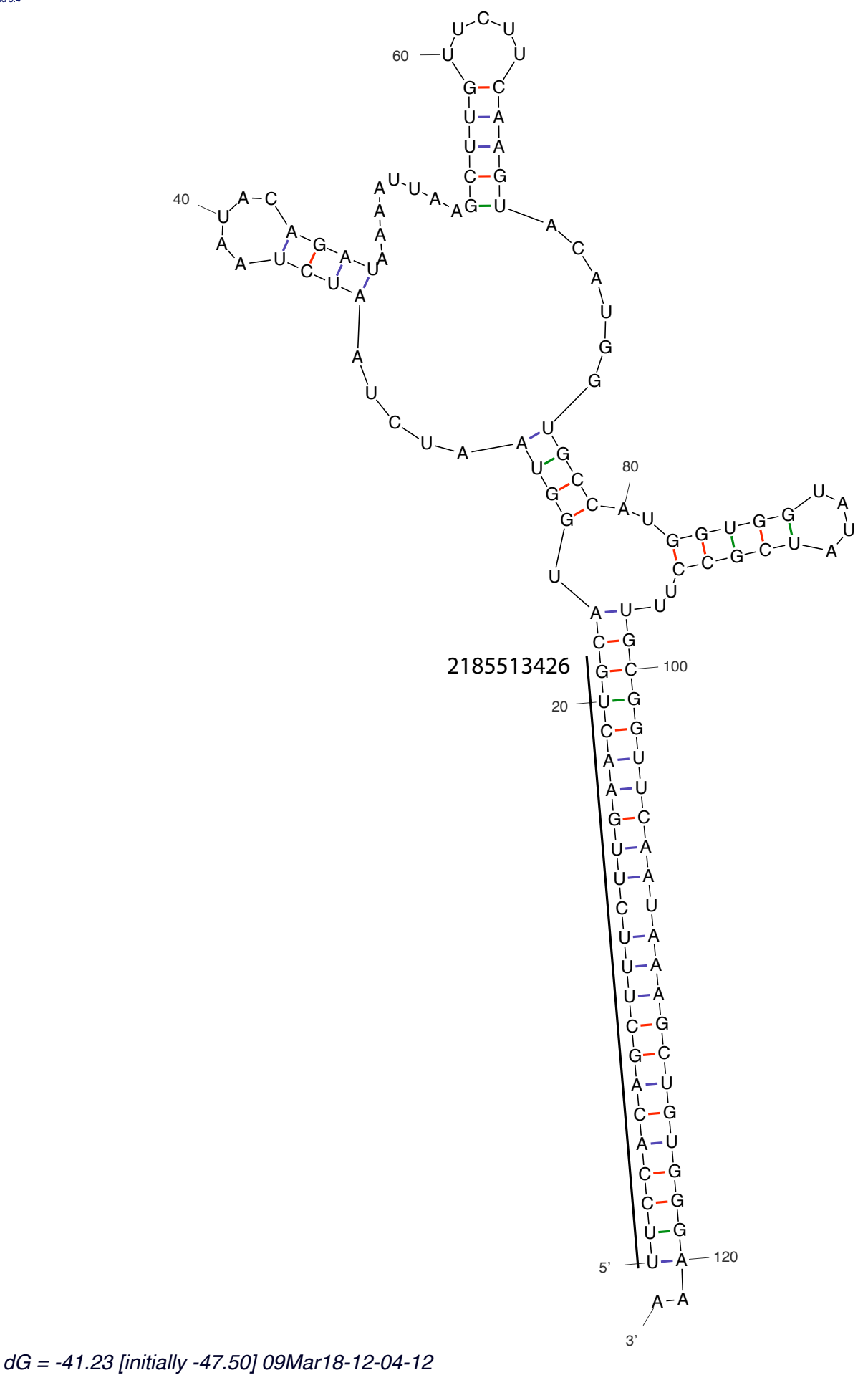

microRNA396 
microRNA397

TC26200binding

TC26926binding

DT728841binding

DT743386binding

TC24152binding

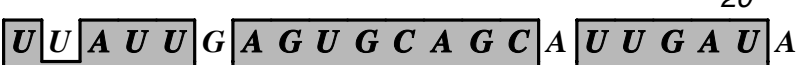

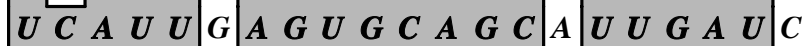

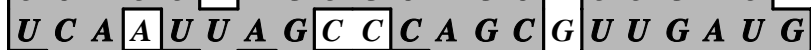

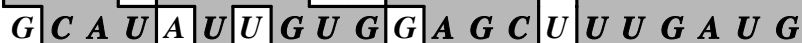

\begin{tabular}{lllll|l|l|lll|l|lll|l|llllll}
$G$ & $C$ & $A$ & $U$ & $A$ & $\boldsymbol{U}$ & $\boldsymbol{U}$ & $G$ & $\boldsymbol{U}$ & $\boldsymbol{G}$ & $\boldsymbol{G}$ & $\boldsymbol{A}$ & $\boldsymbol{G}$ & $\boldsymbol{C}$ & $\boldsymbol{U}$ & $\boldsymbol{U}$ & $\boldsymbol{U}$ & $\boldsymbol{G}$ & $\boldsymbol{A}$ & $\boldsymbol{U}$ & $\boldsymbol{G}$
\end{tabular}

UCAUUUA GUG CA GCWUUGA

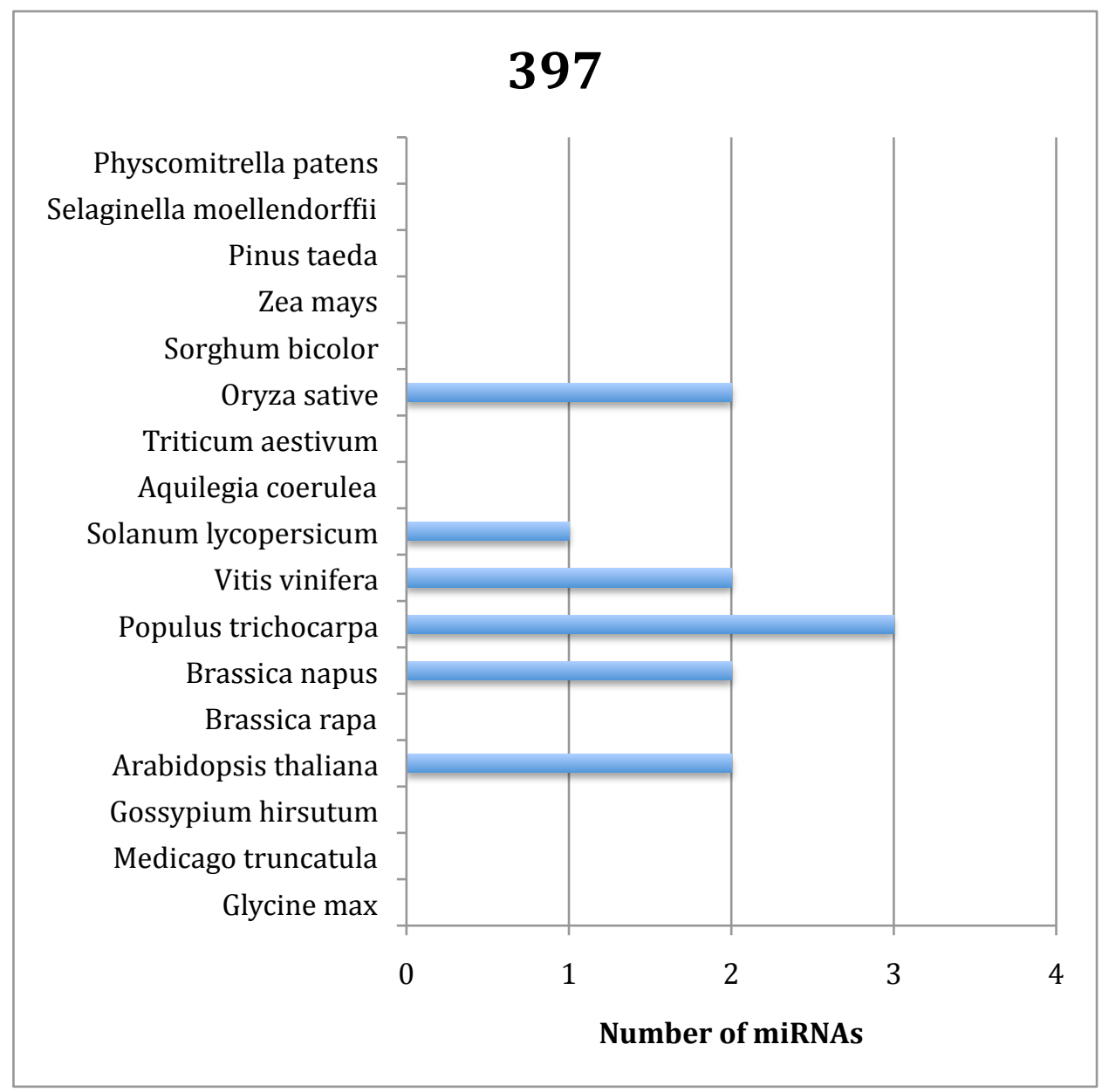


microRNA398
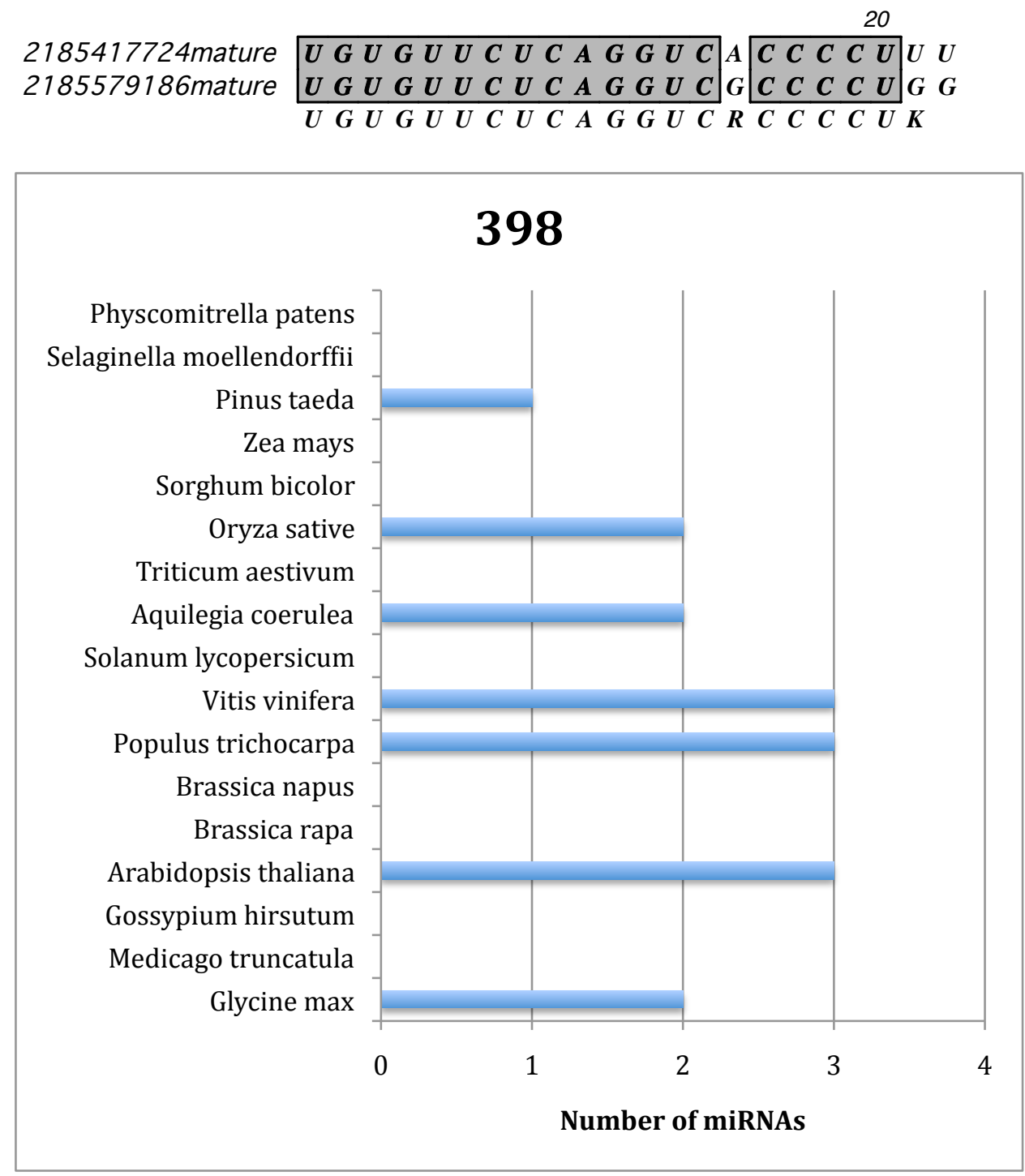
microRNA398

Output of sir_graph (@)
mfold 3.4

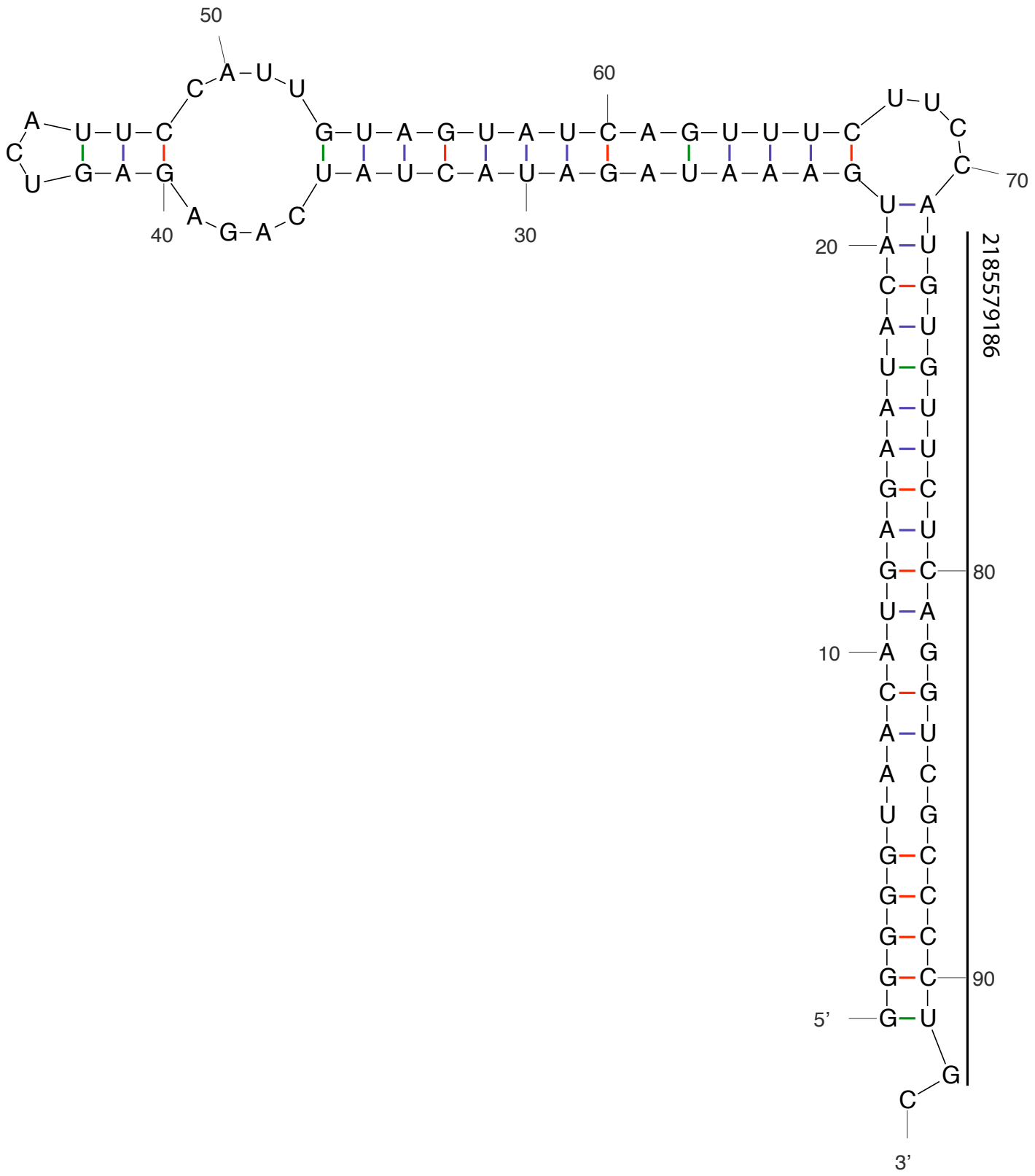

$d G=-38.50$ [initially -38.50$]$ 09Mar18-21-55-00 
microRNA398

Output of sir_graph (@)
mfold 3.4

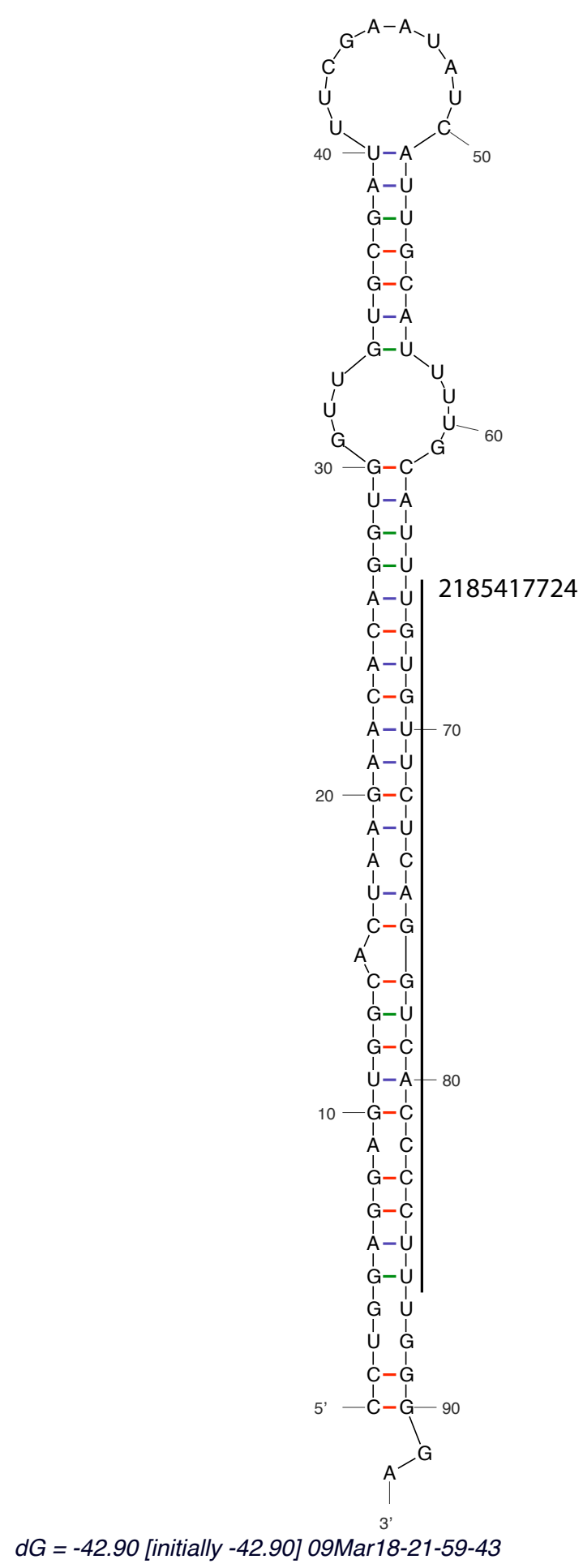

microRNA398 
microRNA399
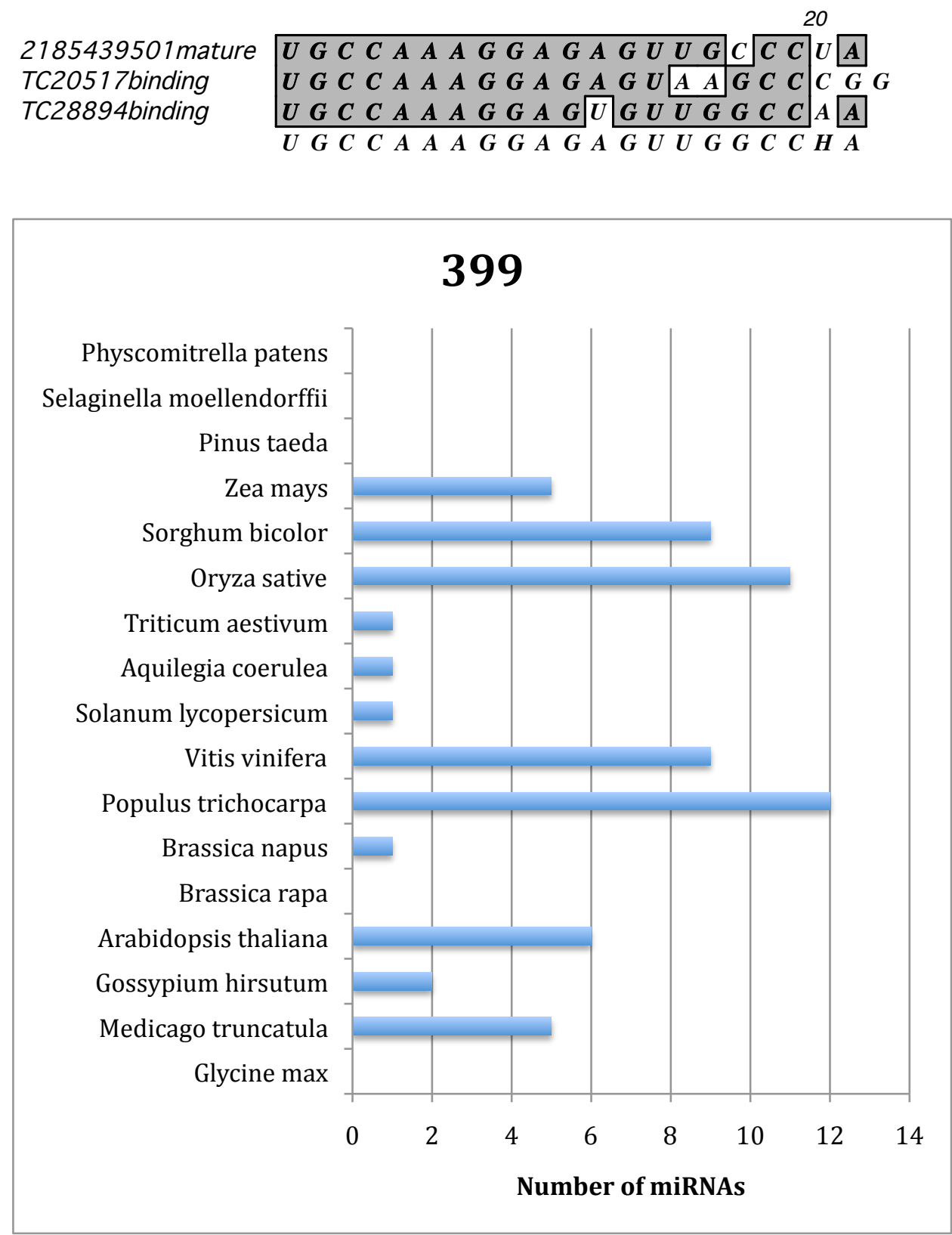
microRNA399

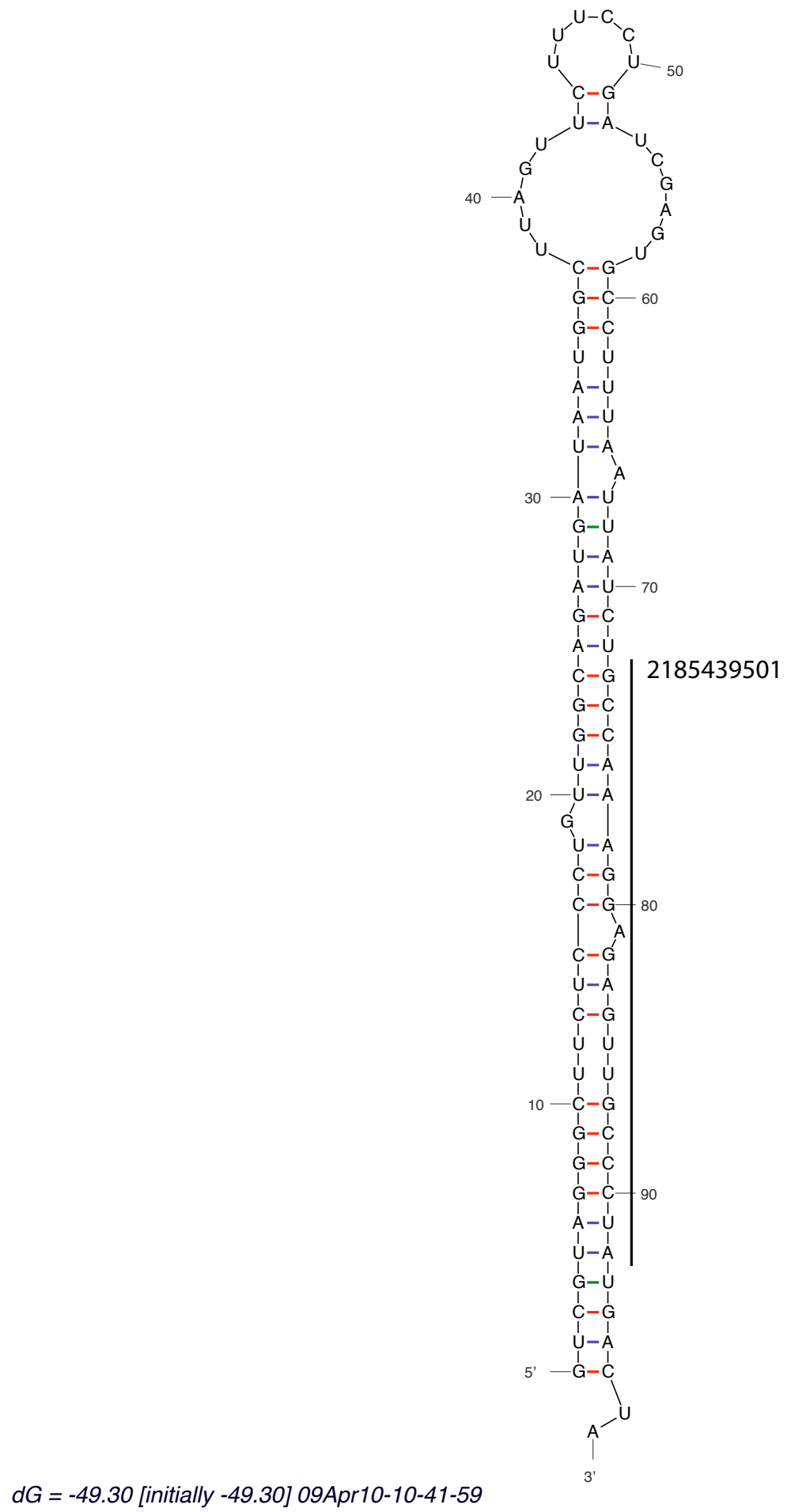


microRNA408
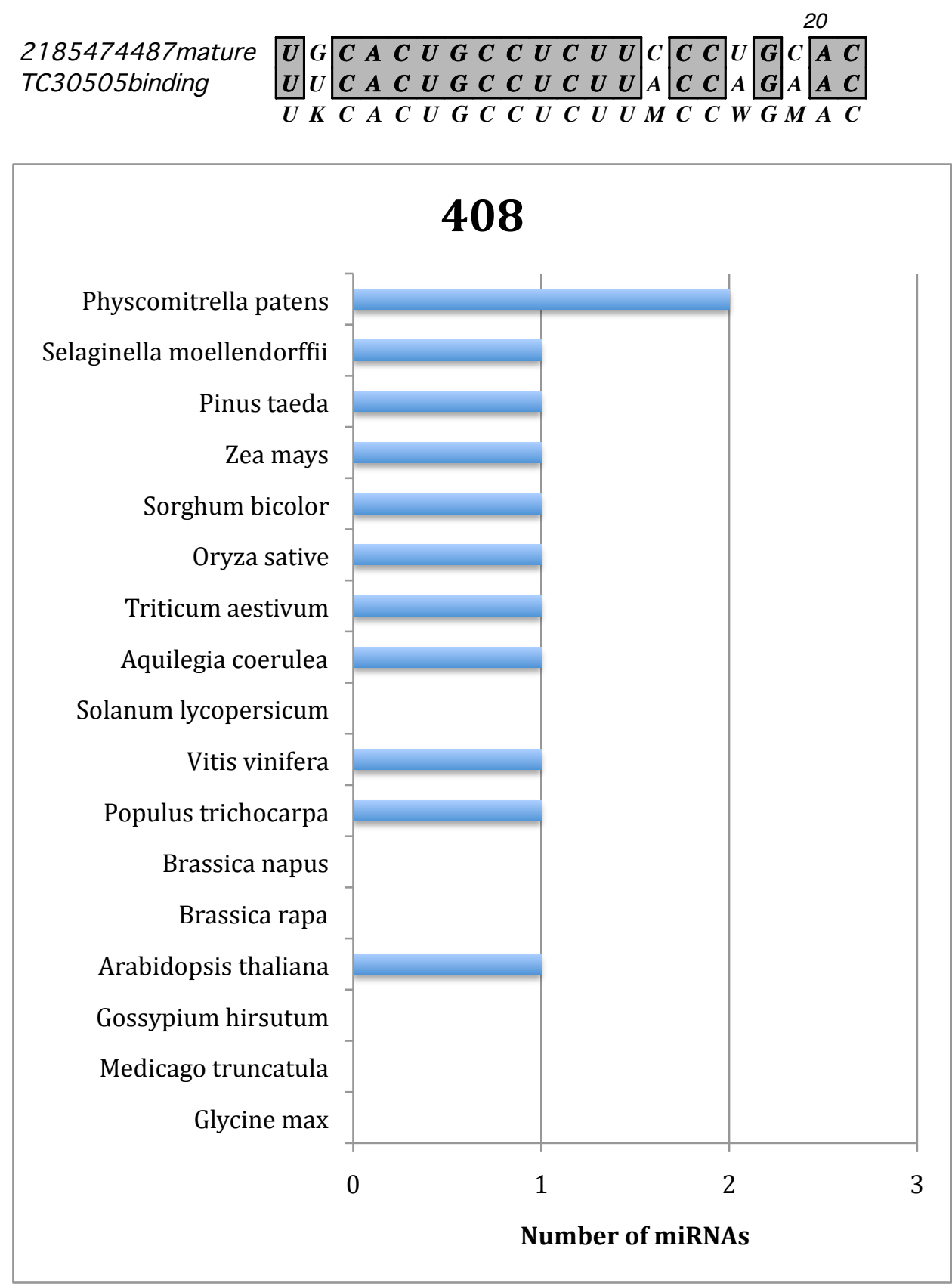
microRNA408

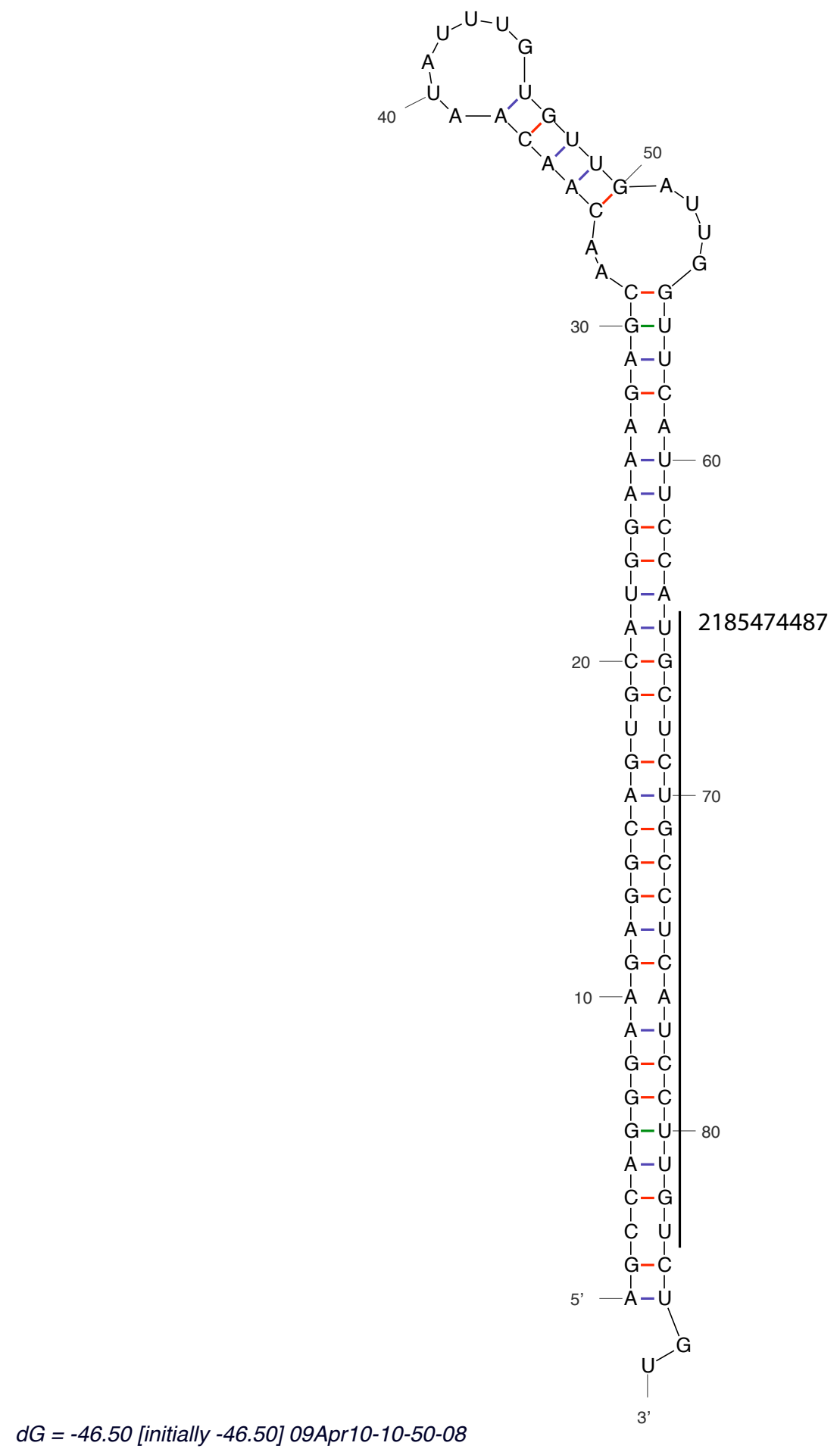


microRNA477

\begin{tabular}{|c|c|}
\hline & 20 \\
\hline 185809227 mature & 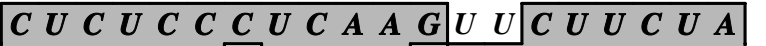 \\
\hline 2185853634 mature & 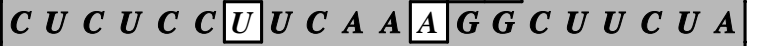 \\
\hline 2185874405 mature & 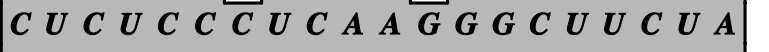 \\
\hline 2185477245 mature & 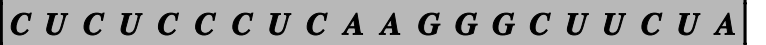 \\
\hline 2185552337 mature & 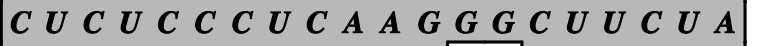 \\
\hline 2185755300 mature & 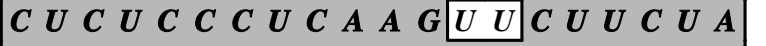 \\
\hline 2185768332 mature & 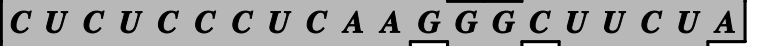 \\
\hline O303binding & 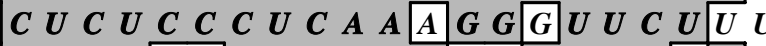 \\
\hline TC24529binding & 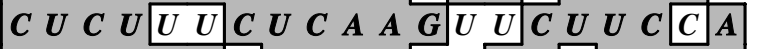 \\
\hline TC30732binding & 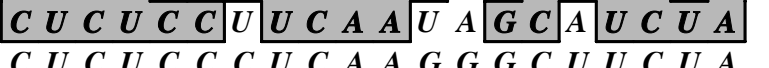 \\
\hline
\end{tabular}

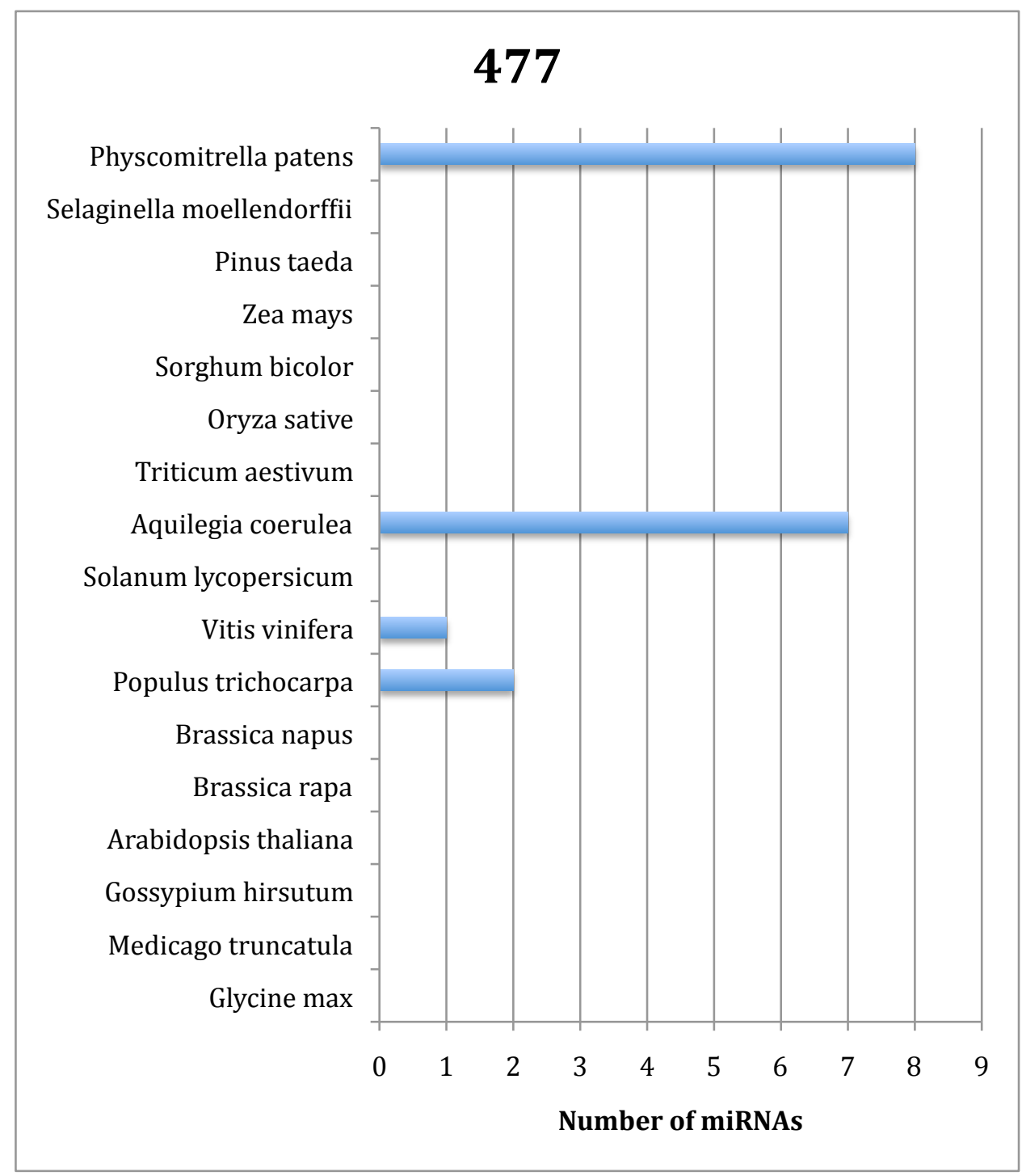

microRNA477 
microRNA477

Output of sir_graph (ब)
mfold 3.4

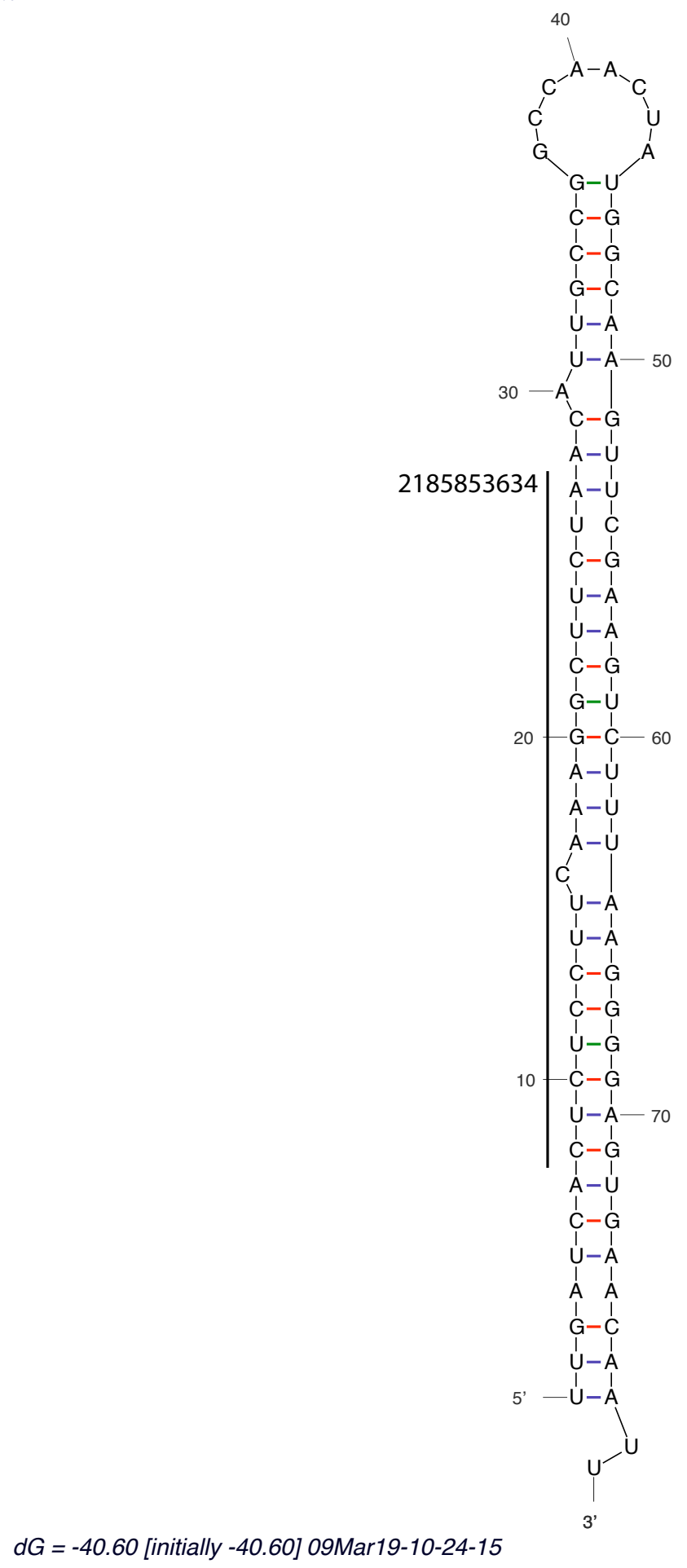

microRNA477 
microRNA477

Output of sir_graph (®)

$d G=-36.40$ [initially -36.40 ] 09Mar19-10-29-38

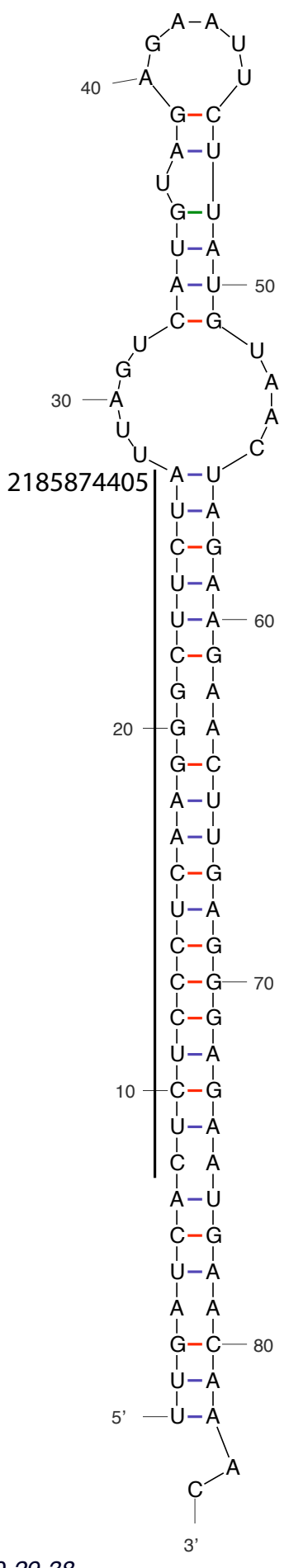

microRNA477 
microRNA477

Output of sir_graph (®)
mfold 3.4

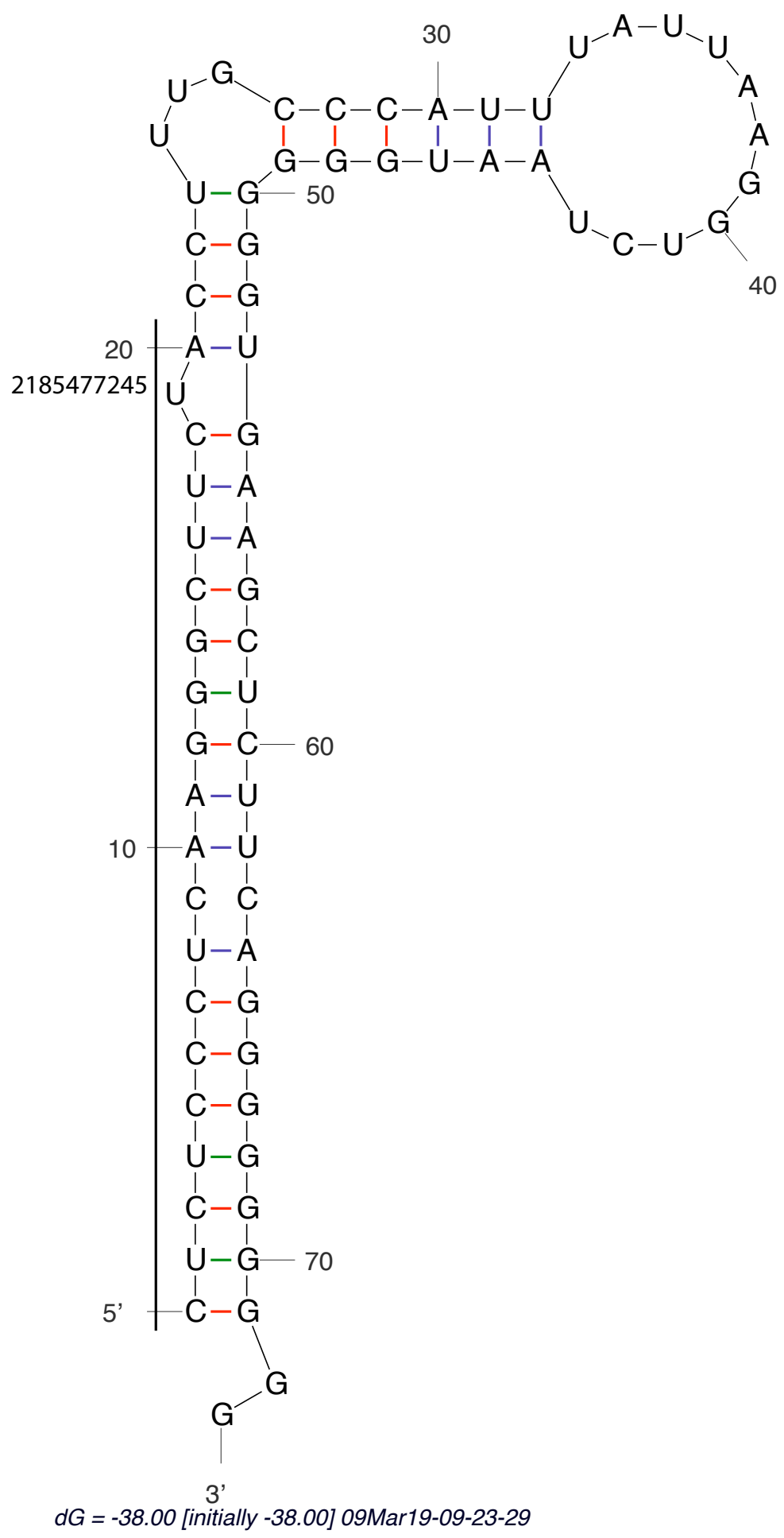

microRNA477 
microRNA477

Output of sir_graph (®)

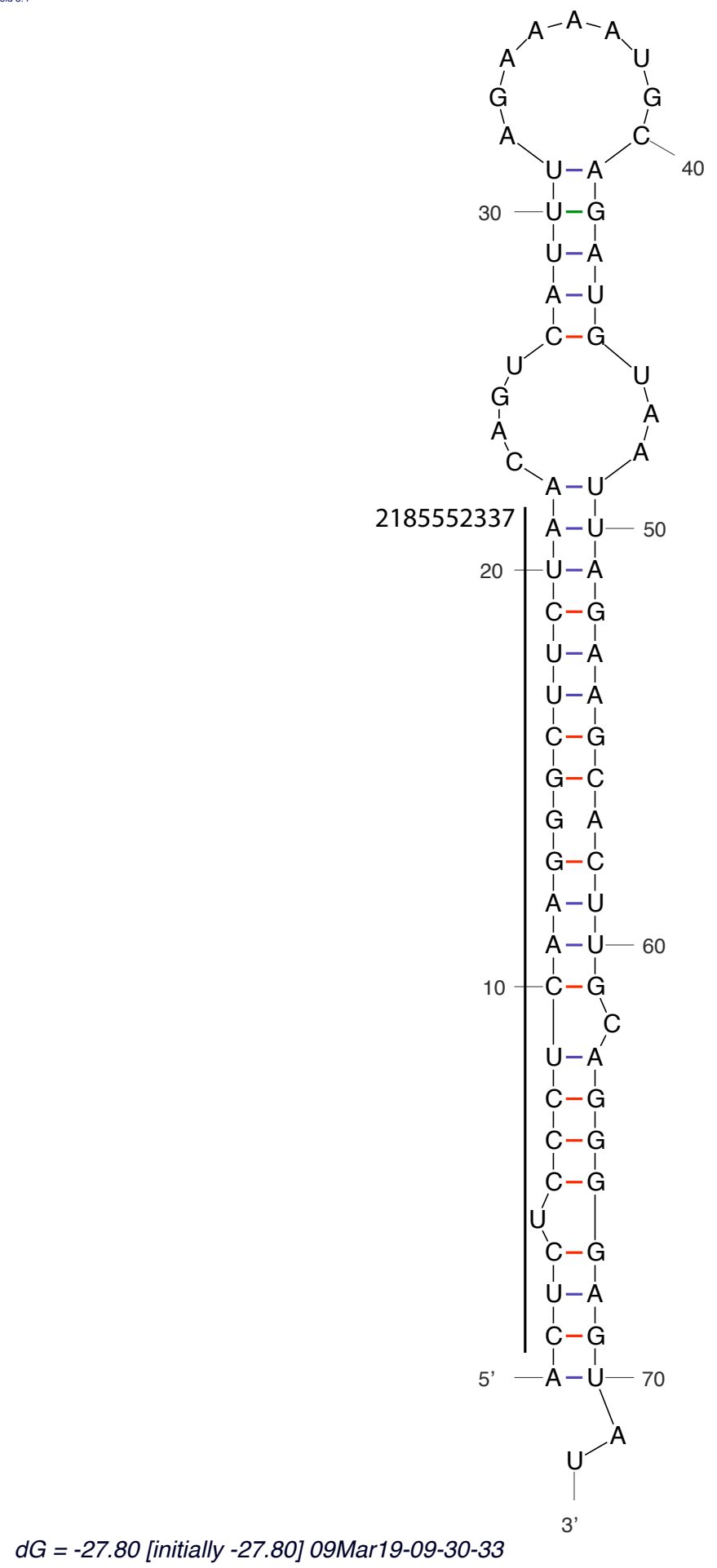

microRNA477 
microRNA477

Output of sir_graph (@)
mfold 3.4

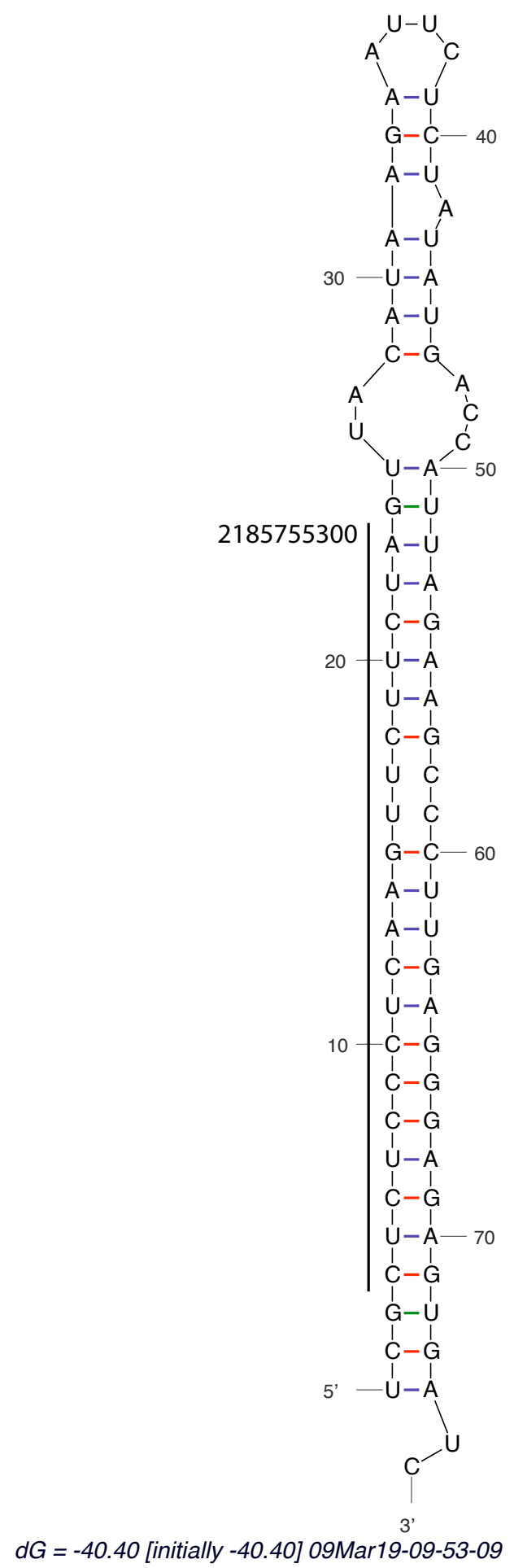

microRNA477 
microRNA477

Output of sir_graph (®)

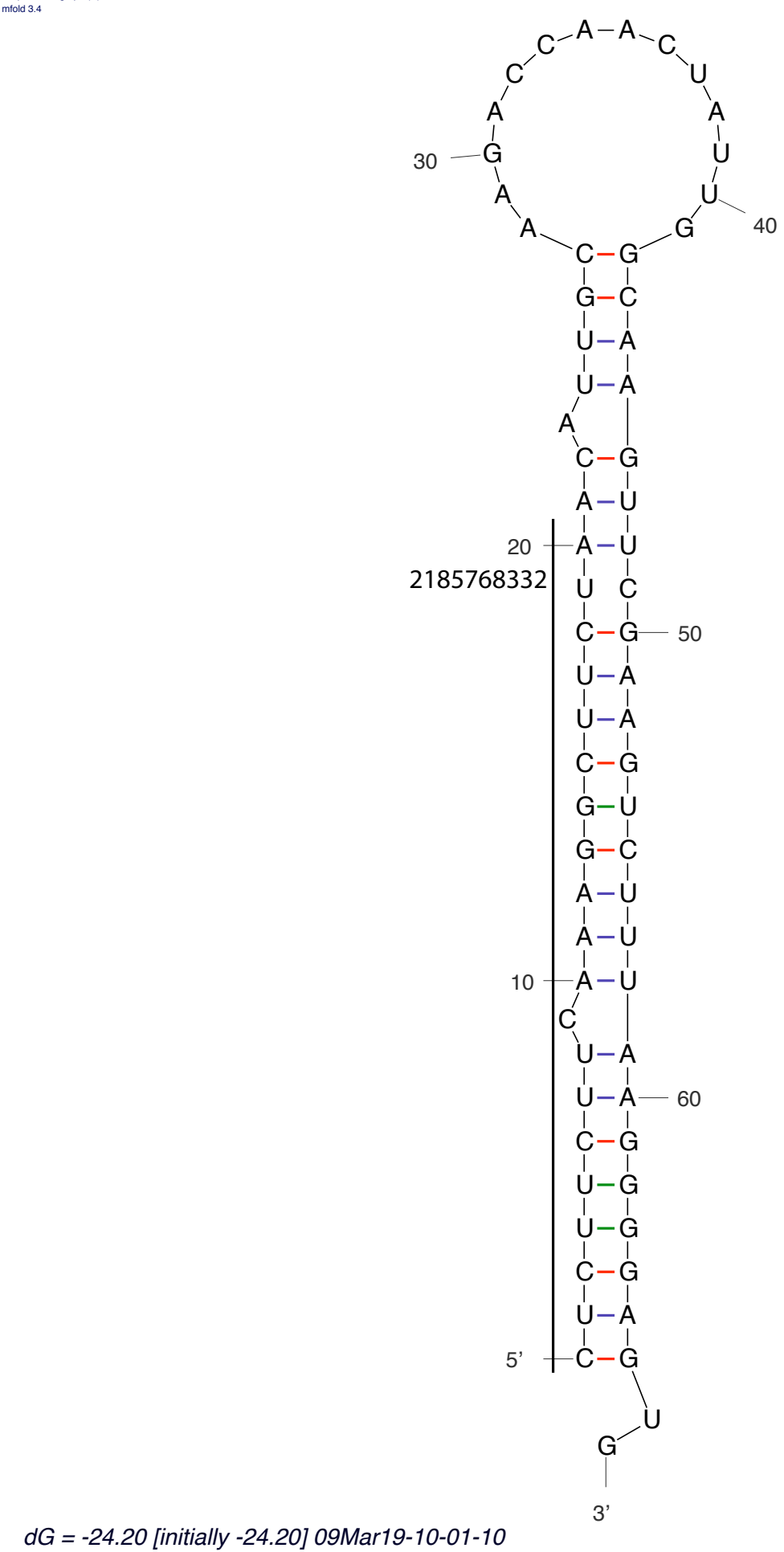

microRNA477 
microRNA477

Output of sir_graph (®)

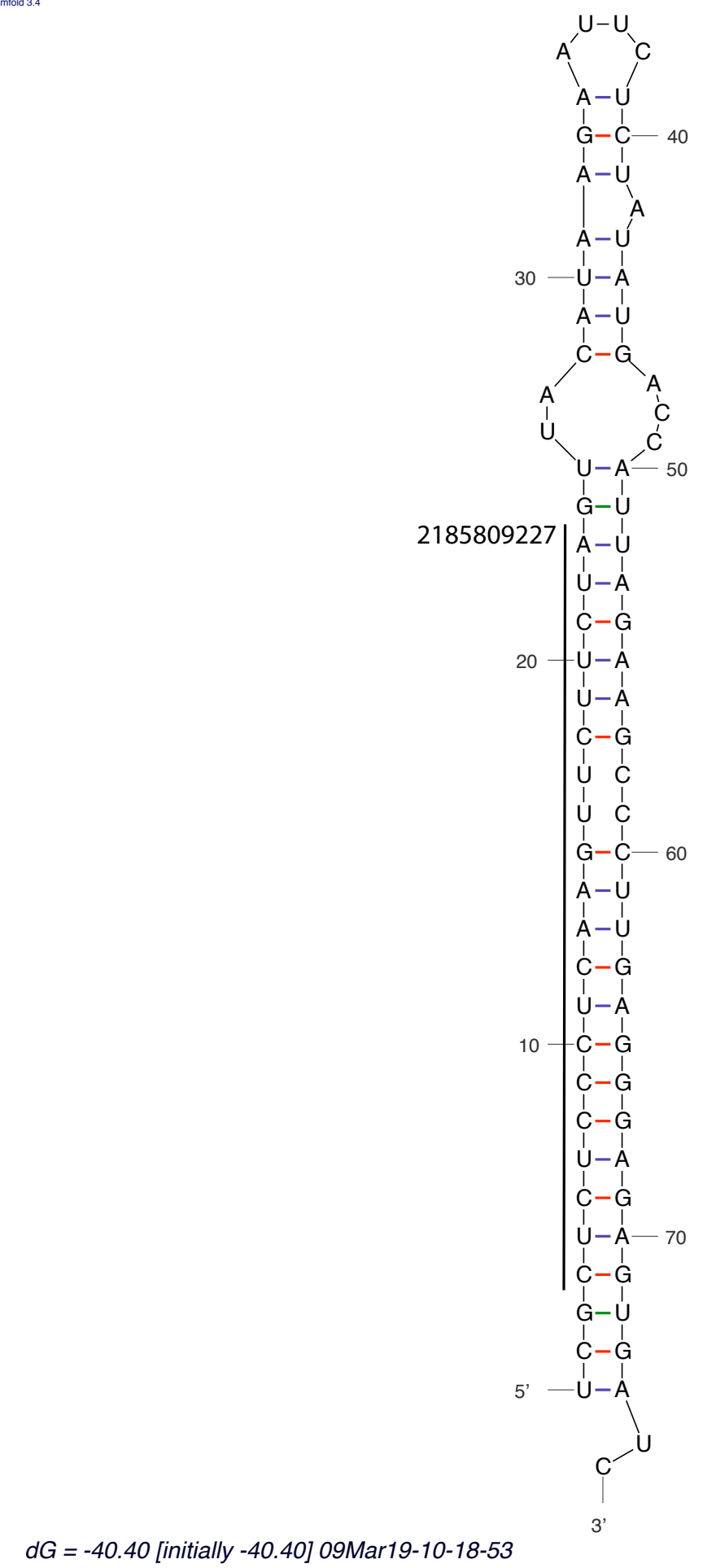

microRNA477 
microRNA482
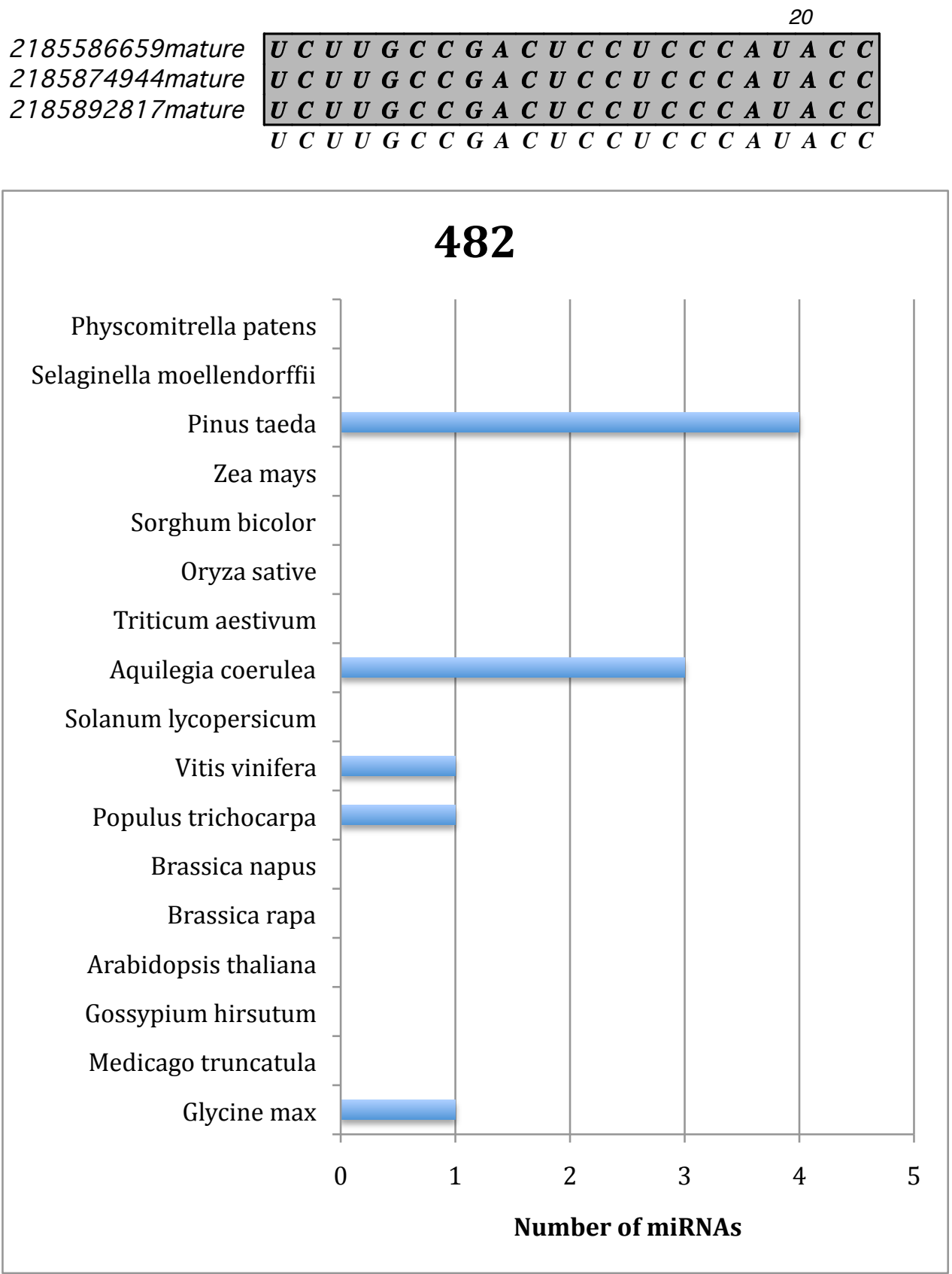
microRNA482

Output of sir_graph (ब)

Created Tue Apr 7 09:04:39 2009

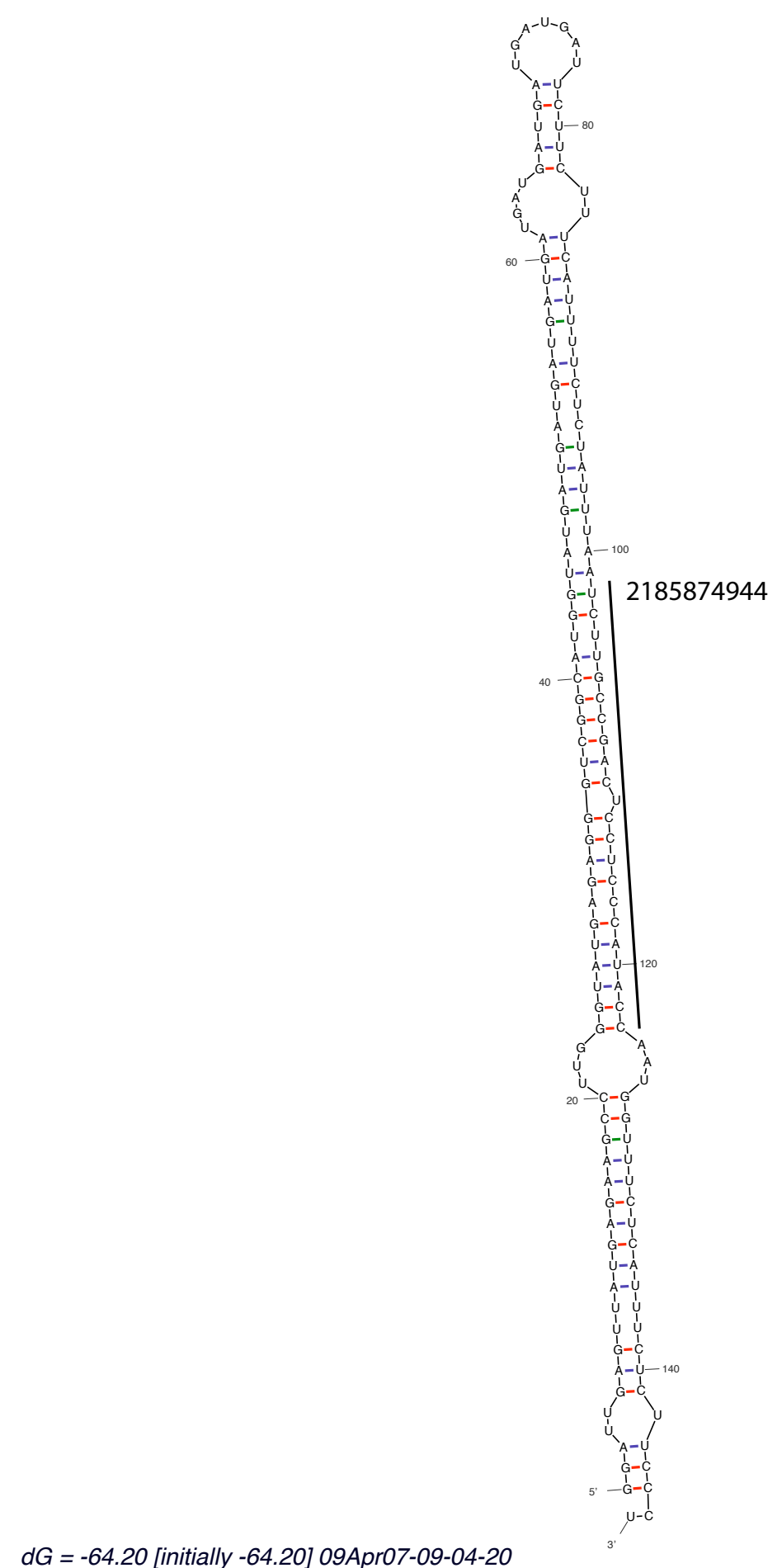

microRNA482 
microRNA482

Output of sir_graph (๑)

Created Tue Apr 708:39:40 2009

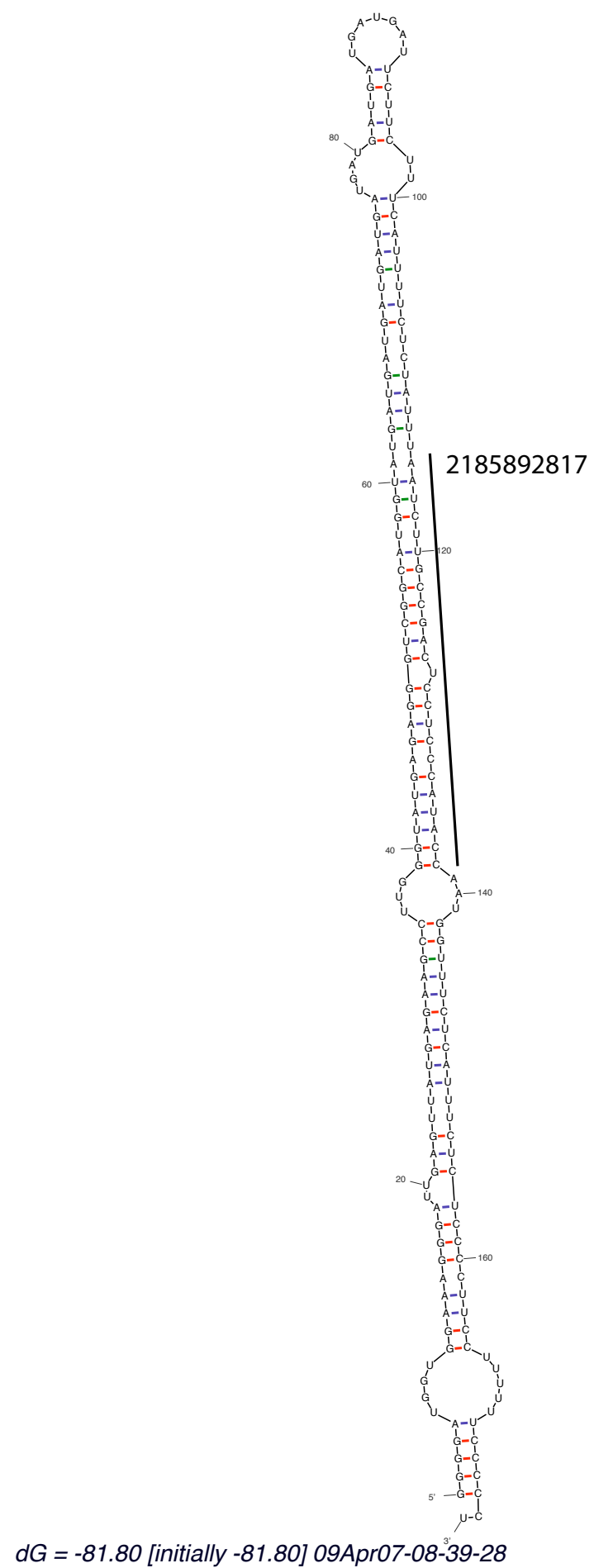

microRNA482 
microRNA482

Output of sir_graph (6)

Created Tue Apr 7 09:24:39 2009

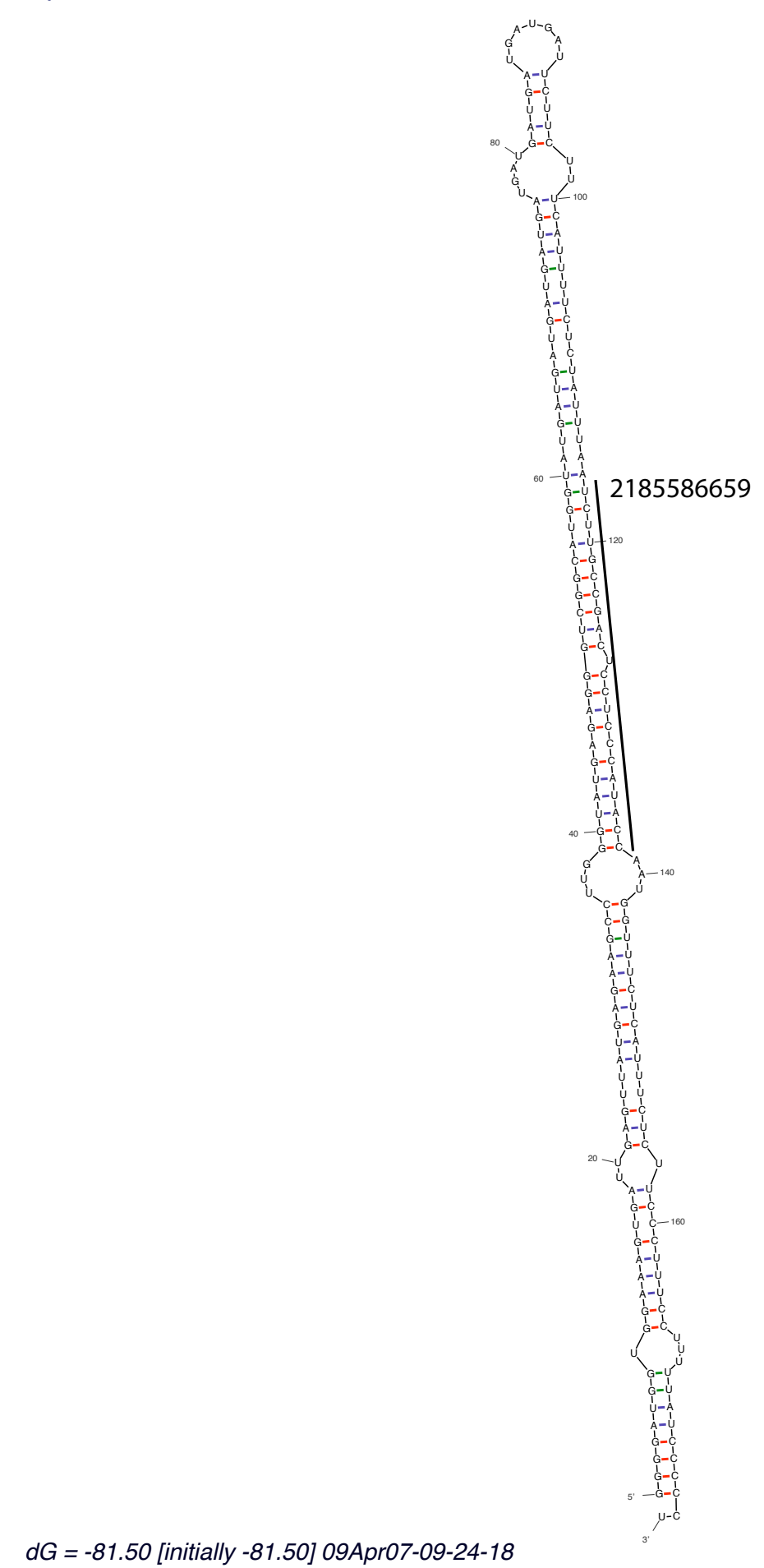

microRNA482 
microRNA529

2185620929mature
DR933604binding
DR949672binding
DR954092binding
DT745966binding
TC20719binding
TC21170binding
TC21707binding
TC21808binding
TC24142binding
TC24319binding
TC26823binding
TC29483binding
TC31356binding
TC31380binding
TC31827binding
TC32167binding
TC32968binding

\begin{tabular}{llllllllllllllllllllll}
\hline A & $G$ & $A$ & $A$ & $G$ & $A$ & $G$ & $A$ & $G$ & $A$ & $G$ & $A$ & $G$ & $C$ & $A$ & $C$ & $A$ & $A$ & $C$ & $C$ & $C$ & $C$
\end{tabular}

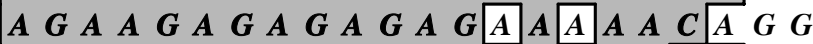
$\begin{array}{llllllllllllllllllllll}A & G & A & A & G & A & G & A & G & A & G & A & G & C & A & C & A & A & U & C & A & A\end{array}$ \begin{tabular}{lllllllllllllllll|lllll}
$A$ & $G$ & $A$ & $A$ & $G$ & $A$ & $G$ & $A$ & $G$ & $A$ & $G$ & $A$ & $G$ & $C$ & $A$ & $C$ & $G$ & $A$ & $C$ & $C$ & $A$ & $A$
\end{tabular}

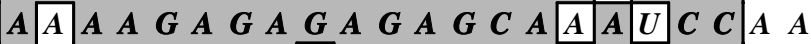
$\begin{array}{llllllllllllllllllllll}A & U & A & A & G & A & G & A & C & A & G & A & G & C & A & C & A & A & C & C & C & C\end{array}$ $\begin{array}{llllllllllllllllllllll}A & G & A & A & G & A & G & A & G & A & G & A & G & C & A & C & U & A & C & U & A & A\end{array}$ \begin{tabular}{lllllllllllllllll|l|lll}
$A$ & $G$ & $A$ & $A$ & $G$ & $A$ & $G$ & $A$ & $G$ & $A$ & $G$ & $A$ & $G$ & $C$ & $A$ & $C$ & $A$ & $G$ & $C$ & $U$ & $G$
\end{tabular}

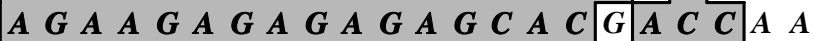

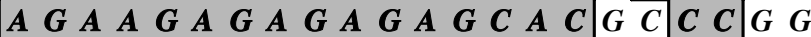

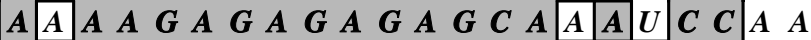

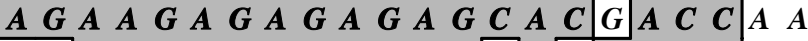
\begin{tabular}{lllllllllllllll|llllll}
$U$ & $\boldsymbol{A}$ & $\boldsymbol{A}$ & $\boldsymbol{G}$ & $\boldsymbol{A}$ & $\boldsymbol{G}$ & $\boldsymbol{A}$ & $\boldsymbol{G}$ & $\boldsymbol{A}$ & $\boldsymbol{G}$ & $\boldsymbol{A}$ & $\boldsymbol{G}$ & $\boldsymbol{A}$ & $\boldsymbol{A}$ & $\boldsymbol{A}$ & $\boldsymbol{A}$ & $\boldsymbol{A}$ & $\boldsymbol{C}$ & $\boldsymbol{C}$ & $\boldsymbol{C}$ & $\boldsymbol{C}$
\end{tabular} $\begin{array}{llllllllllllllllllllll}A & G & A & A & G & A & G & A & G & A & G & A & G & C & A & U & A & U & A & G & U & U\end{array}$ $\begin{array}{llllllllllllllllllllll}A & G & A & A & G & A & G & A & G & A & G & A & G & C & A & C & G & C & C & C & G & G\end{array}$ $\begin{array}{llllllllllllllllllllll}A & G & A & A & G & A & G & A & G & A & G & A & G & C & A & U & U & C & U & C & G & G\end{array}$

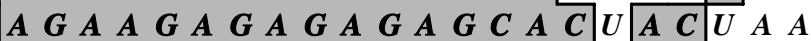
\begin{tabular}{lllllllllllll|llllllllll}
$A$ & $G$ & $A$ & $A$ & $G$ & $A$ & $G$ & $A$ & $G$ & $A$ & $G$ & $A$ & $A$ & $C$ & $G$ & $A$ & $A$ & $A$ & $C$ & $C$ & $C$ & $C$
\end{tabular} $\begin{array}{llllllllllllllllllllll}A & G & A & A & G & A & G & A & G & A & G & A & G & C & A & C & A & A & C & C & R\end{array}$

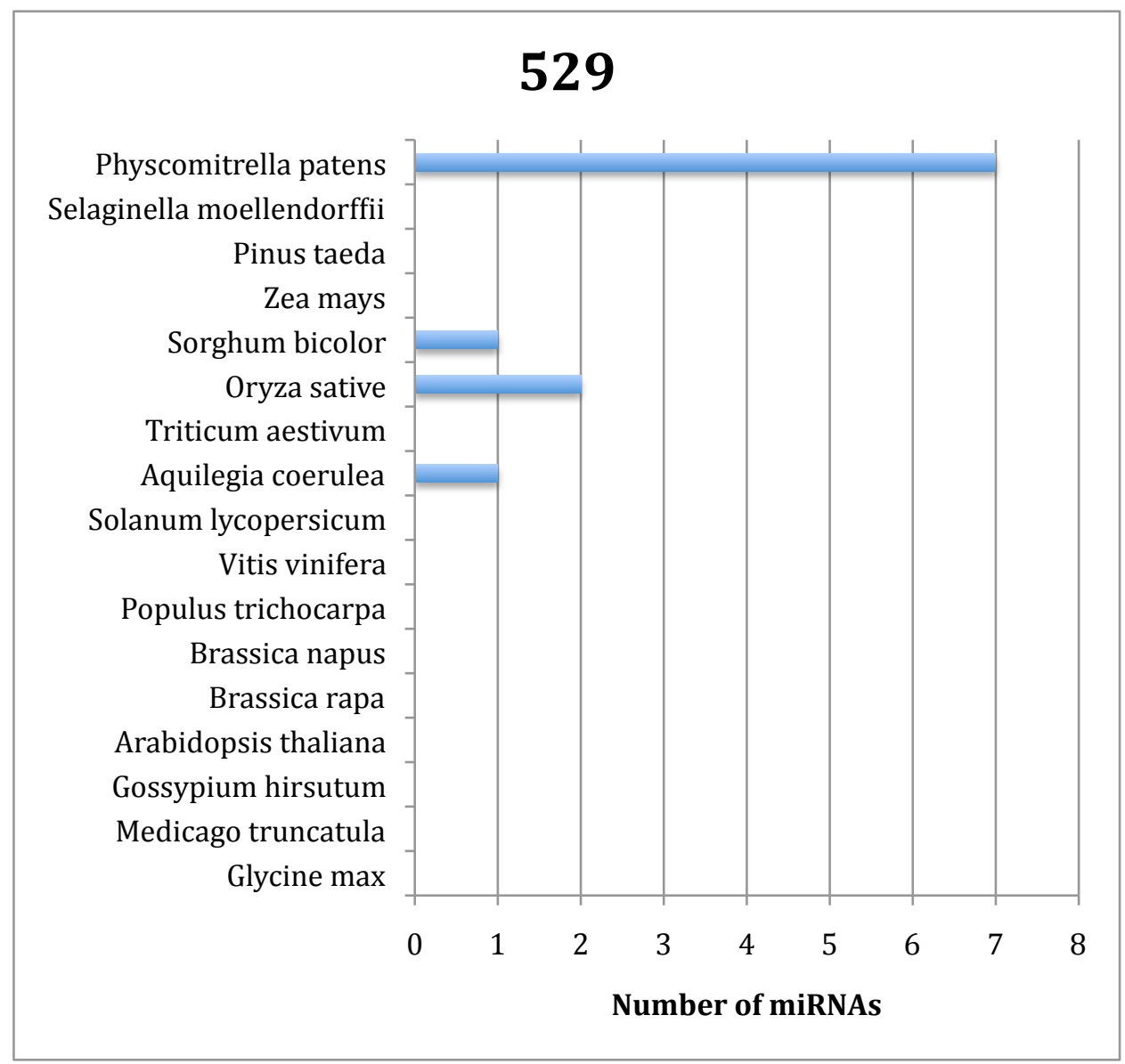

microRNA529 
microRNA529

Output of sir_graph (@)
mfold 3.4

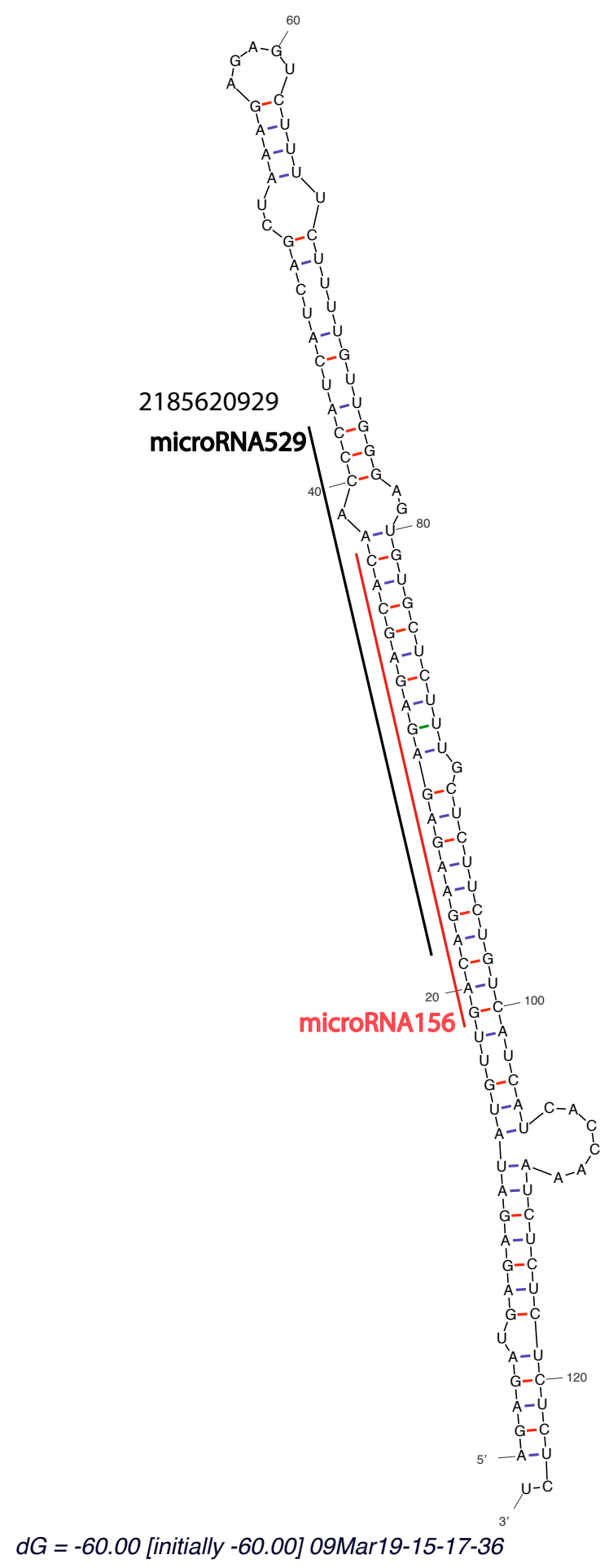

microRNA529 
microRNA530
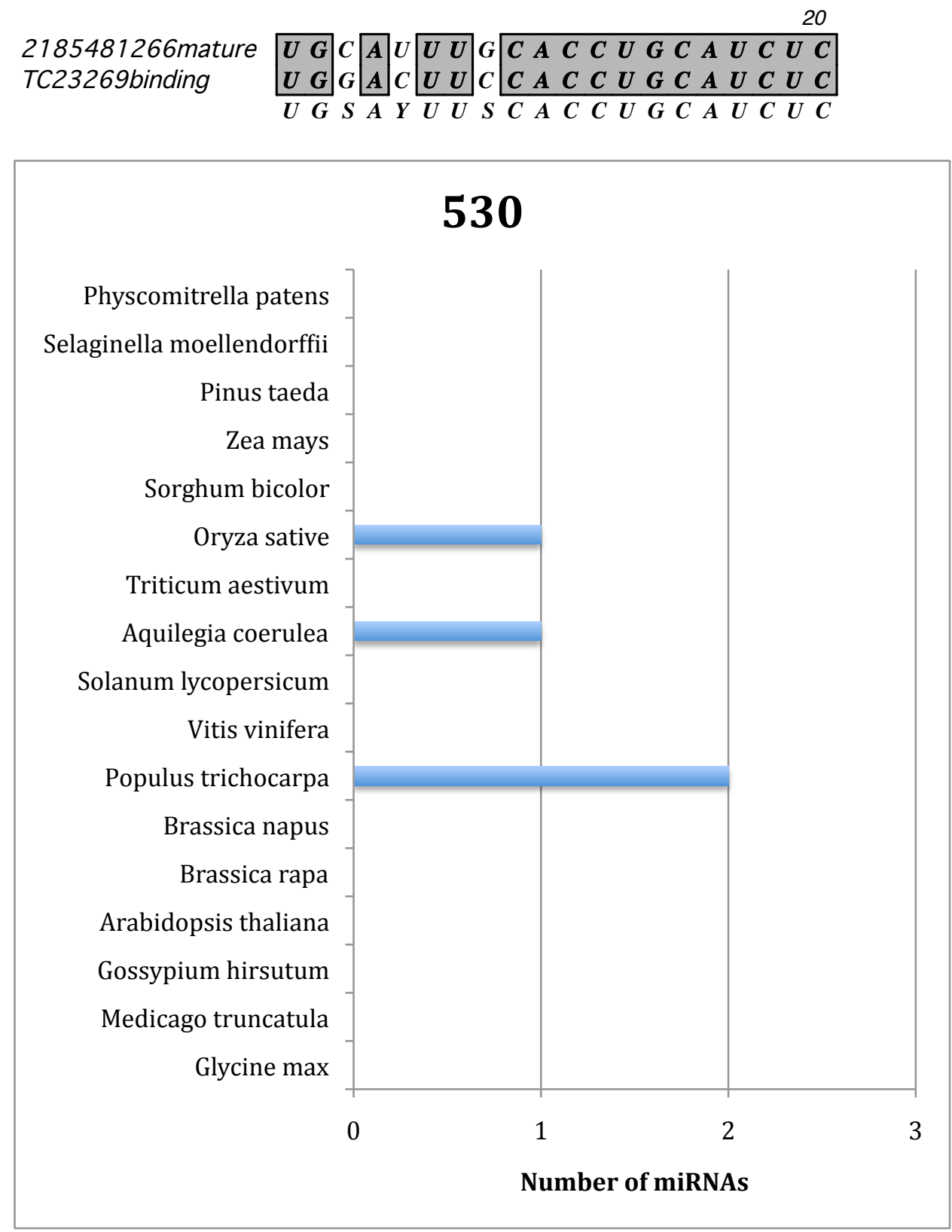
microRNA530

Output of sir_graph (๑)

Created Mon Mar 30 12:55:21 2009

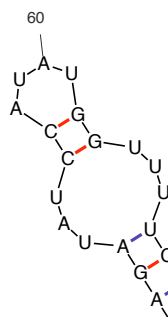

$d G=-62.80$ [initially -64.40 ] 09Mar30-12-55-11

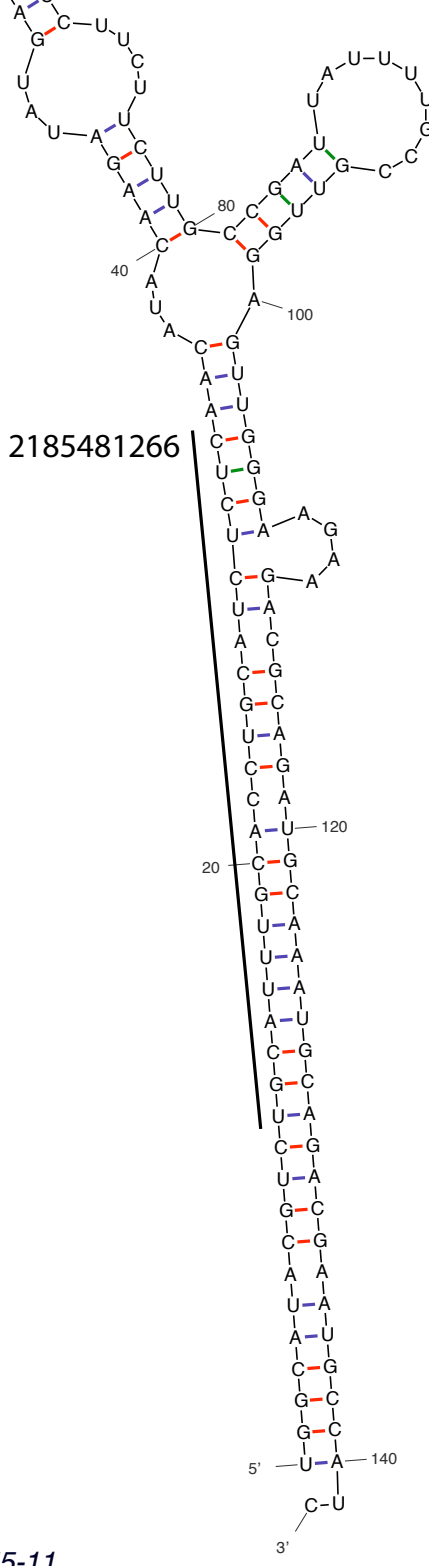

microRNA530 
microRNA535

20

$2185616263 m a t u r e \quad$\begin{tabular}{|llllllllllllllllllllll}
\hline & $\boldsymbol{G}$ & $\boldsymbol{A}$ & $\boldsymbol{C}$ & $\boldsymbol{A}$ & $\boldsymbol{A}$ & $\boldsymbol{C}$ & $\boldsymbol{G}$ & $\boldsymbol{A}$ & $\boldsymbol{G}$ & $\boldsymbol{A}$ & $\boldsymbol{G}$ & $\boldsymbol{A}$ & $\boldsymbol{G}$ & $\boldsymbol{A}$ & $\boldsymbol{G}$ & $\boldsymbol{C}$ & $\boldsymbol{A}$ & $\boldsymbol{C}$ & $\boldsymbol{G}$ & $\boldsymbol{C}$ & $\boldsymbol{G}$ \\
\hline
\end{tabular}

\begin{tabular}{lllllllllllllllllll}
\hline & $A$ & $C$ & $A$ & $A$ & $C$ & $G$ & $A$ & $G$ & $A$ & $G$ & $A$ & $G$ & $A$ & $G$ & $C$ & $A$ & $C$ & $G$
\end{tabular}

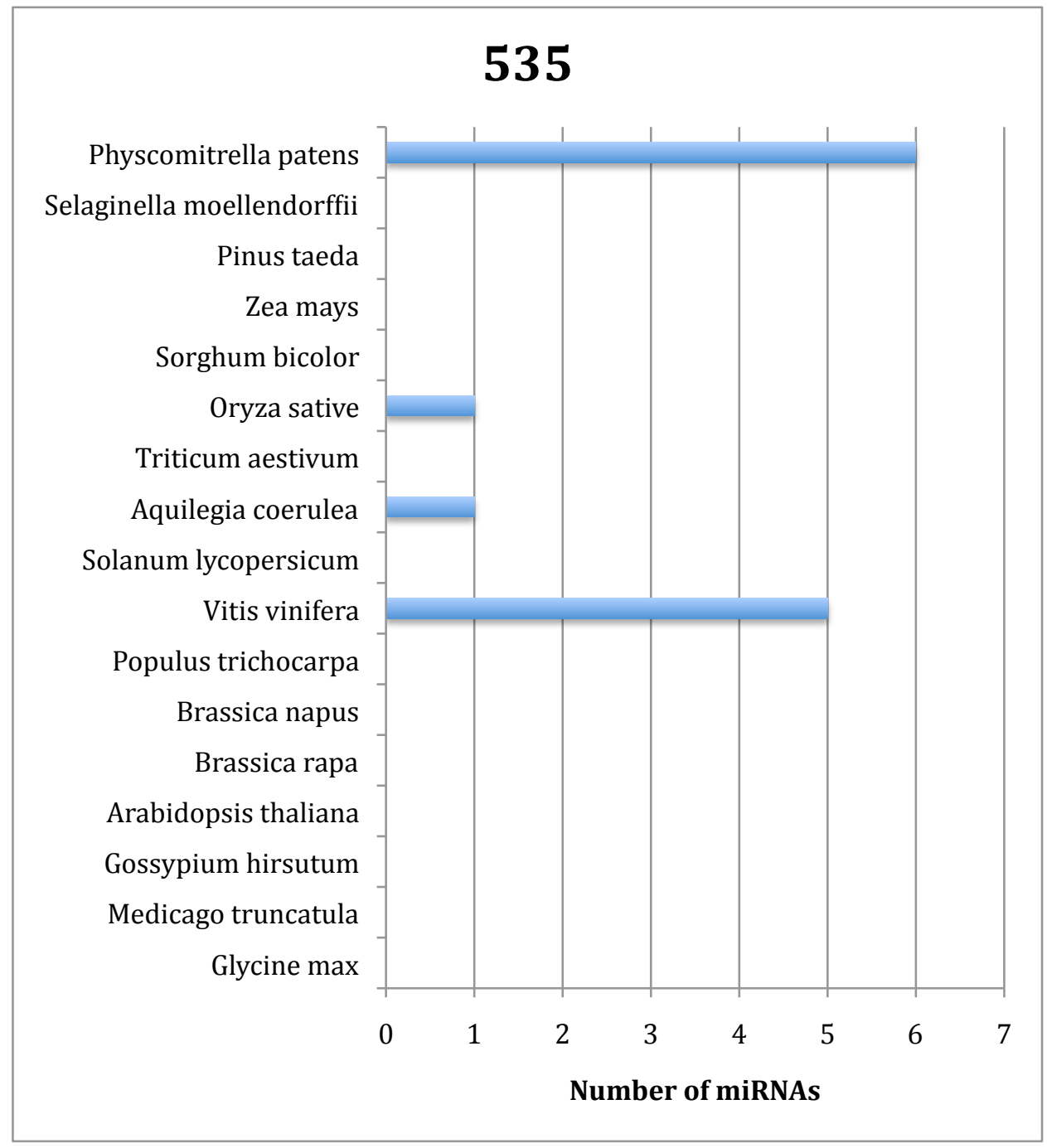


microRNA535

Output of sir_graph (®)

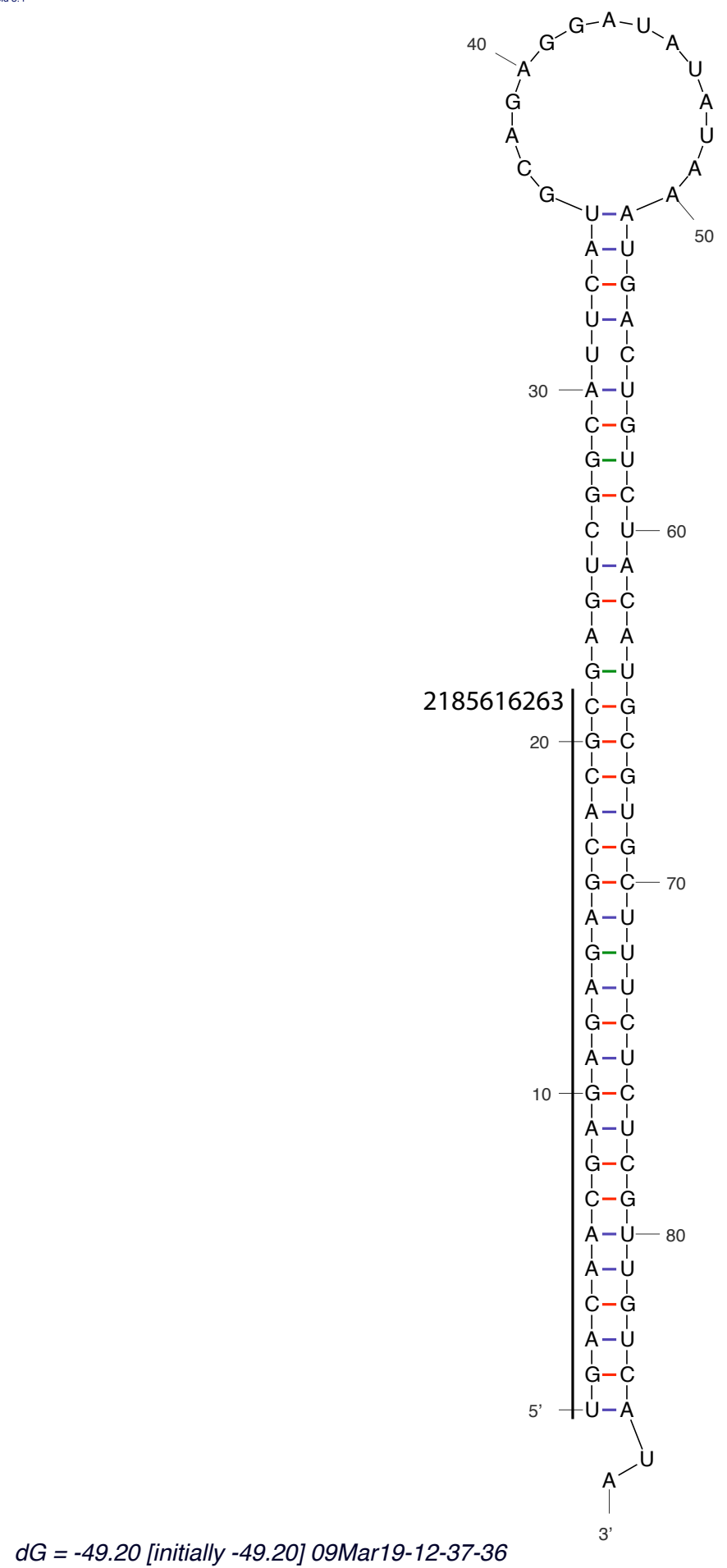

microRNA535 
microRNA783

\begin{tabular}{|c|c|}
\hline EST & 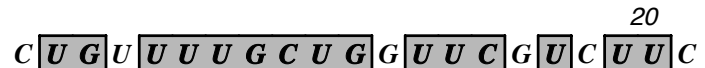 \\
\hline TC27593binding & 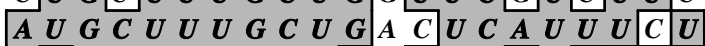 \\
\hline TC27947binding & 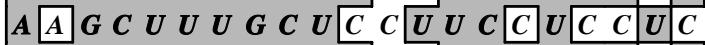 \\
\hline TC32408binding & 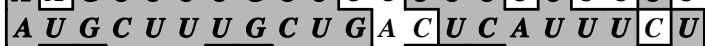 \\
\hline TC33228binding & 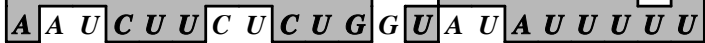 \\
\hline
\end{tabular}

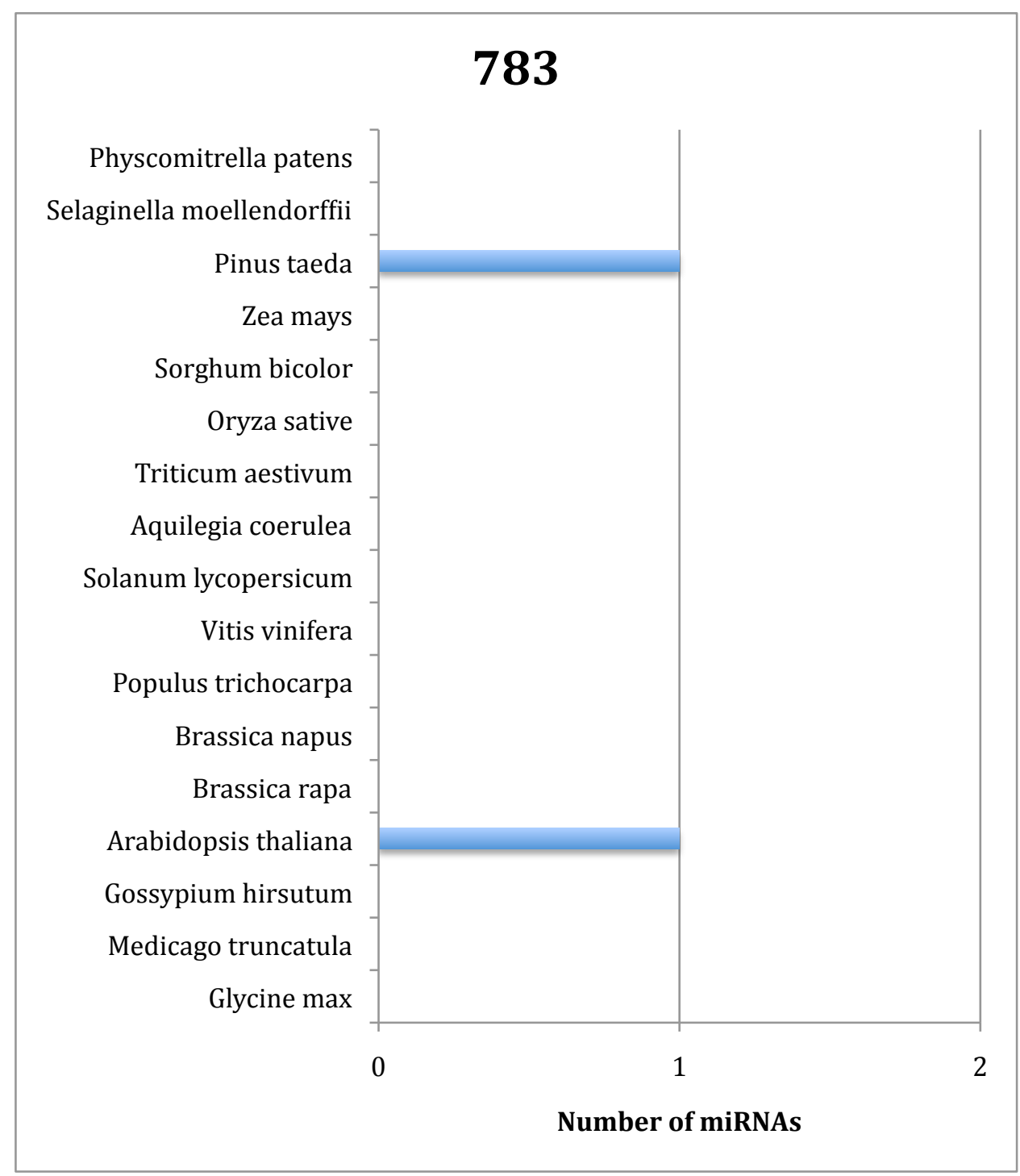

